\title{
Arylation, Vinylation, and Alkynylation of Electron-Deficient (Hetero)arenes Using Iodonium Salts
}

\author{
Chuan Liu and Qiu Wang* \\ Department of Chemistry, Duke University, Durham, NC 27708 \\ Email: qiu.wang@duke.edu
}

\section{SUPPORTING INFORMATION}

\section{Table of Contents}

$\begin{array}{ll}\text { I. General procedures } & \text { S2 }\end{array}$

$\begin{array}{ll}\text { II. Materials and instrumentation } & \text { S2 }\end{array}$

III. Experimental procedure and compound characterization for Scheme 2-4 S3-S16

IV. Synthesis and characterization of 3ca, 3cd, 3ce, and 3aq (Scheme 5). S17-S19

V. Experiment for the Synthesis of Compound 5 (Scheme 6). S19-S21

$\begin{array}{ll}\text { VI. References } & \text { S22-S23 }\end{array}$

$\begin{array}{ll}\text { VII. NMR spectra } & \text { S24-S97 }\end{array}$ 


\section{General Procedures.}

Glassware and stir bars were dried in an oven at $140{ }^{\circ} \mathrm{C}$ for at least $12 \mathrm{~h}$ and then cooled in a desiccator cabinet over Drierite prior to use. Optimization and substrate screens were performed in 8-mL microwave vials. Vials were fitted with crimp top septa under a positive pressure of nitrogen that had been passed through a column $(5 \times 20 \mathrm{~cm})$ of Drierite, unless otherwise noted. All other reactions were performed in round-bottom flasks sealed with rubber septa. Plastic syringes or glass pipets were used to transfer liquid reagents. Reactions were stirred magnetically using Teflon-coated, magnetic stir bars. Analytical thin-layer chromatography (TLC) was performed using aluminum plates pre-coated with $0.25 \mathrm{~mm}$ of 230-400 mesh silica gel impregnated with a fluorescent indicator $(254 \mathrm{~nm})$. TLC plates were visualized by exposure to ultraviolet light and/or exposure to $\mathrm{KMnO}_{4}$ stain. Organic solutions were concentrated under reduced pressure using a rotary evaporator. Flash-column chromatography was performed on silica gel (60 $\AA$, standard grade) or with pre-packed FLASH silica gel columns.

\section{Materials and Instrumentation.}

Nuclear magnetic resonance spectra were recorded at ambient temperature (unless otherwise stated) on $400 \mathrm{MHz}$ or $500 \mathrm{MHz}$ spectrometers. All values for proton chemical shifts are reported in parts per million $(\delta)$ and are referenced to the residual protium in $\mathrm{CDCl}_{3}(\delta$ 7.26). All values for carbon chemical shifts are reported in parts per million $(\delta)$ and are referenced to the carbon resonances in $\mathrm{CDCl}_{3}(\delta 77.0)$. NMR data are represented as follows: chemical shift, multiplicity $(\mathrm{s}=$ singlet, $\mathrm{d}=$ doublet, $\mathrm{t}=$ triplet, $\mathrm{q}=$ quartet, quin $=$ quintet, $\mathrm{m}=$ multiplet, $\mathrm{br}=$ broad $)$, coupling constant $(\mathrm{Hz})$, and integration. Infrared spectroscopic data are reported in wavenumbers $\left(\mathrm{cm}^{-1}\right)$. High-resolution mass spectra were obtained using a liquid chromatography-electrospray ionization and Time-

of-flight mass spectrometer. The diaryliodonium triflates, ${ }^{1}$ vinyl iodonium salts ${ }^{2}$ and phenylalynyl iodoium salt ${ }^{3}$ were prepared according to the reported procedures. Copper(I) trifluoromethanesulfonate toluene complex was purchased from Sigma Aldrich. 


\section{Experimental procedure and compound characterization for Scheme 2-4.}

\section{III-a. General procedure for arylation, vinylation and alkynylaton of electron-}

deficient (hetereo)arenes (Scheme 2-4).

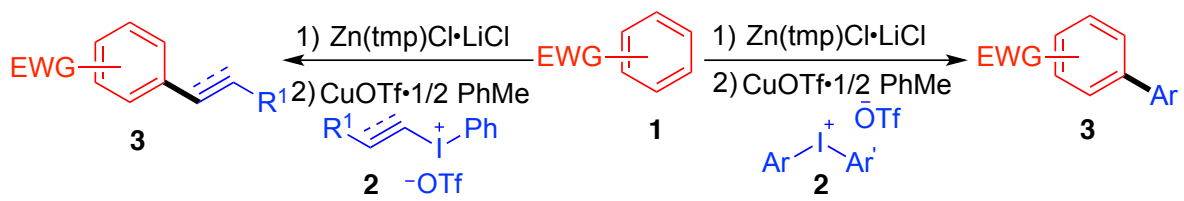

To an oven dried Schlenk tube was added compound 1 ( $0.3 \mathrm{mmol}, 1.0$ equiv) and the solution of $\mathrm{Zn}(\mathrm{tmp}) \mathrm{Cl} \cdot \mathrm{LiCl}(0.6 \mathrm{mmol}, 2.0$ equiv) in THF ( $3 \mathrm{~mL})$ under $\mathrm{N}_{2}$. The mixture was allowed to stir at $50{ }^{\circ} \mathrm{C}$ for $1 \mathrm{~h}$. To the reaction mixture, was added iodonium salt 2 (0.6 mmol, 2.0 equiv), CuOTf $\bullet 1 / 2 \mathrm{PhMe}(7.8 \mathrm{mg}, 0.03 \mathrm{mmol}, 10 \mathrm{~mol} \%$ ) and anhydrous THF (3.0 mL) under $\mathrm{N}_{2}$. The resulting mixture was allowed to stir at the noted temperature. Upon the complete consumption of $\mathbf{1}$ (monitored by TLC), the reaction was quenched by the addition of $\mathrm{MeOH}(1 \mathrm{~mL})$. Then the mixture was filtered though a plug of $\mathrm{Al}_{2} \mathrm{O}_{3}$ and washed with $\mathrm{Et}_{2} \mathrm{O}(5 \mathrm{~mL} \times 3)$ and $\mathrm{CH}_{2} \mathrm{Cl}_{2}(5 \mathrm{~mL} \times 3)$. The combined organic layers were concentrated in vacuo. Purification of the crude mixture by silica column chromatography afforded product 3 .

\section{III-b. Characterization of biaryl compounds 3aa-3an (Scheme 2).}

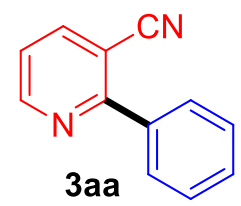

2-Phenylnicotinonitrile (3aa). Purification by silica column chromatography (10\% EtOAc in hexanes) afforded 3aa as a light yellow solid ( $44.5 \mathrm{mg}, 82 \%$ yield). $\mathrm{R}_{f}=0.28$ (17\% EtOAc in hexanes); ${ }^{1} \mathrm{H}$ NMR (400 MHz, $\left.\mathrm{CDCl}_{3}\right) \delta 8.88$ (dd, $\left.J=4.8,2.0 \mathrm{~Hz}, 1 \mathrm{H}\right)$, 8.08 (dd, $J=8.0,2.0 \mathrm{~Hz}, 1 \mathrm{H}), 7.94-7.91$ (m, 2H), 7.56-7.52 (m, 3H), 7.38 (dd, $J=8.0$, $4.8 \mathrm{~Hz}, 1 \mathrm{H})$. Spectroscopic data was identical to that reported previously. ${ }^{4}$ 


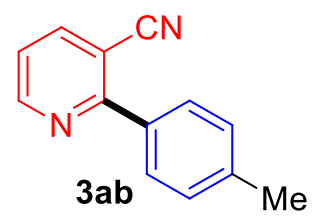

2-(p-Tolyl)nicotinonitrile (3ab). Purification by silica column chromatography $(5 \% \rightarrow 10 \%$ EtOAc in hexanes) afforded $\mathbf{3 a b}$ as a white solid (40.9 mg, 70\% yield). $\mathrm{R}_{f}=0.29$ (17\% EtOAc in hexanes); ${ }^{1} \mathrm{H}$ NMR (400 MHz, $\left.\mathrm{CDCl}_{3}\right) \delta 8.85(\mathrm{dd}, J=4.8,2.0 \mathrm{~Hz}, 1 \mathrm{H}), 8.05$ $(\mathrm{dd}, J=8.0,2.0 \mathrm{~Hz}, 1 \mathrm{H}), 7.85-7.83(\mathrm{~m}, 2 \mathrm{H}), 7.35-7.32(\mathrm{~m}, 3 \mathrm{H}), 2.43(\mathrm{~s}, 3 \mathrm{H})$. Spectroscopic data was identical to that reported previously. ${ }^{5}$

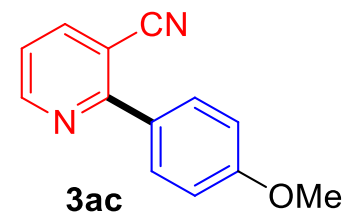

2-(4-Methoxyphenyl)nicotinonitrile (3ac). Purification by silica column chromatography $(5 \% \rightarrow 10 \%$ EtOAc in hexanes) afforded 3ac as a white solid (40.6 mg, $64 \%$ yield). $\mathrm{R}_{f}=0.25$ (17\% EtOAc in hexanes); ${ }^{1} \mathrm{H}$ NMR (400 $\left.\mathrm{MHz}, \mathrm{CDCl}_{3}\right) \delta 8.83$ (dd, $J=4.8,2.0 \mathrm{~Hz}, 1 \mathrm{H}), 8.03(\mathrm{dd}, J=8.0,2.0 \mathrm{~Hz}, 1 \mathrm{H}), 7.96-7.89(\mathrm{~m}, 2 \mathrm{H}), 7.30$ (dd, $J=8.0$, $4.8 \mathrm{~Hz}, 1 \mathrm{H}), 7.06-7.02(\mathrm{~m}, 2 \mathrm{H}), 3.87(\mathrm{~s}, 3 \mathrm{H})$; Spectroscopic data was identical to that reported previously. ${ }^{6}$<smiles>N#Cc1cccnc1-c1ccc(F)cc1</smiles>

2-(4-Fluorophenyl)nicotinonitrile (3ad). Purification by silica column chromatography $\left(5 \% \rightarrow 10 \%\right.$ EtOAc in hexanes) afforded $\mathbf{3 a d}$ as a white solid $(50.2 \mathrm{mg}, 84 \%$ yield $) . \mathrm{R}_{f}=$ 0.30 (17\% EtOAc in hexanes); ${ }^{1} \mathrm{H}$ NMR $\left(400 \mathrm{MHz}, \mathrm{CDCl}_{3}\right) \delta 8.86(\mathrm{dd}, J=4.8,2.0 \mathrm{~Hz}$, $1 \mathrm{H}), 8.07$ (dd, $J=8.0,2.0 \mathrm{~Hz}, 1 \mathrm{H}), 7.96-7.93(\mathrm{~m}, 2 \mathrm{H}), 7.38(\mathrm{dd}, J=8.0,4.8 \mathrm{~Hz}, 1 \mathrm{H})$, 7.24-7.19 (m, 2H); ${ }^{19} \mathrm{~F}$ NMR (376 MHz, $\left.\mathrm{CDCl}_{3}\right) \delta-110.24(\mathrm{dq}, J=8.5,5.3 \mathrm{~Hz})$; Spectroscopic data was identical to that reported previously. ${ }^{7}$ 


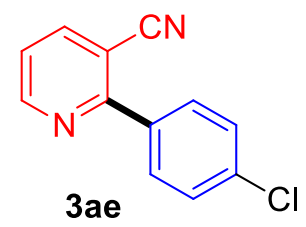

2-(4-chlorophenyl)nicotinonitrile (3ae). Purification by silica column chromatography $\left(5 \% \rightarrow 10 \%\right.$ EtOAc in hexanes) afforded 3ae as a white solid ( $40.3 \mathrm{mg}, 63 \%$ yield). $\mathrm{R}_{f}=$ 0.29 (17\% EtOAc in hexanes); ${ }^{1} \mathrm{H}$ NMR $\left(400 \mathrm{MHz}, \mathrm{CDCl}_{3}\right) \delta 8.87$ (dd, $J=4.8,2.0 \mathrm{~Hz}$, $1 \mathrm{H}), 8.08$ (dd, $J=8.0,2.0 \mathrm{~Hz}, 1 \mathrm{H}), 7.91-7.88$ (m, 2H), 7.52-7.49 (m, 2H), 7.40 (dd, $J=$ 8.0, $4.8 \mathrm{~Hz}, 1 \mathrm{H}) ;{ }^{13} \mathrm{C} \mathrm{NMR}\left(100 \mathrm{MHz}, \mathrm{CDCl}_{3}\right) \delta 159.6,152.7,141.8,136.5,135.4,130.2$, 129.0, 121.8, 117.5, 107.4. IR (film): $\mathrm{cm}^{-1} 2223,1595,1579,1549,1430,1399,1090$, 1013, 805, 770, 737; HRMS-ESI (m/z) calcd for $\mathrm{C}_{12} \mathrm{H}_{8} \mathrm{ClN}_{2}\left([\mathrm{M}+\mathrm{H}]^{+}\right)$: 215.0371. Found: 215.0368 .

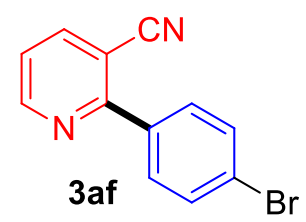

2-(4-Bromophenyl)nicotinonitrile (3af). Purification by silica column chromatography ( $5 \% \rightarrow 10 \%$ EtOAc in hexanes) afforded 3af as a white solid ( $47.9 \mathrm{mg}, 62 \%$ yield). $\mathrm{R}_{f}=$ 0.27 (17\% EtOAc in hexanes); ${ }^{1} \mathrm{H} \mathrm{NMR}\left(400 \mathrm{MHz}, \mathrm{CDCl}_{3}\right) \delta 8.87(\mathrm{dd}, J=4.8,2.0 \mathrm{~Hz}$, $1 \mathrm{H}), 8.08(\mathrm{dd}, J=8.0,2.0 \mathrm{~Hz}, 1 \mathrm{H}), 7.84-7.80(\mathrm{~m}, 2 \mathrm{H}), 7.8-7.65(\mathrm{~m}, 2 \mathrm{H}), 7.40(\mathrm{dd}, J=$ 8.0, $4.8 \mathrm{~Hz}, 1 \mathrm{H}) ;{ }^{13} \mathrm{C} \mathrm{NMR}\left(100 \mathrm{MHz}, \mathrm{CDCl}_{3}\right) \delta 159.8,152.7,141.9,135.9,131.9,130.4$, 125.0, 121.8, 117.4, 107.3. IR (film): $\mathrm{cm}^{-1}$ 2224, 1589, 1578, 1552, 1431, 1394, 1074, 1011, 806, 769, 724; HRMS-ESI (m/z) calcd for $\mathrm{C}_{12} \mathrm{H}_{8} \mathrm{BrN}_{2}\left([\mathrm{M}+\mathrm{H}]^{+}\right)$: 258.9865. Found: 258.9861.

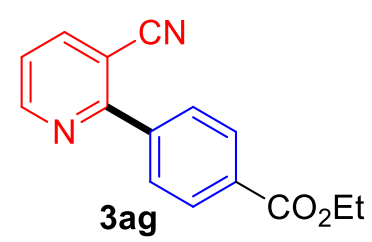

Ethyl 4-(3-cyanopyridin-2-yl)benzoate (3ag). Purification by silica column chromatography $(5 \% \rightarrow 10 \%$ EtOAc in hexanes) afforded 3ag as a white solid (53.6 mg, $71 \%$ yield). $\mathrm{R}_{f}=0.19$ (17\% EtOAc in hexanes); ${ }^{1} \mathrm{H}$ NMR (400 MHz, $\left.\mathrm{CDCl}_{3}\right) \delta 8.89$ (dd, 
$J=4.8,2.0 \mathrm{~Hz}, 1 \mathrm{H}), 8.20-8.18(\mathrm{~m}, 2 \mathrm{H}), 8.09$ (dd, $J=8.0,2.0 \mathrm{~Hz}, 1 \mathrm{H}), 8.00-7.98(\mathrm{~m}$, 2H), 7.42 (dd, $J=8.0,4.8 \mathrm{~Hz}, 1 \mathrm{H}), 4.41$ (q, $J=7.2 \mathrm{~Hz}, 2 \mathrm{H}), 1.41$ (t, $J=7.2 \mathrm{~Hz}, 3 \mathrm{H}$ ); Spectroscopic data was identical to that reported previously. ${ }^{8}$

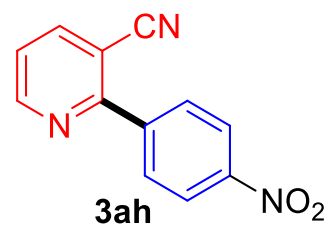

2-(4-Nitrophenyl)nicotinonitrile(3ah). Purification by silica column chromatography $(5 \% \rightarrow 10 \%$ EtOAc in hexanes) afforded 3ah as a light yellow solid (45.8 $\mathrm{mg}, 68 \%$ yield). $\mathrm{R}_{f}=0.21$ (17\% EtOAc in hexanes); ${ }^{1} \mathrm{H}$ NMR $\left(400 \mathrm{MHz}, \mathrm{CDCl}_{3}\right) \delta 8.94(\mathrm{~d}, J=4.8 \mathrm{~Hz}$, $1 \mathrm{H}), 8.39$ (dd, $J=8.8,1.2 \mathrm{~Hz}, 2 \mathrm{H}), 8.16-8.11$ (m, 3H), 7.50 (dd, $J=7.6,4.8 \mathrm{~Hz}, 1 \mathrm{H}$ ); ${ }^{13} \mathrm{C}$ NMR $\left(100 \mathrm{MHz}, \mathrm{CDCl}_{3}\right) \delta 158.5,152.9,148.7,142.8,141.9,130.0,123.8,122.8$, 116.9, 108.0. IR (film): $\mathrm{cm}^{-1} 3479,1512,1434,1354,1247,1227,1176,1030,857,805$, 780, 740, 723; HRMS-ESI (m/z) calcd for $\mathrm{C}_{12} \mathrm{H}_{8} \mathrm{ClN}_{3} \mathrm{O}_{2}\left([\mathrm{M}+\mathrm{H}]^{+}\right)$: 226.0611. Found: 226.0608 .

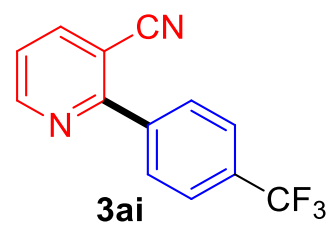

2-(4-(Trifluoromethyl)phenyl)nicotinonitrile (3ai). Purification by silica column chromatography $(10 \% \rightarrow 15 \%$ EtOAc in hexanes) afforded 3ai as a white solid (42.8 mg, $58 \%$ yield). $\mathrm{R}_{f}=0.32$ (33\% EtOAc in hexanes); ${ }^{1} \mathrm{H}$ NMR $\left(500 \mathrm{MHz}, \mathrm{CDCl}_{3}\right) \delta 8.94$ (dd, $J=4.8,1.6 \mathrm{~Hz}, 1 \mathrm{H}), 8.14(\mathrm{dd}, J=7.9,1.6 \mathrm{~Hz}, 1 \mathrm{H}), 8.08(\mathrm{~d}, J=8.0 \mathrm{~Hz}, 2 \mathrm{H}), 7.82(\mathrm{~d}, J=$ $8.0 \mathrm{~Hz}, 2 \mathrm{H}), 7.48(\mathrm{dd}, J=8.0,4.8 \mathrm{~Hz}, 1 \mathrm{H}){ }^{1} \mathrm{H}$ NMR $\left(400 \mathrm{MHz}, \mathrm{CDCl}_{3}\right) \delta 8.92-8.91(\mathrm{~m}$, $1 \mathrm{H}), 8.12(\mathrm{dd}, J=8.0,1.2 \mathrm{~Hz}, 1 \mathrm{H}), 8.06(\mathrm{~d}, J=8.0 \mathrm{~Hz}, 2 \mathrm{H}), 7.80$ (d, $J=8.0 \mathrm{~Hz}, 2 \mathrm{H})$, $7.46(\mathrm{dd}, J=8.0,4.8 \mathrm{~Hz}, 1 \mathrm{H}) ;{ }^{13} \mathrm{C} \mathrm{NMR}\left(125 \mathrm{MHz}, \mathrm{CDCl}_{3}\right) \delta 159.4,152.8,141.8,140.3$, 132.4, 131.9 (q, $J=42.5 \mathrm{~Hz}), 125.7,123.8$ (q, $J=270.8 \mathrm{~Hz}), 122.3,117.1$, 107.8. HRMS-ESI $(\mathrm{m} / \mathrm{z})$ calcd for $\mathrm{C}_{13} \mathrm{H}_{8} \mathrm{~F}_{3} \mathrm{~N}_{2}\left([\mathrm{M}+\mathrm{H}]^{+}\right): 249.0634$. Found: 249.0631 . 
<smiles>Cc1cccc(-c2ncccc2C#N)c1</smiles>

2-(m-Tolyl)nicotinonitrile (3aj). Purification by silica column chromatography $(5 \% \rightarrow 10 \%$ EtOAc in hexanes) afforded 3aj as a light yellow solid $\left(41.3 \mathrm{mg}, 71 \%\right.$ yield. $\mathrm{R}_{f}=0.29$ (17\% EtOAc in hexanes); ${ }^{1} \mathrm{H}$ NMR $\left(400 \mathrm{MHz}, \mathrm{CDCl}_{3}\right) \delta 8.86(\mathrm{dd}, J=4.8,1.6 \mathrm{~Hz}, 1 \mathrm{H})$, $8.06(\mathrm{dd}, J=8.0 \mathrm{~Hz}, 1.6 \mathrm{~Hz}, 1 \mathrm{H}), 7.71(\mathrm{t}, J=6.0 \mathrm{~Hz}, 2 \mathrm{H}), 7.42(\mathrm{t}, J=8.0 \mathrm{~Hz}, 1 \mathrm{H}), 7.37-$ $7.32(\mathrm{~m}, 2 \mathrm{H}), 2.45(\mathrm{~s}, 3 \mathrm{H})$; Spectroscopic data was identical to that reported previously. ${ }^{9}$<smiles>N#Cc1cccnc1-c1cccc(Br)c1</smiles>

2-(3-Bromophenyl)nicotinonitrile (3ak). Purification by silica column chromatography $\left(5 \% \rightarrow 10 \%\right.$ EtOAc in hexanes) afforded 3 ak as a white solid (40.3 $\mathrm{mg}, 52 \%$ yield). $\mathrm{R}_{f}=$ $0.32\left(20 \%\right.$ EtOAc in hexanes); ${ }^{1} \mathrm{H}$ NMR $\left(400 \mathrm{MHz}, \mathrm{CDCl}_{3}\right) \delta 8.88(\mathrm{~d}, J=4.8 \mathrm{~Hz}, 1 \mathrm{H})$, 8.10-8.07 (m, 2H), $7.88(\mathrm{~d}, J=8.8 \mathrm{~Hz}, 1 \mathrm{H}), 7.65$ (d, $J=8.0 \mathrm{~Hz}, 1 \mathrm{H}), 7.43-7.38(\mathrm{~m}, 2 \mathrm{H})$; ${ }^{13} \mathrm{C}$ NMR $\left(100 \mathrm{MHz}, \mathrm{CDCl}_{3}\right) \delta 159.3,152.7,141.8,138.9,133.2,131.9,130.1,127.3$, 122.8, 122.1, 117.2, 107.6. IR (film): 2225, 1576, 1550, 1431, 1404, 1264, 1227, 1180, 1116, 1076, 1063, 1035, 998, 876, 829, 809, 798, 764, 727; HRMS-ESI (m/z) calcd for $\mathrm{C}_{12} \mathrm{H}_{8} \mathrm{BrN}_{2}\left([\mathrm{M}+\mathrm{H}]^{+}\right): 258.9865$. Found: 258.9865 .

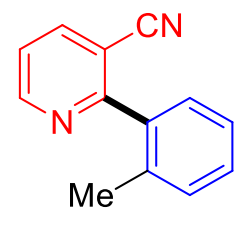

3al

2-(o-Tolyl)nicotinonitrile (3al). Purification by silica column chromatography (5\% EtOAc in hexanes) afforded 3al as a white solid (30.9 mg, 53\% yield). $\mathrm{R}_{f}=0.20$ (17\% EtOAc in hexanes); ${ }^{1} \mathrm{H}$ NMR $\left(400 \mathrm{MHz}, \mathrm{CDCl}_{3}\right) \delta 8.88-8.87(\mathrm{~m}, 1 \mathrm{H}), 8.08(\mathrm{dd}, J=8.0$, $1.6 \mathrm{~Hz}, 1 \mathrm{H}), 7.42-7.31(\mathrm{~m}, 5 \mathrm{H}), 2.26(\mathrm{~s}, 3 \mathrm{H})$; Spectroscopic data was identical to that reported previously. ${ }^{10}$ 


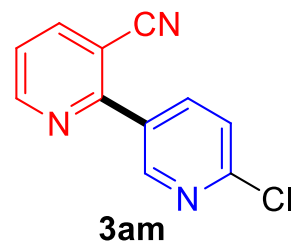

6'-Chloro-[2,3'-bipyridine]-3-carbonitrile (3am). Purification by silica column chromatography (20\% EtOAc in hexanes) afforded 3am as a white solid (27.4 mg, 42\% yield). $\mathrm{R}_{f}=0.21\left(30 \%\right.$ EtOAc in hexanes); ${ }^{1} \mathrm{H}$ NMR $\left(400 \mathrm{MHz}, \mathrm{CDCl}_{3}\right) \delta 8.98(\mathrm{~s}, 1 \mathrm{H})$, $8.92(\mathrm{~d}, J=4.8 \mathrm{~Hz}, 1 \mathrm{H}), 8.25$ (dd, $J=8.4,2.0 \mathrm{~Hz}, 1 \mathrm{H}), 8.12(\mathrm{~d}, J=8.0 \mathrm{~Hz}, 1 \mathrm{H}), 7.51-$ 7.45); ${ }^{13} \mathrm{C}$ NMR (100 MHz, $\left.\mathrm{CDCl}_{3}\right) \delta 156.9,153.1,153.0,149.9,141.9,138.7,131.8$, 124.1, 122.5, 116.9, 107.7. IR (film): $\mathrm{cm}^{-1} 2226,1590,1576,1566,1551,1405,1364$, 1103, 1039, 1010, 851, 807, 771, 752, 737; HRMS-ESI $(\mathrm{m} / \mathrm{z})$ calcd for $\mathrm{C}_{11} \mathrm{H}_{7} \mathrm{ClN}_{3}$ $\left([\mathrm{M}+\mathrm{H}]^{+}\right): 216.0323$. Found: 216.0324 .

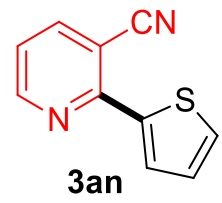

2-(Thiophen-2-yl)nicotinonitrile (3an). Purification by silica column chromatography $(5 \% \rightarrow 10 \%$ EtOAc in hexanes) afforded 3an as a light yellow solid ( $24.3 \mathrm{mg}, 44 \%$ yield). $\mathrm{R}_{f}=0.25$ (10\% EtOAc in hexanes); ${ }^{1} \mathrm{H}$ NMR $\left(400 \mathrm{MHz}, \mathrm{CDCl}_{3}\right) \delta 8.72(\mathrm{dd}, J=4.4,1.6$ $\mathrm{Hz}, 1 \mathrm{H}), 8.25$ (d, $J=4.4 \mathrm{~Hz}, 1 \mathrm{H}), 7.97(\mathrm{dd}, J=8.0,1.6 \mathrm{~Hz}, 1 \mathrm{H}), 7.53(\mathrm{~d}, J=5.2 \mathrm{~Hz}$, $1 \mathrm{H}), 7.22(\mathrm{dd}, J=8.0,5.2 \mathrm{~Hz}, 1 \mathrm{H}), 7.18-7.16(\mathrm{~m}, 1 \mathrm{H})$; Spectroscopic data was identical to that reported previously. ${ }^{11}$

III-c. Characterization of biaryl compounds 3ba-3ma (Scheme 3).

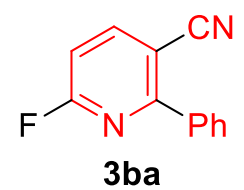

6-Fluoro-2-phenylnicotinonitrile (3ba). Purification by silica column chromatography (5\% EtOAc in hexanes) afforded 3ba as a white solid (29.0 mg, 49\% yield). $\mathrm{R}_{f}=0.42(17 \%$ EtOAc in hexanes); ${ }^{1} \mathrm{H}$ NMR (400 MHz, $\left.\mathrm{CDCl}_{3}\right) \delta 8.18-8.14(\mathrm{~m}, 1 \mathrm{H}), 7.99-7.97$ (m, 
2H), 7.55-7.53 (m, 3H), $7.02(\mathrm{dd}, J=8.4,3.9 \mathrm{~Hz}, 1 \mathrm{H}) ;{ }^{13} \mathrm{C} \mathrm{NMR}\left(100 \mathrm{MHz}, \mathrm{CDCl}_{3}\right) \delta$ 163.9 (d, $J=246.9 \mathrm{~Hz}), 161.3$ (d, $J=17.3 \mathrm{~Hz}), 146.9$ (d, $J=9.2 \mathrm{~Hz}), 135.5,131.0,128.8$ (d, $J=8.5 \mathrm{~Hz}), 116.9,108.6,108.2,104.6 ;{ }^{19} \mathrm{~F}$ NMR $\left(376 \mathrm{MHz}, \mathrm{CDCl}_{3}\right) \delta-56.88(\mathrm{~d}, J=$ $3.8 \mathrm{~Hz}$ ); IR (film): $\mathrm{cm}^{-1}$ 2226, 1587, 1562, 1435, 1401, 1382, 1298, 1258, 1228, 1192 , 1179, 1114, 1026, 908, 754, 723, 660, 641; HRMS-ESI (m/z) calcd for $\mathrm{C}_{12} \mathrm{H}_{8} \mathrm{FN}_{2}$ $\left([\mathrm{M}+\mathrm{H}]^{+}\right): 199.0666$. Found: 199.0664 .<smiles>N#Cc1ccc(C(F)(F)F)nc1-c1ccccc1</smiles>

2-Phenyl-6-(trifluoromethyl)nicotinonitrile (3ca). Purification by silica column chromatography (10\% EtOAc in hexanes) afforded 3ca as a light yellow oil (46.9 mg, $63 \%$ yield). $\mathrm{R}_{f}=0.5$ (12.5\% EtOAc in hexanes); ${ }^{1} \mathrm{H}$ NMR (400 MHz, $\left.\mathrm{CDCl}_{3}\right) \delta 8.27$ (d, $J$ $=8.0 \mathrm{~Hz}, 1 \mathrm{H}), 8.02-7.99(\mathrm{~m}, 2 \mathrm{H}), 7.74(\mathrm{~d}, J=8.0 \mathrm{~Hz}, 1 \mathrm{H}), 7.58-7.55(\mathrm{~m}, 4 \mathrm{H}) ;{ }^{19} \mathrm{~F} \mathrm{NMR}$ $\left(376 \mathrm{MHz}, \mathrm{CDCl}_{3}\right) \delta-68.66$; Spectroscopic data was identical to that reported previously. ${ }^{12}$

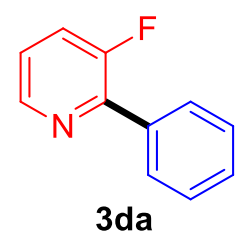

3-Fluoro-2-phenylpyridine (3da). Purification by silica column chromatography (5\% EtOAc in hexanes) afforded 3da as a colorless oil (31.8 mg, 61\% yield). $\mathrm{R}_{f}=0.31$ (10\% EtOAc in hexanes); ${ }^{1} \mathrm{H}$ NMR (400 MHz, $\left.\mathrm{CDCl}_{3}\right) \delta 8.51(\mathrm{~d}, J=4.4 \mathrm{~Hz}, 1 \mathrm{H}), 7.97-7.95$ (m, 2H), 7.50-7.42 (m, 4H), 7.26-7.22 (m, 1H); ${ }^{19} \mathrm{~F}$ NMR (376 MHz, $\left.\mathrm{CDCl}_{3}\right) \delta$-123.04; Spectroscopic data was identical to that reported previously. ${ }^{12}$

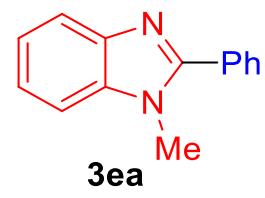


1-Methyl-2-phenyl-1H-benzo[d]imidazole (3ea). Purification by silica column chromatography $(50 \% \rightarrow 70 \%$ EtOAc in hexanes) afforded 3ea as a light yellow oil (44.3 mg, 71\% yield). $\mathrm{R}_{f}=0.22$ (EtOAc); ${ }^{1} \mathrm{H}$ NMR (400 MHz, $\left.\mathrm{CDCl}_{3}\right) \delta 7.83(\mathrm{dd}, J=6.0,3.2$ $\mathrm{Hz}, 1 \mathrm{H}), 7.80-7.76(\mathrm{~m}, 2 \mathrm{H}), 7.53-7.52(\mathrm{~m}, 3 \mathrm{H}), 7.42-7.38(\mathrm{~m}, 1 \mathrm{H}), 7.34-7.26(\mathrm{~m}, 2 \mathrm{H})$, $3.87(\mathrm{~s}, 3 \mathrm{H})$; Spectroscopic data was identical to that reported previously. ${ }^{13}$

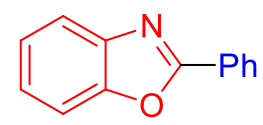

$3 \mathrm{fa}$

2-Phenylbenzo[d]oxazole (3fa). Purification by silica column chromatography (5\% EtOAc in hexanes) afforded $\mathbf{3 f a}$ as a white solid (29.9 mg, 51\% yield). $\mathrm{R}_{f}=0.65(20 \%$ EtOAc in hexanes); ${ }^{1} \mathrm{H}$ NMR $\left(500 \mathrm{MHz}, \mathrm{CDCl}_{3}\right) \delta 8.28-8.26(\mathrm{~m}, 2 \mathrm{H}), 7.78(\mathrm{dd}, J=6.0$, $3.0 \mathrm{~Hz}, 1 \mathrm{H}), 7.59(\mathrm{dd}, J=6.0,3.0 \mathrm{~Hz}, 1 \mathrm{H}), 7.54-7.53(\mathrm{~m}, 3 \mathrm{H}), 7.36(\mathrm{dd}, J=6.0,3.0 \mathrm{~Hz}$, $2 \mathrm{H})$; Spectroscopic data was identical to that reported previously. ${ }^{14}$

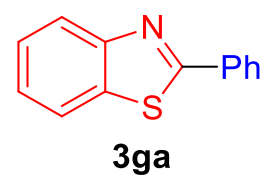

2-Phenylbenzo[d]thiazole (3ga). Purification by silica column chromatography (5\% EtOAc in hexanes) afforded 3 ga as a white solid (50.6 mg, 80\% yield). $\mathrm{R}_{f}=0.43(10 \%$ EtOAc in hexanes); ${ }^{1} \mathrm{H}$ NMR $\left(400 \mathrm{MHz}, \mathrm{CDCl}_{3}\right) \delta 8.12-8.07(\mathrm{~m}, 3 \mathrm{H}), 7.91(\mathrm{~d}, J=8.0 \mathrm{~Hz}$, $1 \mathrm{H}), 7.51-7.48(\mathrm{~m}, 4 \mathrm{H}), 7.39(\mathrm{t}, J=7.6 \mathrm{~Hz}, 1 \mathrm{H})$; Spectroscopic data was identical to that reported previously. ${ }^{15}$<smiles>Cn1c(=O)c2c(nc(-c3ccccc3)n2C)n(C)c1=O</smiles>

3ha

1,3,7-Trimethyl-8-phenyl-1H-purine-2,6(3H,7H)-dione (3ha). Purification by silica column chromatography $(50 \% \rightarrow 75 \%$ EtOAc in hexanes) afforded 3 ha as a white solid (63.8 mg, 78\% yield). $\mathrm{R}_{f}=0.14$ (50\% EtOAc in hexanes); ${ }^{1} \mathrm{H}$ NMR (400 MHz, acetone) 
$\delta 7.79(\mathrm{dd}, J=6.4,3.2 \mathrm{~Hz}, 2 \mathrm{H}), 7.55-7.52(\mathrm{~m}, 3 \mathrm{H}), 4.04(\mathrm{~s}, 3 \mathrm{H}), 3.48(\mathrm{~s}, 3 \mathrm{H}), 3.27$ (s, $3 \mathrm{H})$; Spectroscopic data was identical to that reported previously. ${ }^{16}$

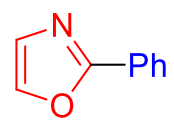

3ia

2-Phenyloxazole (3ia). Purification by silica column chromatography (5\% EtOAc in hexanes) afforded 3ia as a light yellow solid (25.3 mg, 58\% yield). $\mathrm{R}_{f}=0.27$ (12.5\% EtOAc in hexanes); ${ }^{1} \mathrm{H}$ NMR (400 MHz, $\left.\mathrm{CDCl}_{3}\right) \delta 8.06-8.04(\mathrm{~m}, 2 \mathrm{H}), 7.71(\mathrm{~d}, J=0.8$ $\mathrm{Hz}, 1 \mathrm{H}), 7.49-7.44(\mathrm{~m}, 3 \mathrm{H}), 7.24(\mathrm{~s}, 1 \mathrm{H})$; Spectroscopic data was identical to that reported previously. ${ }^{17}$

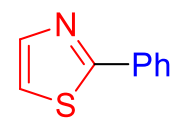

3ja

2-Phenylthiazole (3ja). Purification by silica column chromatography (10\% EtOAc in hexanes) afforded 3ja as a light yellow oil (27.4 mg, 57\% yield). $\mathrm{R}_{f}=0.32(17 \%$ EtOAc in hexanes); ${ }^{1} \mathrm{H}$ NMR (500 MHz, $\left.\mathrm{CDCl}_{3}\right) \delta 7.98-7.96(\mathrm{~m}, 2 \mathrm{H}), 7.87(\mathrm{~d}, J=3.5 \mathrm{~Hz}, 1 \mathrm{H})$, $7.45-7.43(\mathrm{~m}, 3 \mathrm{H}), 7.33(\mathrm{~d}, J=3.5 \mathrm{~Hz}, 1 \mathrm{H})$; Spectroscopic data was identical to that reported previously. ${ }^{18}$<smiles>Clc1cnc(-c2ccccc2)c(Cl)n1</smiles>

3,5-Dichloro-2-phenylpyrazine (3ka). Purification by silica column chromatography (1\% EtOAc in hexanes) afforded $\mathbf{3 k a}$ as a white solid (50.4 mg, 75\% yield). $\mathrm{R}_{f}=0.4$ (hexanes); ${ }^{1} \mathrm{H}$ NMR (400 MHz, $\left.\mathrm{CDCl}_{3}\right) \delta 8.59(\mathrm{~s}, 1 \mathrm{H}), 7.80-7.78(\mathrm{~m}, 2 \mathrm{H}), 7.51-7.48(\mathrm{~m}, 3 \mathrm{H})$; Spectroscopic data was identical to that reported previously. ${ }^{19}$ 


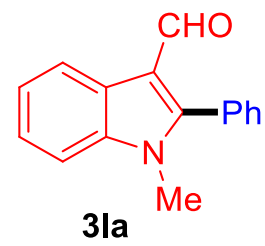

1-Methyl-2-phenyl-1H-indole-3-carbaldehyde (3la). Purification by silica column chromatography $(10 \% \rightarrow 30 \%$ EtOAc in hexanes) afforded 3la as a red solid $(20.9 \mathrm{mg}, 30 \%$ yield). $\mathrm{R}_{f}=0.54$ (44\% EtOAc in hexanes); ${ }^{1} \mathrm{H}$ NMR $\left(400 \mathrm{MHz}, \mathrm{CDCl}_{3}\right) \delta 9.72(\mathrm{~s}, 1 \mathrm{H})$, $8.42(\mathrm{dd}, J=6.0,2.8 \mathrm{~Hz}, 1 \mathrm{H}), 7.55$ (dd, $J=6.4,2.8 \mathrm{~Hz}, 3 \mathrm{H}), 7.48$ (dd, $J=6.4,2.8 \mathrm{~Hz}$, 2H), 7.41-7.34 (m, 4H), $3.66(\mathrm{~s}, 3 \mathrm{H})$.; Spectroscopic data was identical to that reported previously. ${ }^{20}$<smiles>N#Cc1cc(F)cc(F)c1-c1ccccc1</smiles>

4,6-Difluoro-[1,1'-biphenyl]-2-carbonitrile (3ma). Purification by silica column chromatography $(5 \% \rightarrow 10 \%$ EtOAc in hexanes) afforded $\mathbf{3 m a}$ as a white solid $(27.9 \mathrm{mg}$, $43 \%$ yield). $\mathrm{R}_{f}=0.24$ (12.5\% EtOAc in hexanes); ${ }^{1} \mathrm{H} \mathrm{NMR}\left(500 \mathrm{MHz}, \mathrm{CDCl}_{3}\right) \delta 7.59-$ $7.51(\mathrm{~m}, 6 \mathrm{H}), 7.32-7.27(\mathrm{~m}, 1 \mathrm{H}) ;{ }^{19} \mathrm{~F}$ NMR $\left(376 \mathrm{MHz}, \mathrm{CDCl}_{3}\right) \delta-126.8$ to $-126.9(\mathrm{~m}),-$ 137.3 to $-137.4(\mathrm{~m}){ }^{13} \mathrm{C}$ NMR $\left(100 \mathrm{MHz}, \mathrm{CDCl}_{3}\right) \delta 153.6(\mathrm{dd}, J=257.6,13.7 \mathrm{~Hz}), 148.0$ (dd, $J=250.2,13.3 \mathrm{~Hz}), 135.8$ (dd, $J=14.7,1.6 \mathrm{~Hz}), 130.34$ (d, $J=2.5 \mathrm{~Hz}), 130.00$ (dd, $J=8.0,4.8 \mathrm{~Hz}), 129.72,129.57,129.55,128.74,117.07$ (dd, $J=18.7,0.9 \mathrm{~Hz}), 109.34$ (dd, $J=3.7,3.0 \mathrm{~Hz}$ ); IR (film): $\mathrm{cm}^{-1}$ 2240, 1617, 1487, 1450, 1438, 1291, 1271, 1180, 1156, 1107, 1076, 1027, 976, 956, 881, 826, 697, 674; HRMS-ESI (m/z) calcd for $\mathrm{C}_{13} \mathrm{H}_{6} \mathrm{~F}_{2} \mathrm{~N}\left([\mathrm{M}-\mathrm{H}]^{+}\right): 214.0474$. Found: 214.0477.

\section{III-d. Characterization of vinylated and alkynylated products (Scheme 4).}

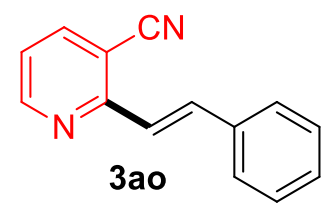


(E)-2-Styrylnicotinonitrile (3ao). Purification by silica column chromatography $(5 \% \rightarrow 10 \%$ EtOAc in hexanes) afforded $3 \mathbf{a o}$ as a light yellow solid (44.9 $\mathrm{mg}, 73 \%$ yield). $\mathrm{R}_{f}=0.43$ (30\% EtOAc in hexanes); ${ }^{1} \mathrm{H}$ NMR $\left(400 \mathrm{MHz}, \mathrm{CDCl}_{3}\right) \delta 8.72(\mathrm{~d}, J=4.8 \mathrm{~Hz}$, 1H), 7.99 (d, $J=15.6 \mathrm{~Hz}, 1 \mathrm{H}), 7.87$ (d, $J=7.2 \mathrm{~Hz}, 1 \mathrm{H}), 7.61$ (d, $J=7.2 \mathrm{~Hz}, 2 \mathrm{H}), 7.47$ (d, $J=15.6 \mathrm{~Hz}, 1 \mathrm{H}), 7.38-7.32(\mathrm{~m}, 3 \mathrm{H}), 7.19$ (dd, $J=8.0,4.8 \mathrm{~Hz}, 1 \mathrm{H}) ;{ }^{13} \mathrm{C}$ NMR $(100$ $\left.\mathrm{MHz}, \mathrm{CDCl}_{3}\right) \delta 157.3,152.6,140.4,137.8,135.5,129.5,128.8,127.8,122.6,121.3$, 116.6, 107.4. IR (film): 2221, 1632, 1573, 1555, 1447, 1424, 1199, 1178, 1090, 1071, 1028, 970, 857, 734, 684; HRMS-ESI (m/z) calcd for $\mathrm{C}_{14} \mathrm{H}_{11} \mathrm{~N}_{2}\left([\mathrm{M}+\mathrm{H}]^{+}\right): 207.0917$. Found: 207.0914 .

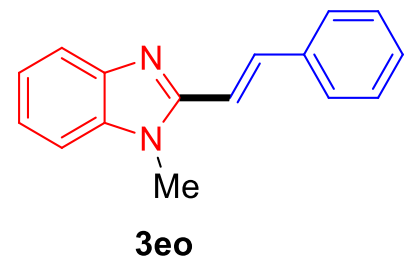

(E)-1-Methyl-2-styryl-1H-benzo[d]imidazole (3eo). Purification by silica column chromatography (50\% EtOAc in hexanes) afforded 3 eo as a yellow solid (43.9 mg, 62\% yield). $\mathrm{R}_{f}=0.19(100 \% \mathrm{EtOAc}) ;{ }^{1} \mathrm{H}$ NMR $\left(400 \mathrm{MHz}, \mathrm{CDCl}_{3}\right) \delta 7.99(\mathrm{~d}, J=16.0 \mathrm{~Hz}$, $1 \mathrm{H}), 7.79-7.75(\mathrm{~m}, 1 \mathrm{H}), 7.61(\mathrm{~d}, J=7.2 \mathrm{~Hz}, 2 \mathrm{H}), 7.41-7.38(\mathrm{~m}, 2 \mathrm{H}), 7.35-7.32(\mathrm{~m}, 2 \mathrm{H})$, 7.28-7.26 (m, 2H), $7.10(\mathrm{~d}, J=16.0 \mathrm{~Hz}, 1 \mathrm{H}), 3.87(\mathrm{~s}, 3 \mathrm{H})$. Spectroscopic data was identical to that reported previously. ${ }^{21}$

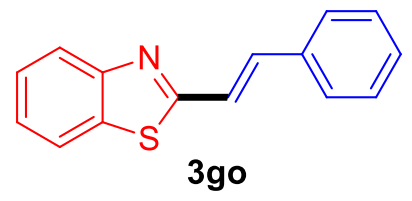

(E)-2-Styrylbenzo[d]thiazole (3go). Purification by silica column chromatography ( $5 \% \rightarrow 10 \%$ EtOAc in hexanes) afforded $3 \mathrm{go}$ as a yellow solid $\left(61.3 \mathrm{mg}, 86 \%\right.$ yield). $\mathrm{R}_{f}=$ 0.27 (9\% EtOAc in hexanes); ${ }^{1} \mathrm{H}$ NMR (400 MHz, $\left.\mathrm{CDCl}_{3}\right) \delta 7.99(\mathrm{~d}, J=8.0 \mathrm{~Hz}, 1 \mathrm{H})$, $7.84(\mathrm{~d}, J=8.0 \mathrm{~Hz}, 1 \mathrm{H}), 7.57$ (d, $J=8.0 \mathrm{~Hz}, 2 \mathrm{H}), 7.51$ (d, $J=16.4 \mathrm{~Hz}, 1 \mathrm{H}), 7.46$ (t, $J=$ $8.0 \mathrm{~Hz}, 1 \mathrm{H}), 7.42-7.34(\mathrm{~m}, 5 \mathrm{H})$. Spectroscopic data was identical to that reported previously. ${ }^{21}$ 


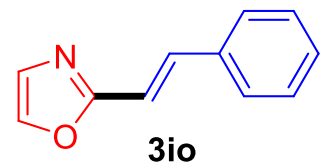

(E)-2-Styryloxazole (3io). Purification by silica column chromatography $(10 \% \rightarrow 20 \%$ EtOAc in hexanes) afforded $3 i o$ as a light yellow oil (40.4 mg, 79\% yield). $\mathrm{R}_{f}=0.39$ (23\% EtOAc in hexanes); ${ }^{1} \mathrm{H}$ NMR (400 $\left.\mathrm{MHz} \mathrm{CDCl}_{3}\right) \delta 7.61(\mathrm{~s}, 1 \mathrm{H}), 7.52-7.48(\mathrm{~m}, 4 \mathrm{H})$, 7.39-7.32 (m, 4H), $7.17(\mathrm{~s}, 1 \mathrm{H}), 6.95(\mathrm{~d}, J=16.4 \mathrm{~Hz}, 1 \mathrm{H})$. Spectroscopic data was identical to that reported previously. ${ }^{21}$

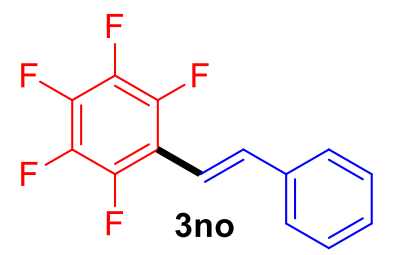

(E)-1,2,3,4,5-Pentafluoro-6-styrylbenzene (3no). Purification by silica column chromatography (pentane) afforded 3no as a white solid $\left(60.1 \mathrm{mg}, 74 \%\right.$ yield). $\mathrm{R}_{f}=0.50$ (in hexanes); ${ }^{1} \mathrm{H}$ NMR (400 MHz, $\left.\mathrm{CDCl}_{3}\right) \delta 7.52-7.49(\mathrm{~m}, 2 \mathrm{H}), 7.44-7.27(\mathrm{~m}, 4 \mathrm{H}), 6.97$ $(\mathrm{d}, J=16.8 \mathrm{~Hz}, 1 \mathrm{H}) ;{ }^{19} \mathrm{~F}$ NMR $\left(376 \mathrm{MHz}, \mathrm{CDCl}_{3}\right) \delta-142.82(\mathrm{dd}, J=21.5,7.7 \mathrm{~Hz})$, 156.53 to $-156.71(\mathrm{~m}),-163.03(\mathrm{dt}, J=21.4,7.7 \mathrm{~Hz})$. Spectroscopic data was identical to that reported previously. ${ }^{22}$<smiles>N#Cc1ccc(F)c(F)c1/C=C/c1ccccc1</smiles>

(E)-3,4-Difluoro-2-styrylbenzonitrile (30o). Purification by silica column chromatography $(5 \% \rightarrow 10 \%$ EtOAc in hexanes) afforded 300 as a white solid $(60.7 \mathrm{mg}$, $84 \%$ yield). $\mathrm{R}_{f}=0.44$ (11\% EtOAc in hexanes); ${ }^{1} \mathrm{H}$ NMR (400 MHz, $\left.\mathrm{CDCl}_{3}\right) \delta 7.62-7.52$ (m, 3H), 7.46-7.33 (m, 4H), $7.21(\mathrm{~d}, J=16.4 \mathrm{~Hz}, 1 \mathrm{H}), 7.11(\mathrm{dd}, J=16.4,7.6 \mathrm{~Hz}, 1 \mathrm{H})$; ${ }^{13} \mathrm{C} \mathrm{NMR}\left(100 \mathrm{MHz}, \mathrm{CDCl}_{3}\right) \delta 165.1,159.4,158.8,142.6,140.6,135.6,133.31,133.28$, $129.56,129.47,129.41,127.6,127.3,125.1,124.5,119.9,118.5,117.80,117.8,116.2$, 116.0, 105.3; ${ }^{19} \mathrm{~F}$ NMR (376 MHz, $\left.\mathrm{CDCl}_{3}\right) \delta-127.17$ to $-127.27(\mathrm{~m}),-130.05--130.13$ 
(m). IR (film): 1610, 1489, 1438, 1284, 1248, 1219, 1031, 823, 760, 726, 689; HRMS-EI $(\mathrm{m} / \mathrm{z})$ calcd for $\mathrm{C}_{15} \mathrm{H}_{9} \mathrm{~F}_{2} \mathrm{~N}\left([\mathrm{M}]^{+}\right)$: 241.0703. Found: 241.0702 .

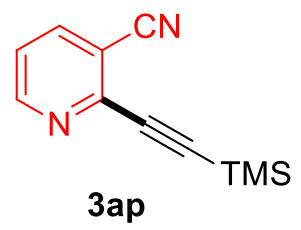

2-((Trimethylsilyl)ethynyl)nicotinonitrile (3ap). Purification by silica column chromatography (10\% EtOAc in hexanes) afforded 3ap as a light yellow solid (49.8 mg, $83 \%$ yield). $\mathrm{R}_{f}=0.29$ (17\% EtOAc in hexanes); ${ }^{1} \mathrm{H}$ NMR (400 MHz, $\left.\mathrm{CDCl}_{3}\right) \delta 8.84$ (d, $J$ $=4.8 \mathrm{~Hz}, 1 \mathrm{H}), 7.99(\mathrm{~d}, J=8.0 \mathrm{~Hz}, 1 \mathrm{H}), 7.40(\mathrm{dd}, J=8.0,4.8 \mathrm{~Hz}, 1 \mathrm{H}), 0.28(\mathrm{~s}, 9 \mathrm{H})$; Spectroscopic data was identical to that reported previously. ${ }^{23}$<smiles>Cn1c(C#C[As])nc2ccccc21</smiles>

1-Methyl-2-((trimethylsilyl)ethynyl)-1H-benzo[d]imidazole (3ep). Purification by silica column chromatography $(10 \% \rightarrow 20 \%$ EtOAc in hexanes) afforded 3 ep as a yellow solid (33.9 mg, 50\% yield). $\mathrm{R}_{f}=0.26$ (25\% EtOAc in hexanes); ${ }^{1} \mathrm{H}$ NMR $(400 \mathrm{MHz}$, $\left.\mathrm{CDCl}_{3}\right) \delta 7.73(\mathrm{dt}, J=7.2,1.2 \mathrm{~Hz}, 1 \mathrm{H}), 7.32-7.26(\mathrm{~m}, 3 \mathrm{H}), 3.84(\mathrm{~s}, 3 \mathrm{H}), 0.31(\mathrm{~s}, 9 \mathrm{H}) ;{ }^{13} \mathrm{C}$ NMR (100 MHz, $\left.\mathrm{CDCl}_{3}\right) \delta 142.6,137.2,134.6,123.9,122.8,120.2,109.4,102.1,93.3$, 30.6, -0.5. IR (film): 1456, 1435, 1381, 1330, 1285, 1249, 879, 839, 763, 742, 637, 628; HRMS-ESI (m/z) calcd for $\mathrm{C}_{13} \mathrm{H}_{17} \mathrm{~N}_{2} \mathrm{Si}\left([\mathrm{M}+\mathrm{H}]^{+}\right)$: 229.1156. Found: 229.1155.

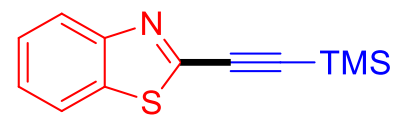

$3 g p$

2-((Trimethylsilyl)ethynyl)benzo[d]thiazole (3gp). Purification by silica column chromatography ( $5 \rightarrow 10 \%$ EtOAc in hexanes) afforded $3 \mathrm{gp}$ as a light yellow oil $(55.7 \mathrm{mg}$, $80 \%$ yield). $\mathrm{R}_{f}=0.47$ (9\% EtOAc in hexanes); ${ }^{1} \mathrm{H}$ NMR (400 MHz, $\left.\mathrm{CDCl}_{3}\right) \delta 8.03$ (d, $J=$ $8.0 \mathrm{~Hz}, 1 \mathrm{H}), 7.82(\mathrm{~d}, J=8.0 \mathrm{~Hz}, 1 \mathrm{H}), 7.48(\mathrm{t}, J=8.0 \mathrm{~Hz}, 1 \mathrm{H}), 7.42(\mathrm{t}, J=8.0 \mathrm{~Hz}, 1 \mathrm{H})$, 
$0.29(\mathrm{~s}, 9 \mathrm{H})$. Spectroscopic data was identical to that reported previously. ${ }^{24}$

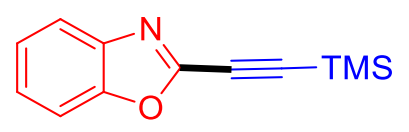

$3 \mathrm{fp}$

2-((Trimethylsilyl)ethynyl)benzo[d]oxazole (3fp). Purification by silica column chromatography $(5 \% \rightarrow 10 \%$ EtOAc in hexanes) afforded $\mathbf{3 f p}$ as a light yellow oil (49.2 mg, 76\% yield). $\mathrm{R}_{f}=0.57$ (9\% EtOAc in hexanes); ${ }^{1} \mathrm{H} \mathrm{NMR}\left(400 \mathrm{MHz}, \mathrm{CDCl}_{3}\right) \delta 7.71-$ $7.68(\mathrm{~m}, 1 \mathrm{H}), 7.49-7.46(\mathrm{~m}, 1 \mathrm{H}), 7.37-7.33(\mathrm{~m}, 2 \mathrm{H}), 0.29(\mathrm{~s}, 9 \mathrm{H}) ;{ }^{13} \mathrm{C}$ NMR $(100 \mathrm{MHz}$, $\left.\mathrm{CDCl}_{3}\right) \delta 150.0,146.9,140.6,126.4,125.0,120.5,110.6,101.6,91.4,-0.79$. IR (film): 1532, 1449, 1251, 1236, 1140, 1107, 945, 860, 832, 760, 742, 722, 624; HRMS-ESI (m/z) calcd for $\mathrm{C}_{12} \mathrm{H}_{14} \mathrm{OSiN}\left([\mathrm{M}+\mathrm{H}]^{+}\right)$: 216.0839. Found: 216.0842 .
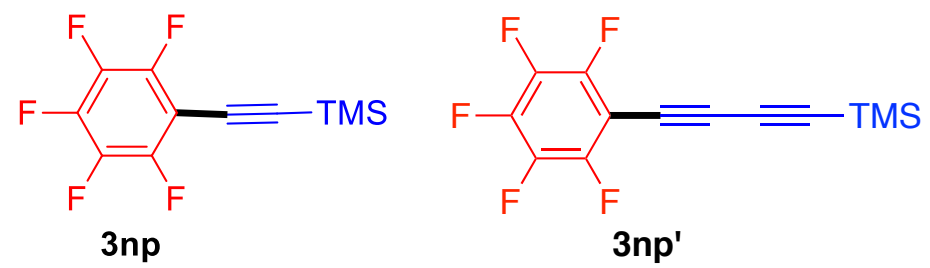

Trimethyl((perfluorophenyl)ethynyl)silane (3np) and Trimethyl((perfluorophenyl) buta-1,3-diyn-1-yl)silane (3np'). Purification by silica column chromatography (pentane) afforded 3np as a mixture containing Hiyama type coupling product 3np' (9:1) as a white solid (58.3 mg, 74\% yield). $\mathrm{R}_{f}=0.72$ (in pentane); ${ }^{1} \mathrm{H}$ NMR (400 MHz, $\left.\mathrm{CDCl}_{3}\right) \delta 0.19(\mathrm{~s}, 9 \mathrm{H}) ;{ }^{19} \mathrm{~F}$ NMR $\left(376 \mathrm{MHz}, \mathrm{CDCl}_{3}\right) \delta-135.85$ to $-135.93(\mathrm{~m}),-152.51(\mathrm{t}$, $J=20.8 \mathrm{~Hz}$ ), -161.95 to -162.09 (qd, $J=9.1,2.2 \mathrm{~Hz}$ ). Spectroscopic data was identical to that reported previously. ${ }^{25}$

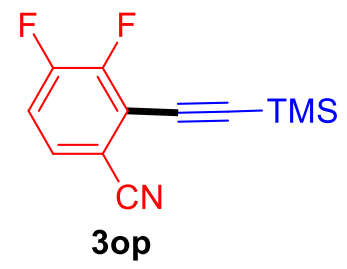

3,4-Difluoro-2-((trimethylsilyl)ethynyl)benzonitrile (3op). Purification by silica column chromatography $(5 \% \rightarrow 10 \%$ EtOAc in hexanes) afforded 3op as a white solid 
(43.1 mg, 61\% yield). $\mathrm{R}_{f}=0.35$ (9\% EtOAc in hexanes); ${ }^{1} \mathrm{H}$ NMR $\left(400 \mathrm{MHz}, \mathrm{CDCl}_{3}\right) \delta$ 7.42-7.38 (m, 1H), 7.24-7.17 (m, 1H), 0.29 (s, 9H); ${ }^{13} \mathrm{C}$ NMR $\left(100 \mathrm{MHz}, \mathrm{CDCl}_{3}\right) \delta$ $153.1(\mathrm{dd}, J=257.6, J=12.4 \mathrm{~Hz}), 152.1(\mathrm{dd}, J=256.1, J=14.0 \mathrm{~Hz}), 129.19-129.05$ (m), 118.47-118.29 (m), 117.92 (d, $J=18.6 \mathrm{~Hz}), 115.7-115.6$ (m), 112.7-112.6(m), $110.3(\mathrm{~d}, J=4.3 \mathrm{~Hz}), 92.5$ (d, $J=3.8 \mathrm{~Hz}),-0.6 ;{ }^{19} \mathrm{~F} \mathrm{NMR}\left(376 \mathrm{MHz}, \mathrm{CDCl}_{3}\right) \delta-127.22$ (ddd, $J=21.2,7.3,4.5 \mathrm{~Hz}$ ), -130.09 (dd, $J=21.2,7.3 \mathrm{~Hz}$ ). IR (film): 1610, 1489, 1438, $1284,1248,1219,1031,823,760,726,687$; HRMS-ESI $(\mathrm{m} / \mathrm{z})$ calcd for $\mathrm{C}_{12} \mathrm{H}_{10} \mathrm{~F}_{2} \mathrm{NSi}$ $\left([\mathrm{M}]^{+}\right): 235.0629$. Found: 235.0624 .

\section{Synthesis and characterization of 3ca, 3cd, 3ce and 3aq (Scheme 5).}

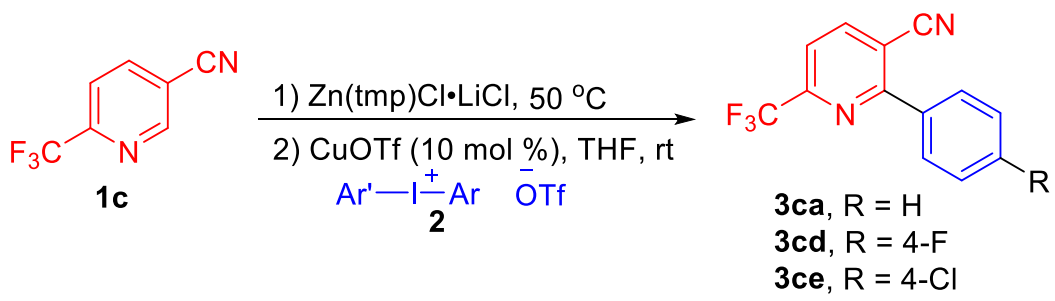

To an oven dried Schlenk tube was added compound 1c (51.6 mg, $0.3 \mathrm{mmol}, 1.0$ equiv) and the solution of $\mathrm{Zn}(\mathrm{tmp}) \mathrm{Cl} \cdot \mathrm{LiCl}\left(0.6 \mathrm{mmol}, 2.0\right.$ equiv) in THF $(3 \mathrm{~mL})$ under $\mathrm{N}_{2}$. The mixture was allowed to stir at $50{ }^{\circ} \mathrm{C}$ for $1 \mathrm{~h}$. To the reaction mixture, was added iodonium salt 2 (0.6 mmol, 2.0 equiv), CuOTf•1/2 PhMe (7.8 mg, $0.03 \mathrm{mmol}, 10 \mathrm{~mol} \%)$ and anhydrous THF $(3.0 \mathrm{~mL})$ under $\mathrm{N}_{2}$. The resulting mixture was allowed to stir at room temperature. Upon the complete consumption of $\mathbf{1}$ (monitored by TLC), the reaction was quenched by the addition of $\mathrm{MeOH}(1 \mathrm{~mL})$. Then the mixture was filtered though a plug of $\mathrm{Al}_{2} \mathrm{O}_{3}$ and washed with $\mathrm{Et}_{2} \mathrm{O}(5 \mathrm{~mL} \times 3)$ and $\mathrm{CH}_{2} \mathrm{Cl}_{2}(5 \mathrm{~mL} \times 3)$. The combined organic layers were concentrated in vacuo. Purification of the crude mixture by silica column chromatography afforded product 3.<smiles>N#Cc1ccc(C(F)(F)F)nc1-c1ccccc1</smiles>

Characterization of $\mathbf{3 c a}$ is presented on the page $\mathrm{S} 9$ above. 


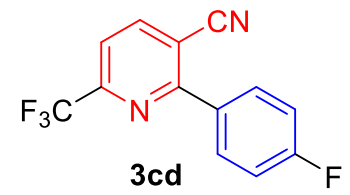

2-(4-Fluorophenyl)-6-(trifluoromethyl)nicotinonitrile (3cd). Purification by silica column chromatography (5\% EtOAc in hexanes) afforded 3cd as a white solid (33.5 mg, $42 \%$ yield). $\mathrm{R}_{f}=0.52\left(17 \%\right.$ EtOAc in hexanes); ${ }^{1} \mathrm{H}$ NMR $\left(500 \mathrm{MHz}, \mathrm{CDCl}_{3}\right) \delta 8.29(\mathrm{~d}, J$ $=8.0 \mathrm{~Hz}, 1 \mathrm{H}), 8.08-8.05(\mathrm{~m}, 2 \mathrm{H}), 7.77(\mathrm{dd}, J=8.0,2.5 \mathrm{~Hz}, 1 \mathrm{H}), 7.28-7.25(\mathrm{~m}, 2 \mathrm{H}) ;{ }^{13} \mathrm{C}$ NMR $\left(125 \mathrm{MHz}, \mathrm{CDCl}_{3}\right) \delta 164.5(\mathrm{~d}, J=200.8 \mathrm{~Hz}), 160.0,150.6$ (q, $\left.J=28.6 \mathrm{~Hz}\right), 143.9$, $131.8,131.3(\mathrm{~d}, J=17.0 \mathrm{~Hz}), 120.5(\mathrm{q}, J=219.8 \mathrm{~Hz}), 118.1,116.5,116.0(\mathrm{~d}, J=15.5$ $\mathrm{Hz}), 109.7 ;{ }^{19} \mathrm{~F}$ NMR (376 MHz, $\left.\mathrm{CDCl}_{3}\right) \delta-68.72,-108.60$ to -108.66 (m). IR (film): $\mathrm{cm}^{-1} 3328,1602,1512,1396,1337,1227,1202,1141,1128,1108,1085,1042,1015$, 864, 842, 809, 787, 744, 727, 694; HRMS (ESI) calcd for $\mathrm{C}_{13} \mathrm{H}_{7} \mathrm{~F}_{4} \mathrm{~N}_{2}\left([\mathrm{M}+\mathrm{H}]^{+}\right)$: 267.0540. Found: 267.0540.

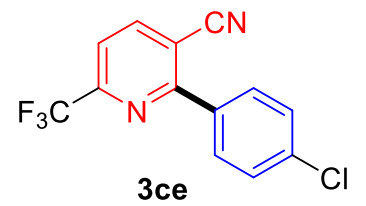

2-(4-Chlorophenyl)-6-(trifluoromethyl)nicotinonitrile (3ce). Purification by silica column chromatography (5\% EtOAc in hexanes) afforded 3ce as a white solid $(34.5 \mathrm{mg}$, $41 \%$ yield). $\mathrm{R}_{f}=0.35$ (11\% EtOAc in hexanes); ${ }^{1} \mathrm{H}$ NMR $\left(400 \mathrm{MHz}, \mathrm{CDCl}_{3}\right) \delta 8.28(\mathrm{~d}, J$ $=8.0 \mathrm{~Hz}, 1 \mathrm{H}), 7.98(\mathrm{~d}, J=8.4 \mathrm{~Hz}, 2 \mathrm{H}), 7.76(\mathrm{~d}, J=8.0 \mathrm{~Hz}, 1 \mathrm{H}), 7.54(\mathrm{~d}, J=8.4 \mathrm{~Hz}, 2 \mathrm{H})$; ${ }^{13} \mathrm{C} \mathrm{NMR}\left(125 \mathrm{MHz}, \mathrm{CDCl}_{3}\right) \delta 159.9,150.7$ (q, $\left.J=28.8 \mathrm{~Hz}\right), 143.9,137.6,134.0,130.4$, 129.2, 120.5 (q, $J=218.6 \mathrm{~Hz}$ ), 118.3, 116.4, 109.8; IR (film): $\mathrm{cm}^{-1} 1595,1572,1409$, $1395,1339,1237,1204,1174,1114,1086,1045,1012,860,841,784,745,724,683$; HRMS-ESI (m/z) calcd for $\mathrm{C}_{13} \mathrm{H}_{7} \mathrm{~F}_{3} \mathrm{ClN}_{2}\left([\mathrm{M}+\mathrm{H}]^{+}\right)$: 283.0244. Found: 283.0248.
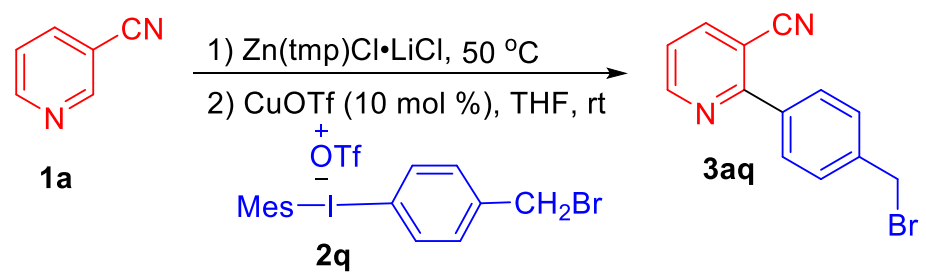
To an oven dried Schlenk tube was added compound 1a (31.2 mg, $0.3 \mathrm{mmol}, 1.0$ equiv) and the solution of $\mathrm{Zn}(\mathrm{tmp}) \mathrm{Cl} \cdot \mathrm{LiCl}\left(0.6 \mathrm{mmol}, 2.0\right.$ equiv) in THF $(3 \mathrm{~mL})$ under $\mathrm{N}_{2}$. The mixture was allowed to stir at $50{ }^{\circ} \mathrm{C}$ for $1 \mathrm{~h}$. To the reaction mixture, was added iodonium salt 2q (339.0 mg, $0.6 \mathrm{mmol}, 2.0$ equiv), CuOTf•1/2 PhMe (7.8 mg, $0.03 \mathrm{mmol}, 10 \mathrm{~mol}$ $\%)$ and anhydrous THF $(3.0 \mathrm{~mL})$ under $\mathrm{N}_{2}$. The resulting mixture was allowed to stir at room temperature. Upon the complete consumption of 1a (monitored by TLC), the reaction was quenched by the addition of $\mathrm{MeOH}(1 \mathrm{~mL})$. Then the mixture was filtered though a plug of $\mathrm{Al}_{2} \mathrm{O}_{3}$ and washed with $\mathrm{Et}_{2} \mathrm{O}(5 \mathrm{~mL} \times 3)$ and $\mathrm{CH}_{2} \mathrm{Cl}_{2}(5 \mathrm{~mL} \times 3)$. The combined organic layers were concentrated in vacuo. Purification of the crude mixture by silica column chromatography $(30 \% \rightarrow 50 \%$ EtOAc in hexanes) afforded $\mathbf{3 a q}$ as a white solid (47.5 mg, 58\% yield). $\mathrm{R}_{f}=0.33$ (50\% EtOAc in hexanes); ${ }^{1} \mathrm{H}$ NMR (400 MHz, $\left.\mathrm{CDCl}_{3}\right) \delta 8.87(\mathrm{~d}, J=4.8 \mathrm{~Hz}, 1 \mathrm{H}), 8.07(\mathrm{~d}, J=8.0 \mathrm{~Hz}, 1 \mathrm{H}), 7.92(\mathrm{~d}, J=8.0 \mathrm{~Hz}, 2 \mathrm{H}), 7.55$ $(\mathrm{d}, J=8.0 \mathrm{~Hz}, 2 \mathrm{H}), 7.38(\mathrm{dd}, J=8.0,4.8 \mathrm{~Hz}, 1 \mathrm{H}), 4.55(\mathrm{~s}, 2 \mathrm{H}) ;{ }^{13} \mathrm{C}$ NMR $(100 \mathrm{MHz}$, $\left.\mathrm{CDCl}_{3}\right) \delta 160.1,152.6,141.8,139.8,137.0,129.31,129.26,121.7,117.5,107.4,32.5$; IR (film): $\mathrm{cm}^{-1}$ 2226, 1552, 1432, 1407, 1224, 1202, 1180, 1099, 1017, 883, 809, 772, 734, 677, 640, 609; HRMS-ESI $(\mathrm{m} / \mathrm{z})$ calcd for $\mathrm{C}_{13} \mathrm{H}_{10} \mathrm{BrN}_{2}\left([\mathrm{M}+\mathrm{H}]^{+}\right)$: 273.0022. Found: 273.0023 .

\section{Experiment for the Synthesis of Compound 5 (Scheme 6).}

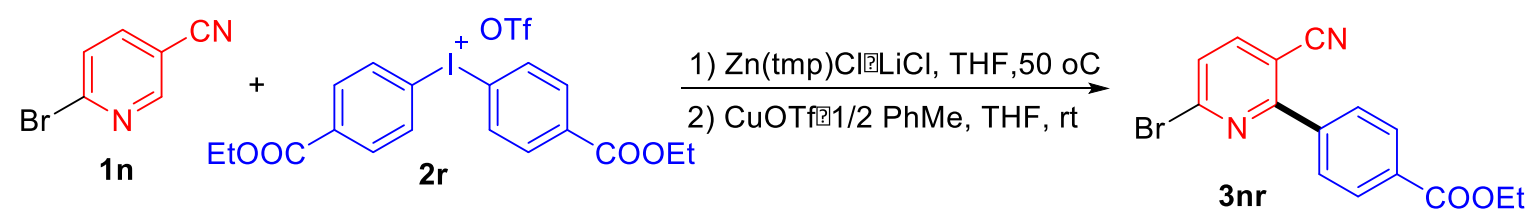

To an oven-dried Schlenk tube was added $1 \mathrm{n}(54.6 \mathrm{mg}, 0.3 \mathrm{mmol}, 1.0$ equiv) and the solution of $\mathrm{Zn}(\mathrm{tmp}) \mathrm{Cl} \cdot \mathrm{LiCl}\left(0.6 \mathrm{mmol}, 2.0\right.$ equiv) in THF at $50{ }^{\circ} \mathrm{C}$ under $\mathrm{N}_{2}$. The mixture was stirred for $1 \mathrm{~h}$ and then was cooled down to room temperature. Then iodonium salt 2r (344.3 mg, $0.6 \mathrm{mmol}, 2.0$ equiv), CuOTf• $1 / 2 \mathrm{PhMe}$ (7.8 mg, $0.03 \mathrm{mmol}, 10 \mathrm{~mol} \%$ ) and the fresh distilled THF $(3.0 \mathrm{~mL})$ were added under $\mathrm{N}_{2}$. The reaction was stirred for 8 $\mathrm{h}$ at room temperature and then was quenched by the addition of $\mathrm{MeOH}(1 \mathrm{~mL})$. The mixture was filtered though a pale of $\mathrm{Al}_{2} \mathrm{O}_{3}$ and washed with $\mathrm{Et}_{2} \mathrm{O}(5 \mathrm{~mL} \times 3)$ and 
$\mathrm{CH}_{2} \mathrm{Cl}_{2}(5 \mathrm{~mL} \times 3)$. The combined mixtures were concentrated in vacuo. The crude product was purified by silica column chromatography $(10 \% \rightarrow 20 \%$ EtOAc in hexanes) to afford $3 \mathrm{nr}$ as a white solid (56.1 $\mathrm{mg}, 57 \%$ yield). $\mathrm{R}_{f}=0.28$ (20\% EtOAc in hexanes); ${ }^{1} \mathrm{H}$ NMR $\left(400 \mathrm{MHz}, \mathrm{CDCl}_{3}\right) \delta 8.17(\mathrm{~d}, J=8.4 \mathrm{~Hz}, 2 \mathrm{H}), 8.00(\mathrm{~d}, J=8.4 \mathrm{~Hz}, 2 \mathrm{H}), 7.89$ (d, $J=8.4 \mathrm{~Hz}, 1 \mathrm{H}), 7.59$ (d, $J=8.4 \mathrm{~Hz}, 1 \mathrm{H}), 4.40$ (q, $J=7.2 \mathrm{~Hz}, 2 \mathrm{H}), 1.40(\mathrm{t}, J=7.2 \mathrm{~Hz}$, $3 \mathrm{H}) ;{ }^{13} \mathrm{C}$ NMR $\left(100 \mathrm{MHz}, \mathrm{CDCl}_{3}\right) \delta 165.7,160.5,145.8,143.1,139.3,132.3,129.8$, 128.9, 126.9, 116.6, 106.5, 61.2, 14.2. IR (film): 2228, 1713, 1575, 1560, 1540, 1428, 1406, 1363, 1297, 1220, 1205, 1185, 1080, 1046, 1017, 859, 831, 784, 724, 698, 651; HRMS-ESI (m/z) calcd for $\mathrm{C}_{15} \mathrm{H}_{12} \mathrm{BrN}_{2} \mathrm{O}_{2}\left([\mathrm{M}+\mathrm{H}]^{+}\right)$: 331.0077 . Found: 331.0076 .

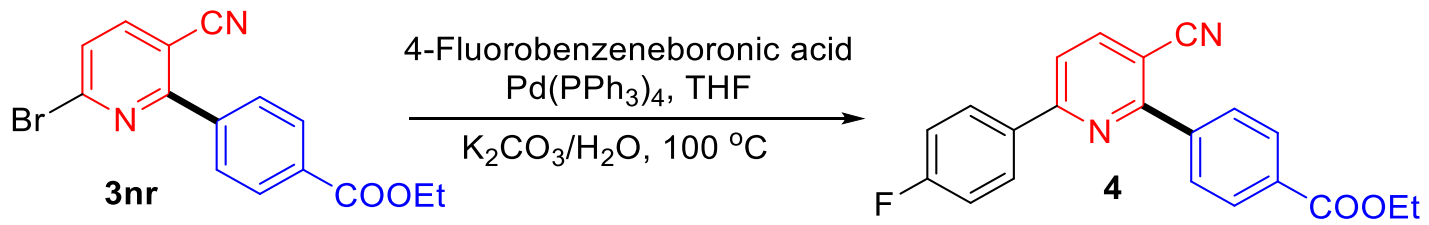

To an nitrogen-filled round-bottom flask was added $3 \mathbf{n r}$ ( $49.5 \mathrm{mg}, 0.15 \mathrm{mmol}, 1.0$ equiv), $\mathrm{Pd}\left(\mathrm{PPh}_{3}\right)_{4}$ (17.3 mg, $0.015 \mathrm{mmol}, 0.1$ equiv.), $\mathrm{K}_{2} \mathrm{CO}_{3}(41.4 \mathrm{mg}, 0.3 \mathrm{mmol}, 2.0$ equiv.) and a solution of 4-fluorobenzeneboronic acid ( $25.2 \mathrm{mg}, 0.18 \mathrm{mmol}, 1.2$ equiv) in THF (4 mL) followed by $\mathrm{H}_{2} \mathrm{O}(1 \mathrm{~mL})$. The reaction mixture was heated to $100{ }^{\circ} \mathrm{C}$ and stirred for $4 \mathrm{~h}$. The reaction mixture was cooled down to room temperature, quenched with water $(5 \mathrm{~mL})$, and extracted with EtOAc $(5 \mathrm{~mL} \times 3)$. The combined organic layers were washed with brine, dried over $\mathrm{Na}_{2} \mathrm{SO}_{4}$, and filtered. The solvent was removed under reduced pressure. The residue was purified by silica column chromatography $(10 \% \rightarrow 20 \%$ EtOAc in hexanes) afforded 4 as a white solid (50.7 $\mathrm{mg}, 98 \%$ yield). $\mathrm{R}_{f}=0.18(12.5 \%$ EtOAc in hexanes); ${ }^{1} \mathrm{H}$ NMR (400 MHz, $\left.\mathrm{CDCl}_{3}\right) \delta 8.20(\mathrm{~d}, J=8.4 \mathrm{~Hz}, 2 \mathrm{H}), 8.14-8.07(\mathrm{~m}, 5 \mathrm{H})$, $7.77(\mathrm{~d}, J=8.4 \mathrm{~Hz}, 1 \mathrm{H}), 7.18$ (t, $J=8.4 \mathrm{~Hz}, 2 \mathrm{H}), 4.41(\mathrm{q}, J=7.1 \mathrm{~Hz}, 2 \mathrm{H}), 1.41$ (t, $J=7.1$ $\mathrm{Hz}, 3 \mathrm{H}) ;{ }^{13} \mathrm{C} \mathrm{NMR}\left(100 \mathrm{MHz}, \mathrm{CDCl}_{3}\right) \delta 165.9,164.3(\mathrm{~d}, J=253.2 \mathrm{~Hz}), 159.3,158.5$, 142.5, 141.1, $133.2(\mathrm{~d}, J=3.1 \mathrm{~Hz}), 131.7,129.7,129.4$ (d, $J=8.7 \mathrm{~Hz}), 128.9,117.8$, 117.6, $115.9(\mathrm{~d}, J=21.6 \mathrm{~Hz}),, 105.2,61.2,14.2 ;{ }^{19} \mathrm{~F}$ NMR $\left(376 \mathrm{MHz}, \mathrm{CDCl}_{3}\right) \delta-109.58$ to -109.65 (m). IR (film): 2219, 1712, 1567, 1554, 1506, 1449, 1360, 1234, 1203, 1185 , 1153, 1126, 1102, 1057, 1017, 856, 824, 807, 787, 761, 719, 699, 671; HRMS-ESI (m/z) calcd for $\mathrm{C}_{21} \mathrm{H}_{16} \mathrm{FN}_{2} \mathrm{O}_{2}\left([\mathrm{M}+\mathrm{H}]^{+}\right): 347.1190$. Found:347.1196. 


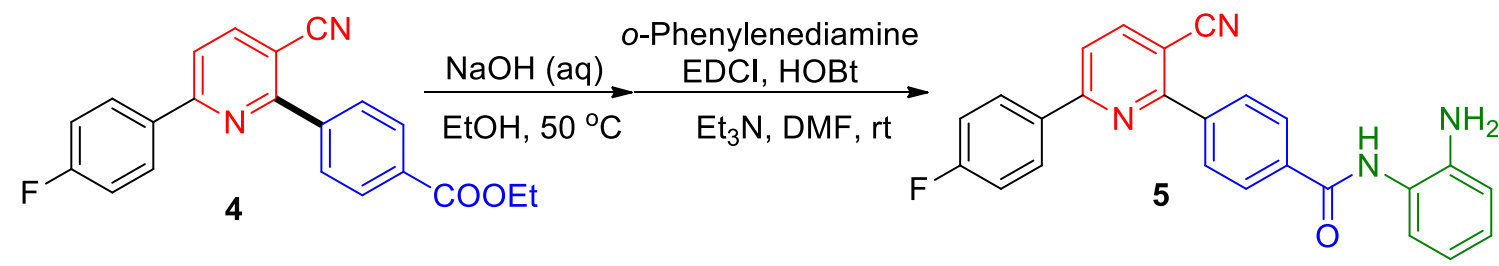

To a solution of compound 4 ( $21.0 \mathrm{mg}, 0.06 \mathrm{mmol}, 1.0$ equiv) in $\mathrm{EtOH} \mathrm{(} 3 \mathrm{~mL})$ was added an aqueous solution of $\mathrm{NaOH}(2 \mathrm{~N}, 0.4 \mathrm{~mL})$ and then the resulting mixture was stirred at $50{ }^{\circ} \mathrm{C}$ overnight. The reaction mixture was cooled down, diluted with water $(10 \mathrm{~mL})$, and acidified with the aqueous solution of $\mathrm{HCl}(3 \mathrm{~N})$ to $\mathrm{pH} 2 \sim 4$. The corresponding mixture was extracted with ethyl acetate $(5 \mathrm{~mL} \times 5)$. The combined organic layers were dried over anhydrous $\mathrm{Na}_{2} \mathrm{SO}_{4}$, then the solvent was evaporated in vacuo. The residue was used directly for next step without further purification.

To a solution of the above crude mixture in DMF (2 mL) was added 1-ethyl-3-(3 dimethyllaminopropyl) carbodiimide hydrochloride (23.0 mg, $0.12 \mathrm{mmol}, 2.0$ equiv), hydroxybenzotriazole (12.2 $\mathrm{mg}, 0.09 \mathrm{mmol}, 1.5$ equiv), triethylamine $(33.3 \mathrm{ml}, 0.24$ mmol, 4.0 equiv) and $o$-phenylenediamine ( $13.0 \mathrm{mg}, 0.12 \mathrm{mmol}, 2.0$ equiv). The mixture was stirred for $20 \mathrm{~h}$ at room temperature. The mixture was diluted with brine $(10 \mathrm{~mL})$, and extracted with EtOAc $(5 \mathrm{~mL} \times 3)$. The combined organic layers were washed with brine, dried over $\mathrm{Na}_{2} \mathrm{SO}_{4}$, and filtered. The solvent was removed under reduced pressure. The residue was purified by silica column chromatography $(30 \% \rightarrow 50 \%$ EtOAc in hexanes) to afford 5 as a light yellow solid ( $21.7 \mathrm{mg}, 89 \%$ yield). $\mathrm{R}_{f}=0.25$ (50\% EtOAc in hexanes); ${ }^{1} \mathrm{H}$ NMR (400 MHz, $\left.\mathrm{CDCl}_{3}\right) \delta 8.18-8.12(\mathrm{~m}, 5 \mathrm{H}), 8.08(\mathrm{~d}, J=8.0 \mathrm{~Hz}, 2 \mathrm{H})$, $8.04(\mathrm{~s}, 1 \mathrm{H}), 7.81(\mathrm{~d}, J=8.0 \mathrm{~Hz}, 1 \mathrm{H}), 7.39(\mathrm{~d}, J=8.4 \mathrm{~Hz}, 1 \mathrm{H}), 7.22(\mathrm{t}, J=8.8 \mathrm{~Hz}, 2 \mathrm{H})$, $7.12(\mathrm{t}, J=7.2 \mathrm{~Hz}, 1 \mathrm{H}), 6.89-6.86(\mathrm{~m}, 2 \mathrm{H}), 3.90(\mathrm{~s}, 2 \mathrm{H}) ;{ }^{13} \mathrm{C} \mathrm{NMR}\left(100 \mathrm{MHz}, \mathrm{CDCl}_{3}\right) \delta$ $165.1,164.3$ (d, $J=253.2 \mathrm{~Hz}), 159.4,158.8,142.6,140.5$ (d, $J=4.1 \mathrm{~Hz}), 135.6,133.30$ (d, $J=3.2 \mathrm{~Hz}), 129.6,129.5,129.4,127.6,127.3,125.1,124.5,119.9,118.5,118.0$, 116.1 (d, $J=21.7 \mathrm{~Hz}$ ), 105.3; IR (film): 3270, 2920, 2221, 1622, 1599, 1579, 1556, 1524, 1504, 1444, 1418, 1376, 1299, 1230, 1155, 1099, 1057, 1013, 908, 854, 808, 746, 719, 700, 667; HRMS-ESI (m/z) calcd for $\mathrm{C}_{25} \mathrm{H}_{18} \mathrm{FN}_{4} \mathrm{O}\left([\mathrm{M}+\mathrm{H}]^{+}\right)$:409.1459. Found: 409.1458 . 


\section{References.}

(1) (a) Bielawski, M.; Olofsson, B. Chem. Commun. 2007, 2521. (b) Bielawski, M.; Zhu, M.; Olofsson, B. Adv. Synth. Catal. 2007, 349, 2610. (c) Phipps, R. J.; Gaunt, M. J. Science 2009, 323, 1593.

(2) (a) Ochiai, M.; Toyonari, M.; Nagaoka, T.; Chen, D.-W. Kida, M. Tetrahedron Lett. 1997, 38, 67099; (b) Skucas, E.; MacMillan, D. W. C. J. Am. Chem. Soc., 2012, 134, 9090.

(3) Tanaka, K.; Takeishi, K. Synthesis, 2007, 18, 2920.

(4) Ghosh, R.; Adarsh, N. N.; Sarkar, A. J. Org. Chem. 2010, 75, 5320.

(5) Wu, D.; Wang, Z-X. Org. Bio.Chem. 2014, 12, 6414.

(6) Jaric, M.; B. Haag, A.; Unsinn, A.; Karaghiosoff, K.; Knochel, P. Angew. Chem., Int. Ed. 2010, 49, 5451.

(7) Rao, M. L. N.; Dhanorkar, R. J. Eur. J. Org. Chem. 2014, 5214.

(8) Bernhardt, S.; Manolikakes, G.; Kunz, T.; P. Knochel, Angew. Chem., Int. Ed. 2011, 50, 9205 .

(9) Rao, M. L. N.; Dhanorkar, R. J. Tetrahedron, 2001, 71, 338.

(10) Abbott, L.; Li, T. Patent: WO2005/111003, 2005.

(11) Gruenenthal, G. Patent: US2007/105861, 2007.

(12) Guo, P.; Joo, J. M.; Rakshit, S.; Sames, D. J. Am. Chem. Soc. 2011, 133, 16338.

(13) Li, J.; Benard, S.; Neuville, L.; Zhu, J. Org. Lett. 2012, 14, 5980.

(14) Wu, M.; Hu, X.; Liu, J.; Liao, Y.; Deng, G.-J. Org. Lett. 2012, 14, 2722.

(15) Laskar, I. R.; Chen, T.-M. Chem. Mater. 2004, 16, 111.

(16) Liu, K. M.; Liao, L. Y.; Duan, X. F. Chem. Commun. 2015, 51, 1124.

(17) Liu, L.; Floreancig, P. E. Org. Lett. 2010, 12, 4686.

(18) Sezen, B.; Sames, D. Org. Lett. 2003, 5, 3607.

(19) Sato, N.; Narita, N. J. Heterocycl. Chem. 1999, 36, 783.

(20) Ding, S.; Jiao, N. J. Am. Chem. Soc. 2011, 133, 12374.

(21) Lee, W.-C.; Wang, T.-H.; Ong, T.-G. Chem. Commun., 2014, 50, 3671.

(22) Li, Z.; Twieg, R. J. Chem. Eur. J. 2015, 21, 15534.

(23) Combret, Y.; Torche, J.-J.; Ple, N.; Duflos, J.; Dupas, G.; Bourguignon, J.; Queguiner, G. Tetrahedron, 1991, 47, 9369. 
(24) Jens, S.; Gerhard, M. Synthesis, 1999, 1, 100.

(25) Kloss, F.; Koehn, U.; Jahn, B. O.; Hager, M. D.; Goerls, H.; Schubert, U. S. Chem. Asian J, 2011, 6, 2816. 
VII. NMR spectra

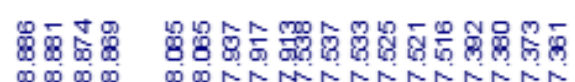

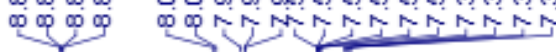

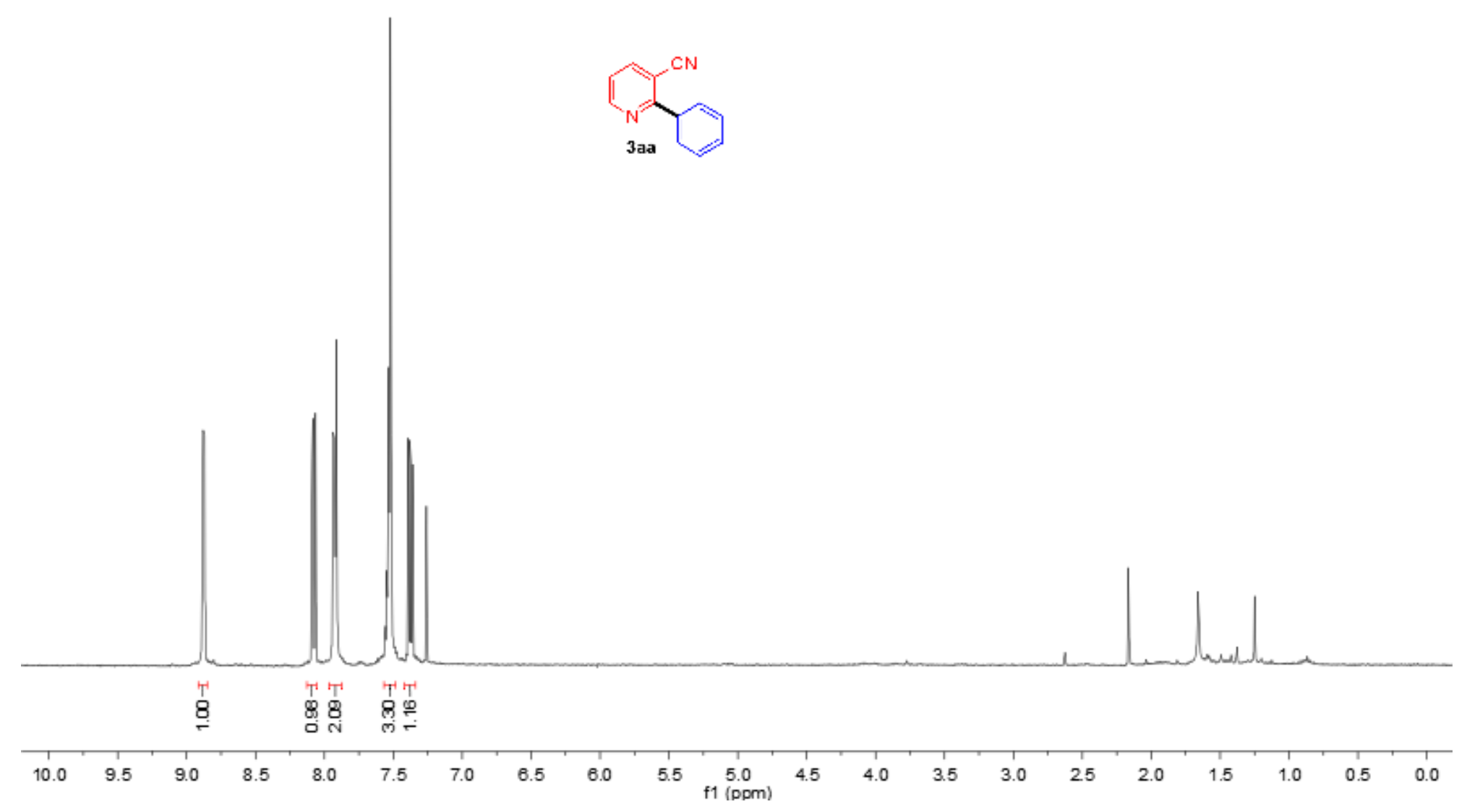




$$
=1
$$




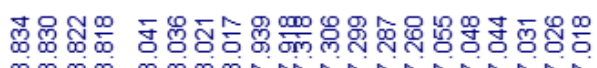

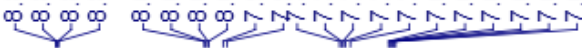
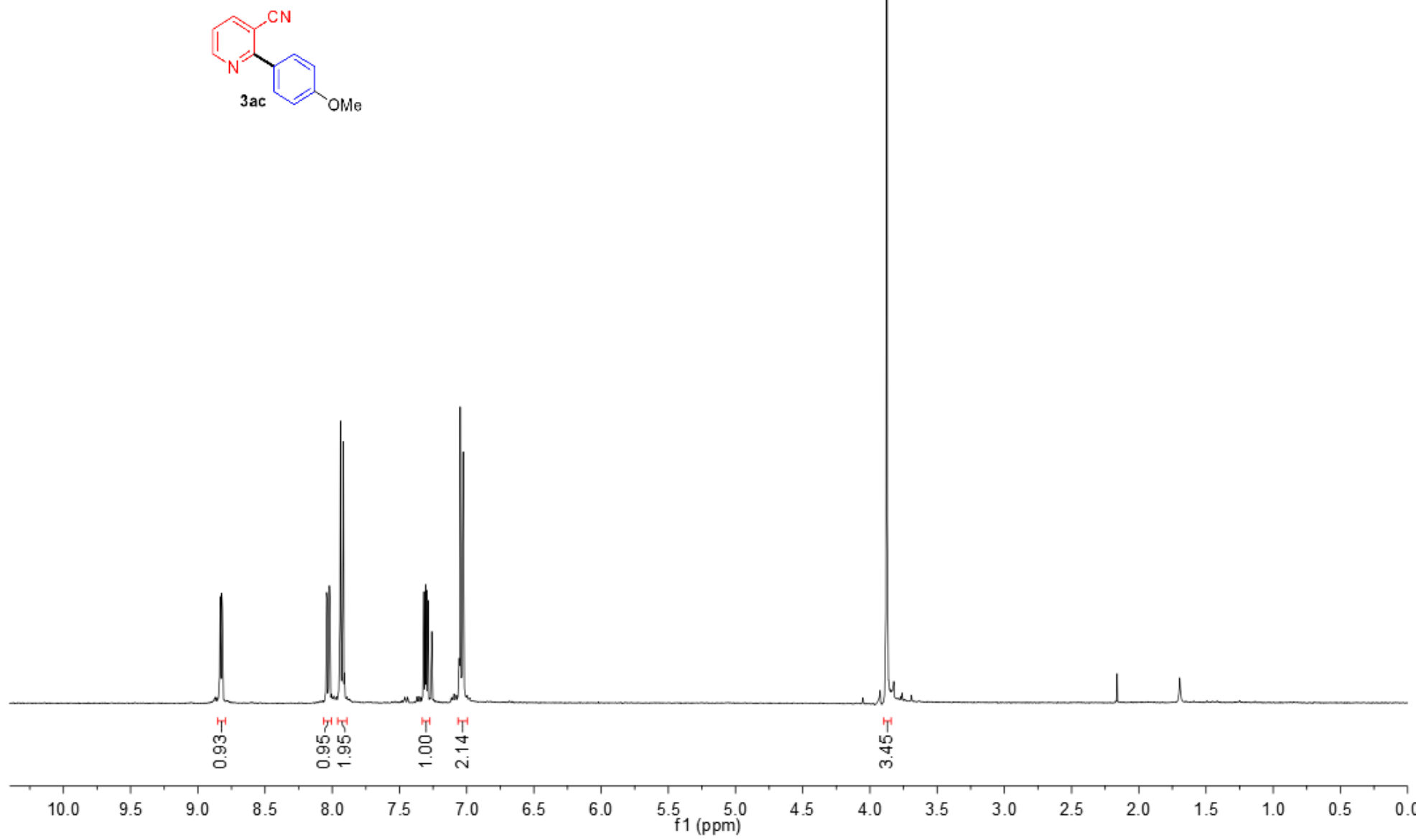


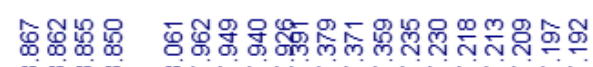

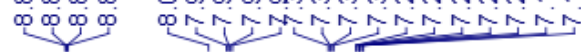

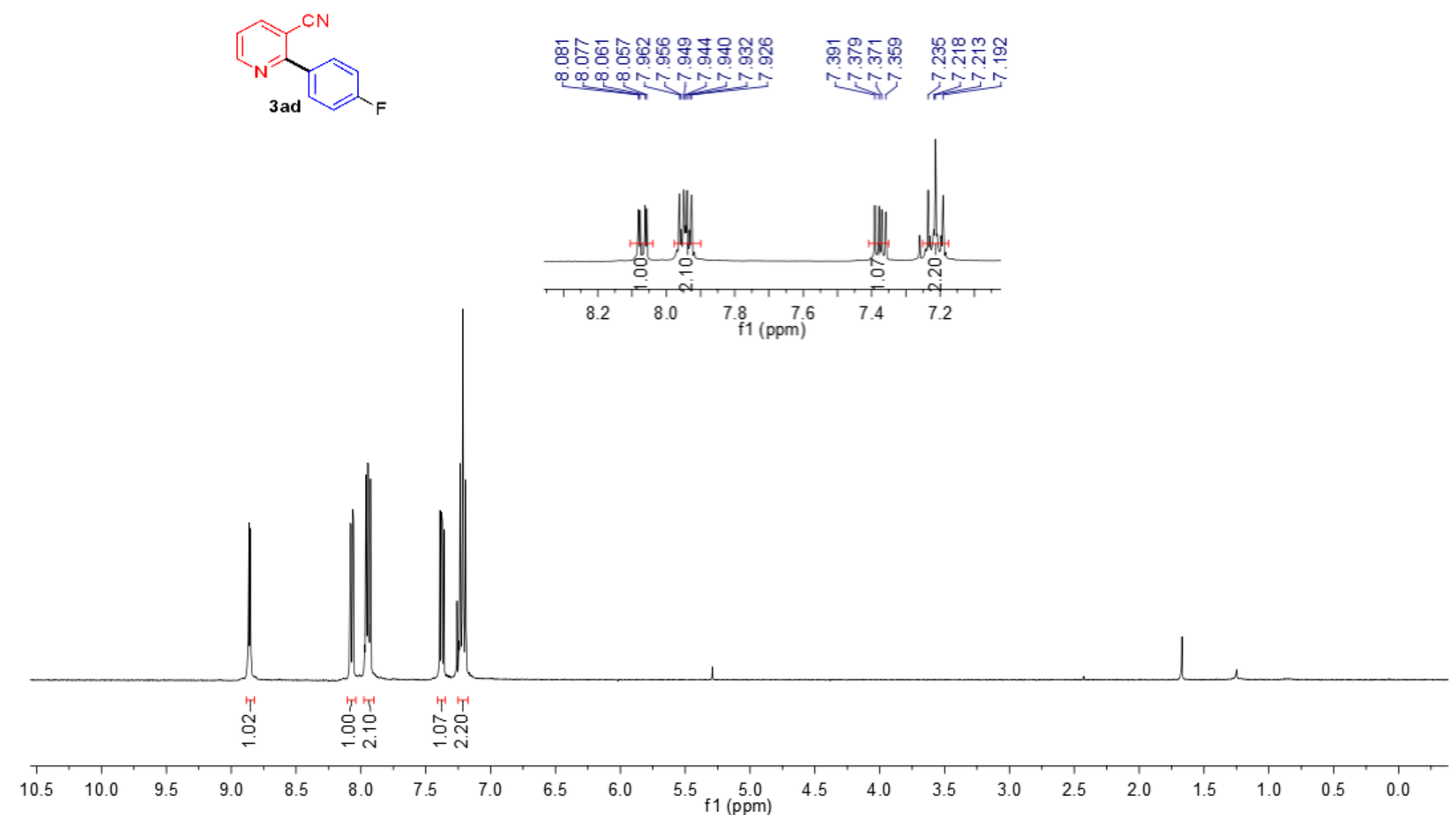




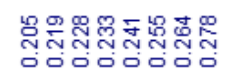

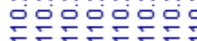

运运远
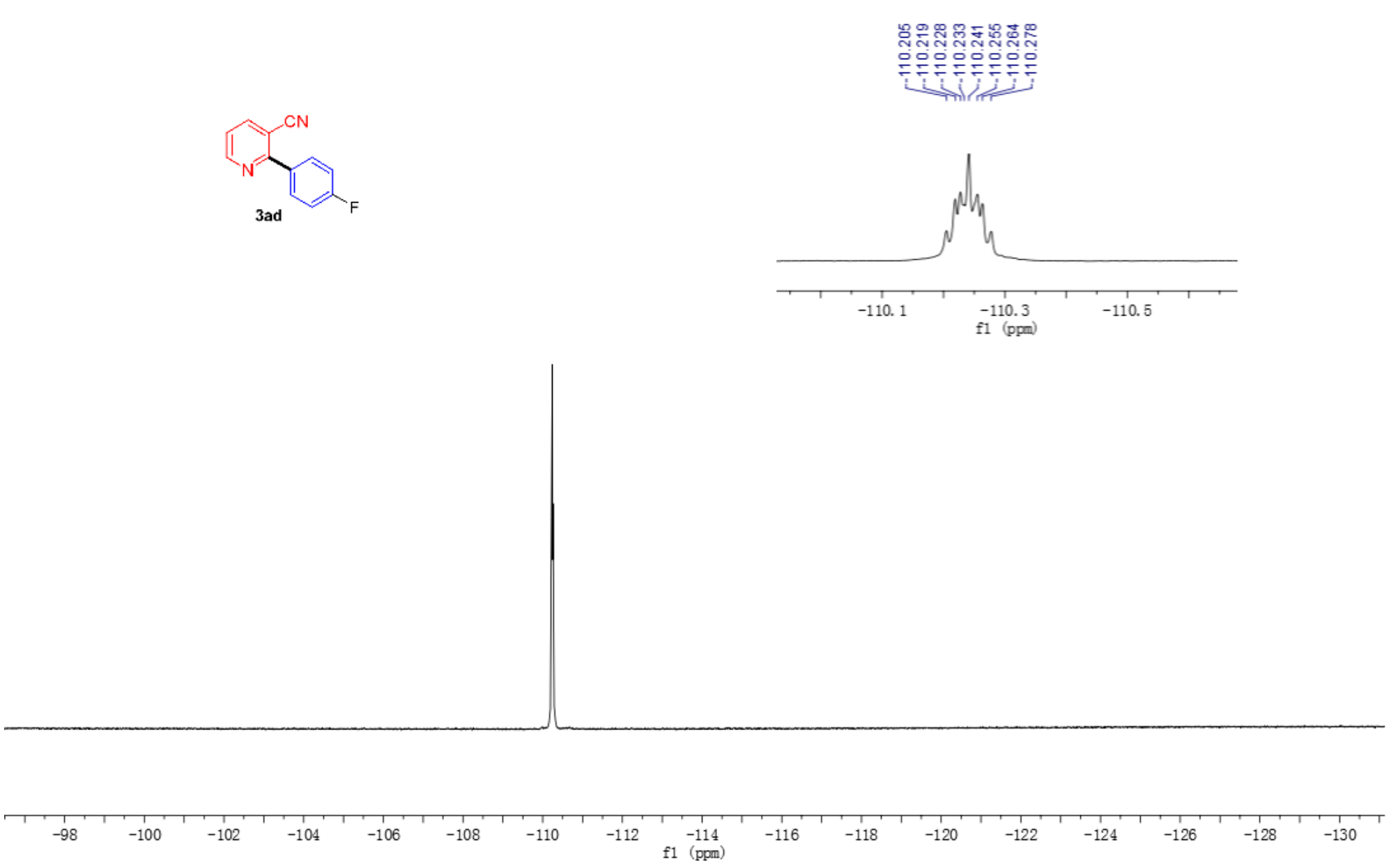


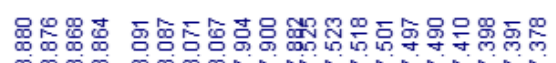

ن

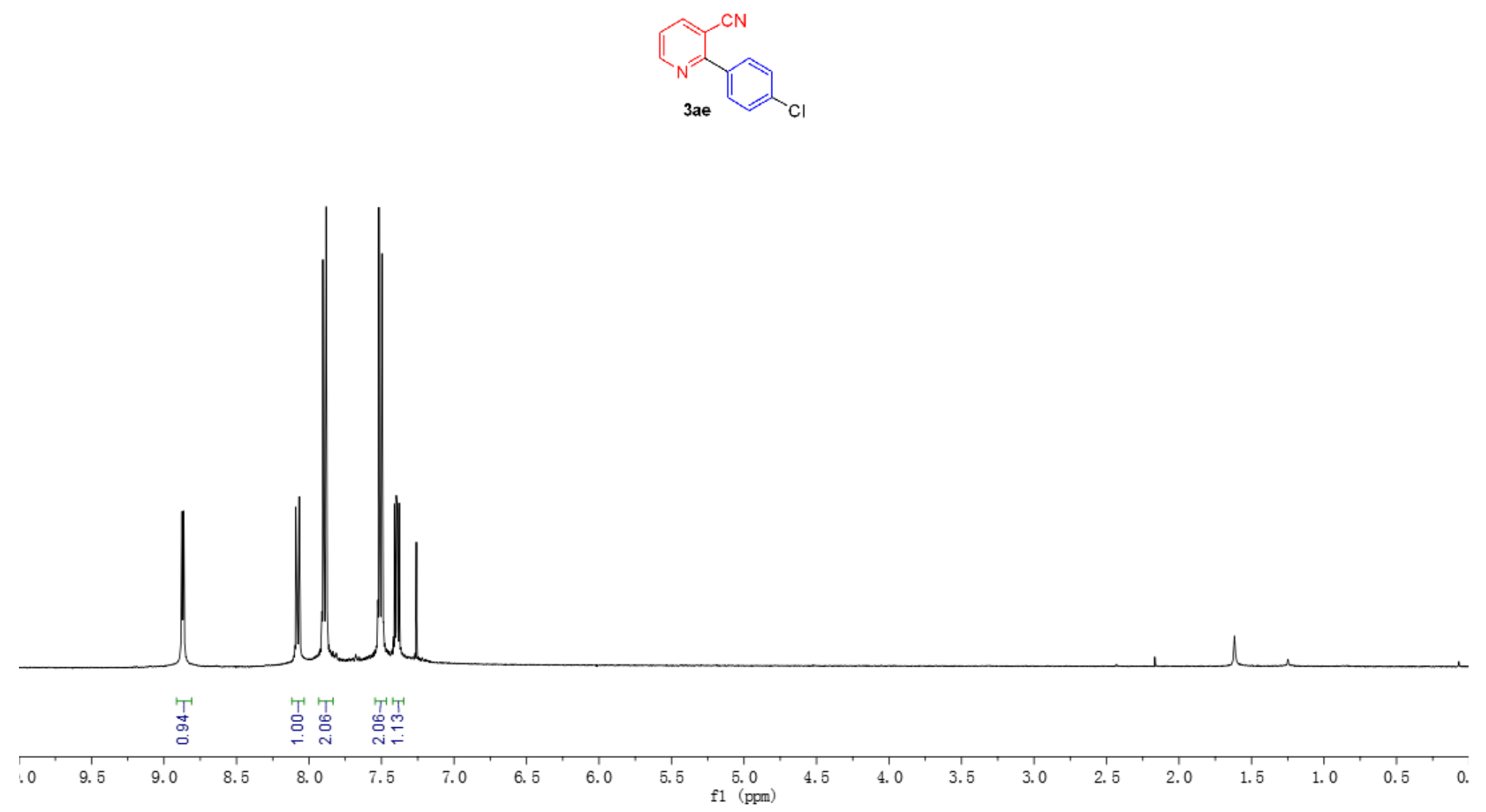



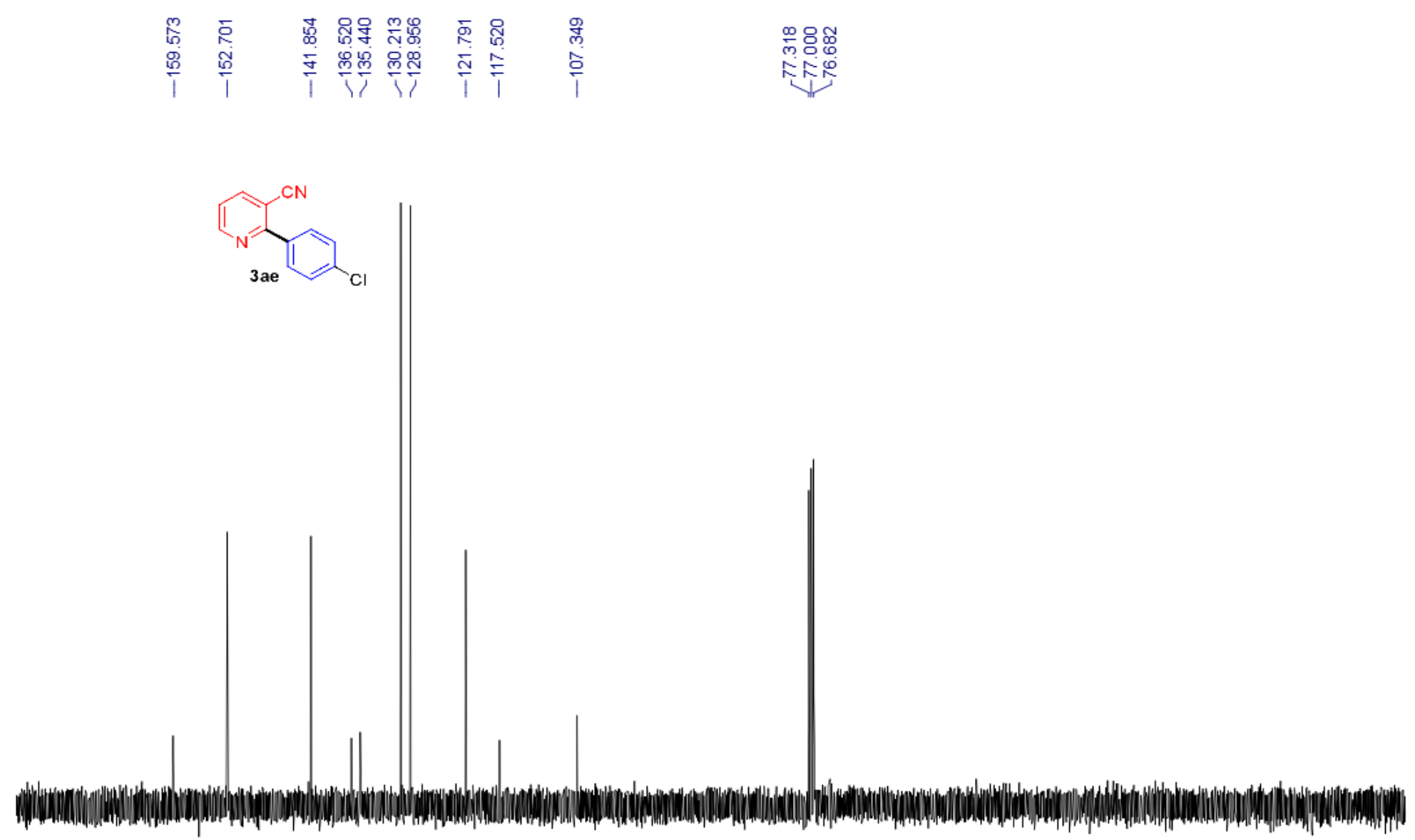


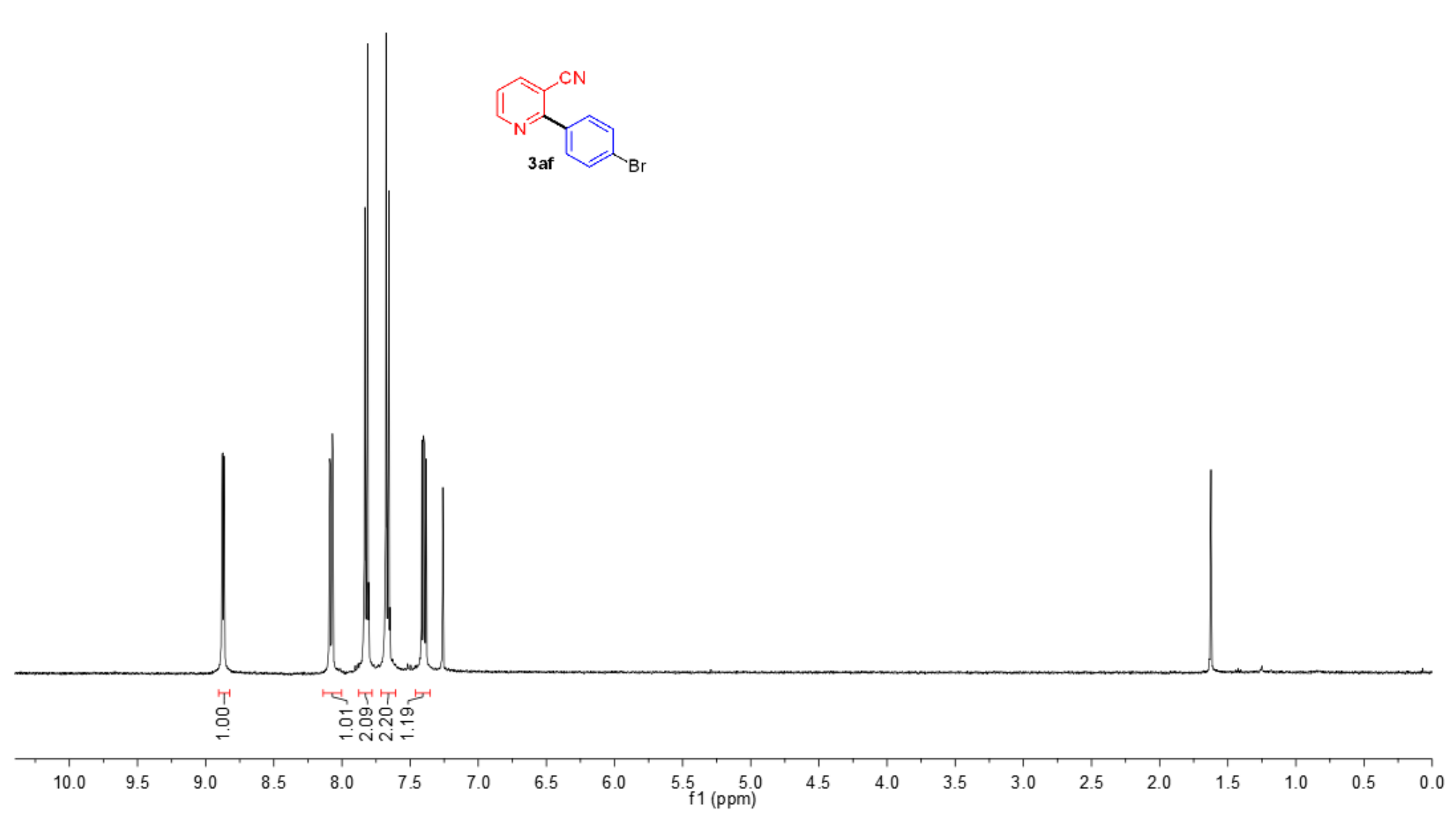



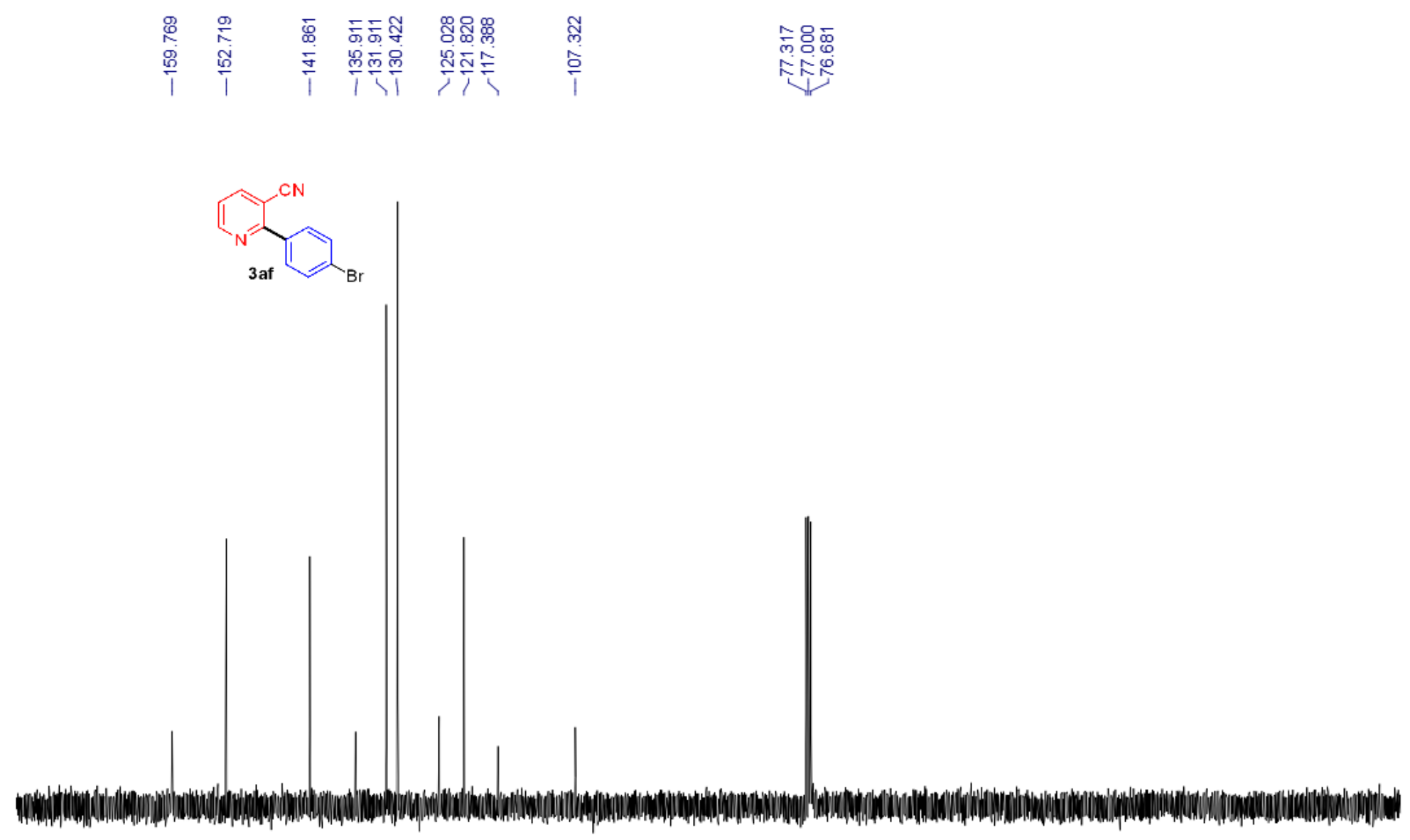

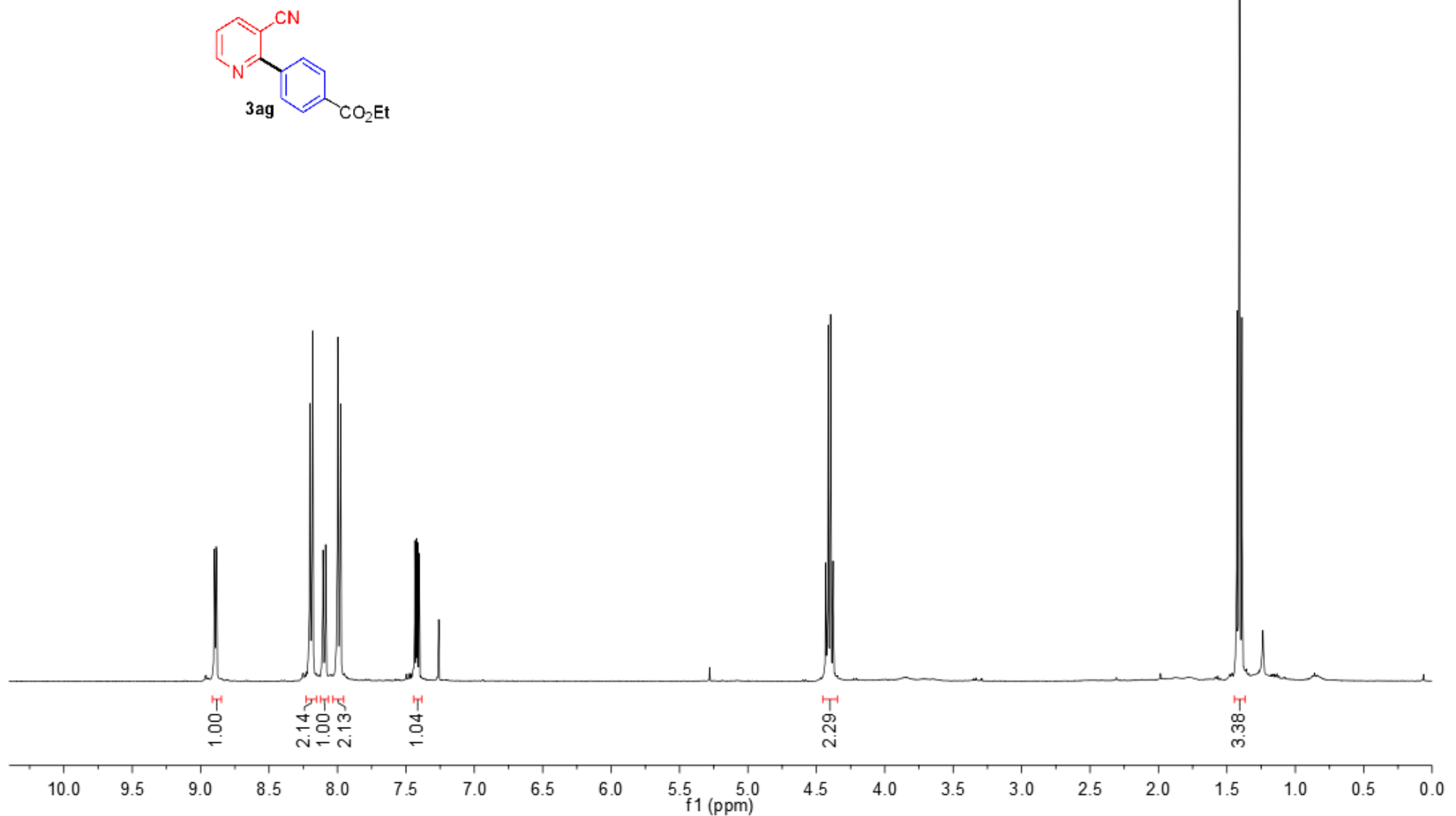


$$
\text { . }
$$




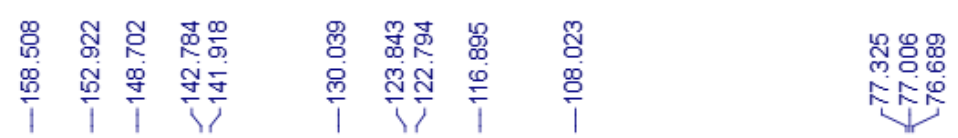
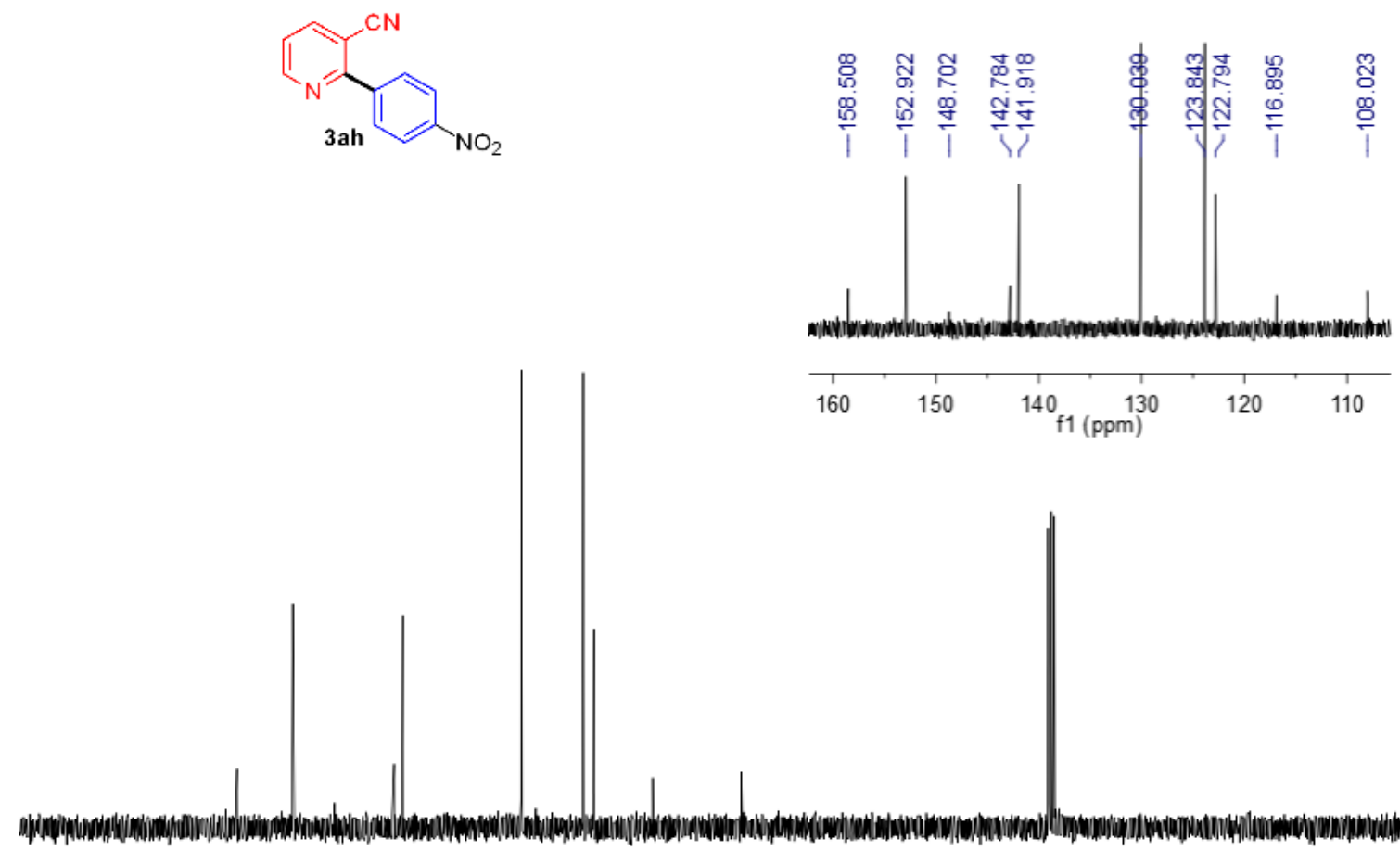

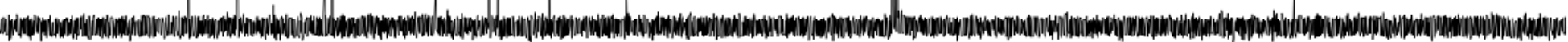




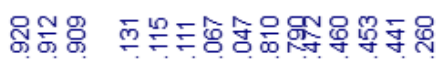

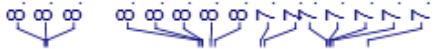
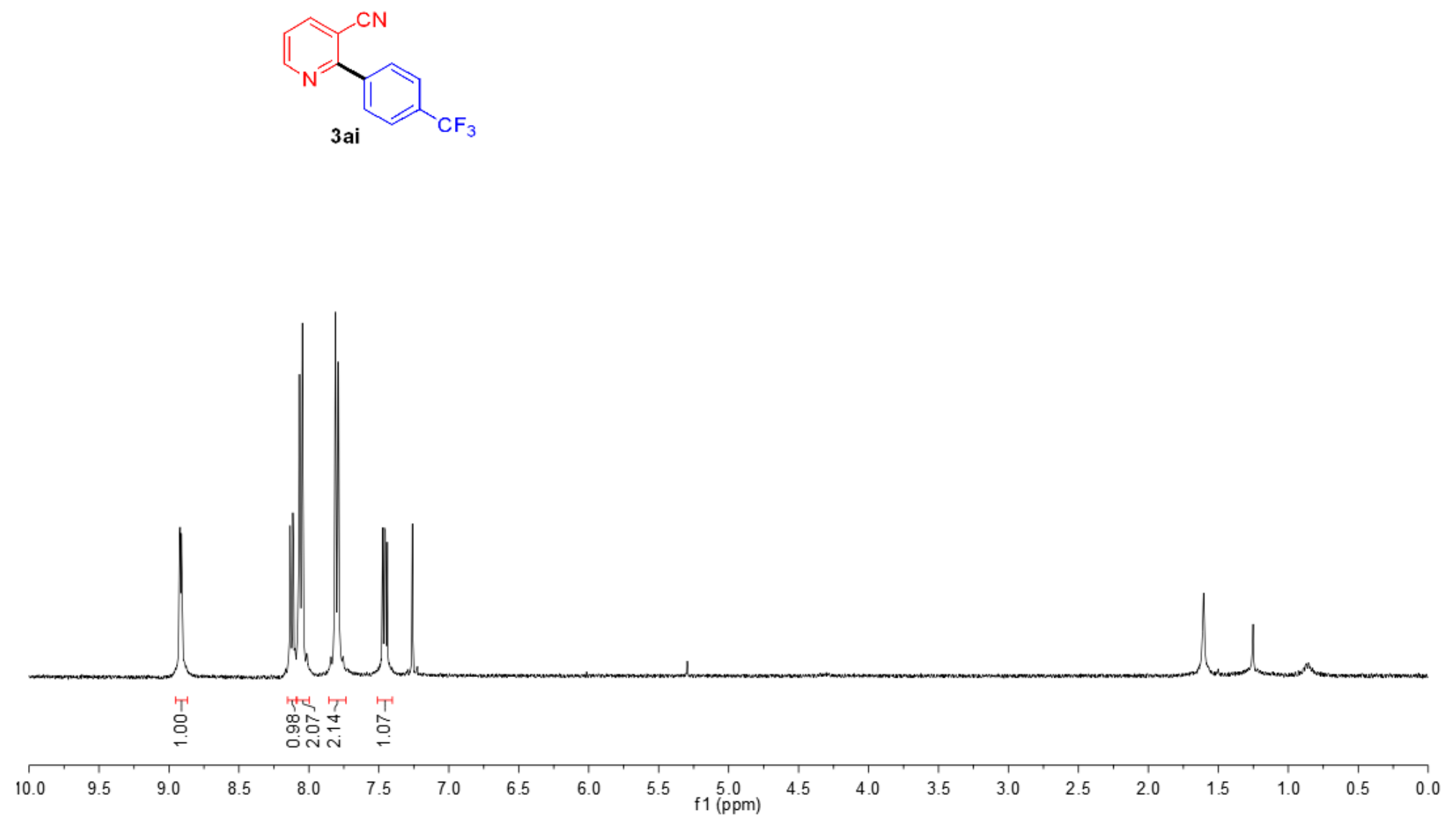


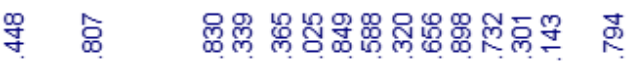

ด กั

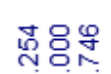

趑

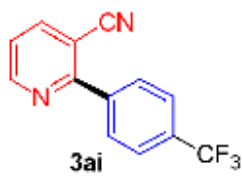

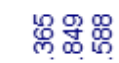

लिल흐

wh
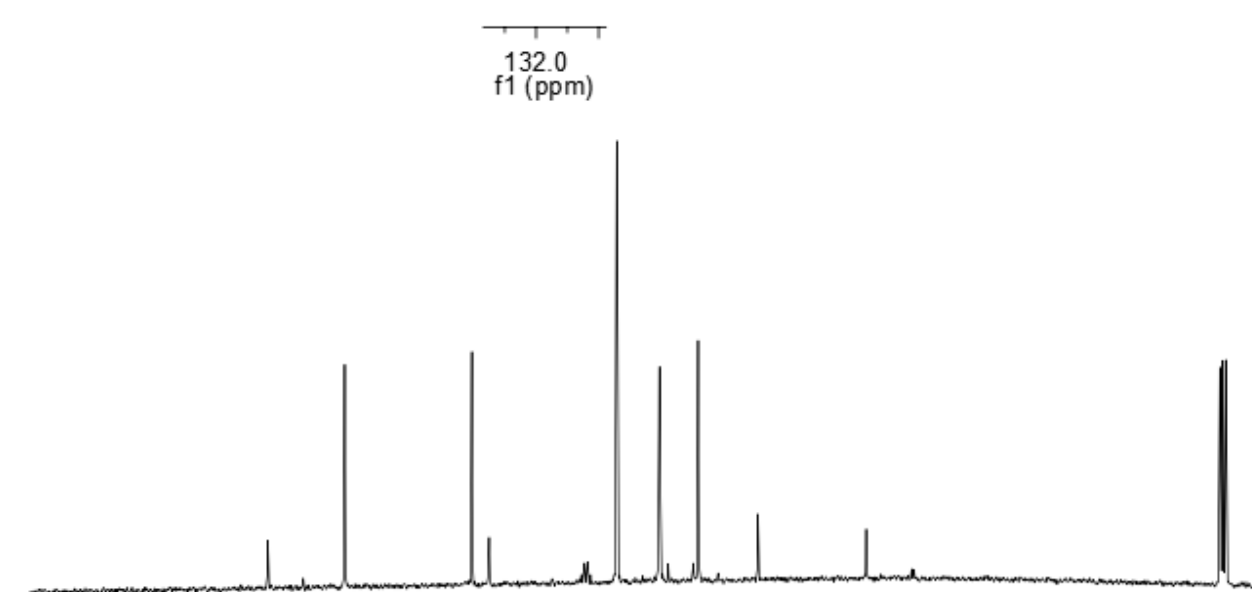

180

$170 \quad 160$

$150 \quad 140$

130

$120 \quad 110$

$100 \quad 90 \quad 80$

$70 \quad 60$

$60 \quad 50$

$\begin{array}{llll}1 & 1 & 1 & 1\end{array}$ 


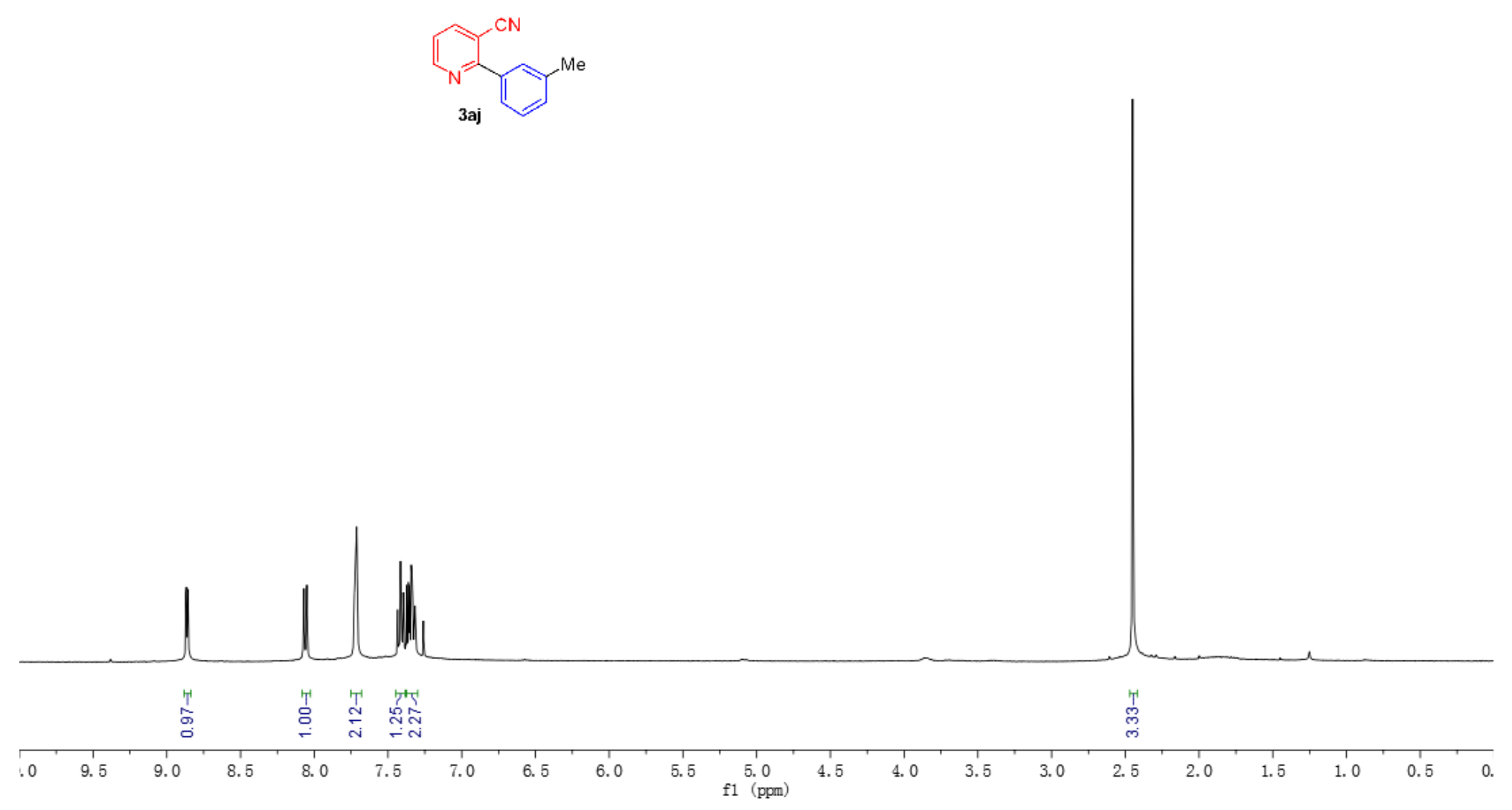




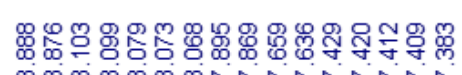

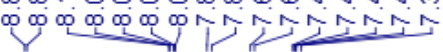
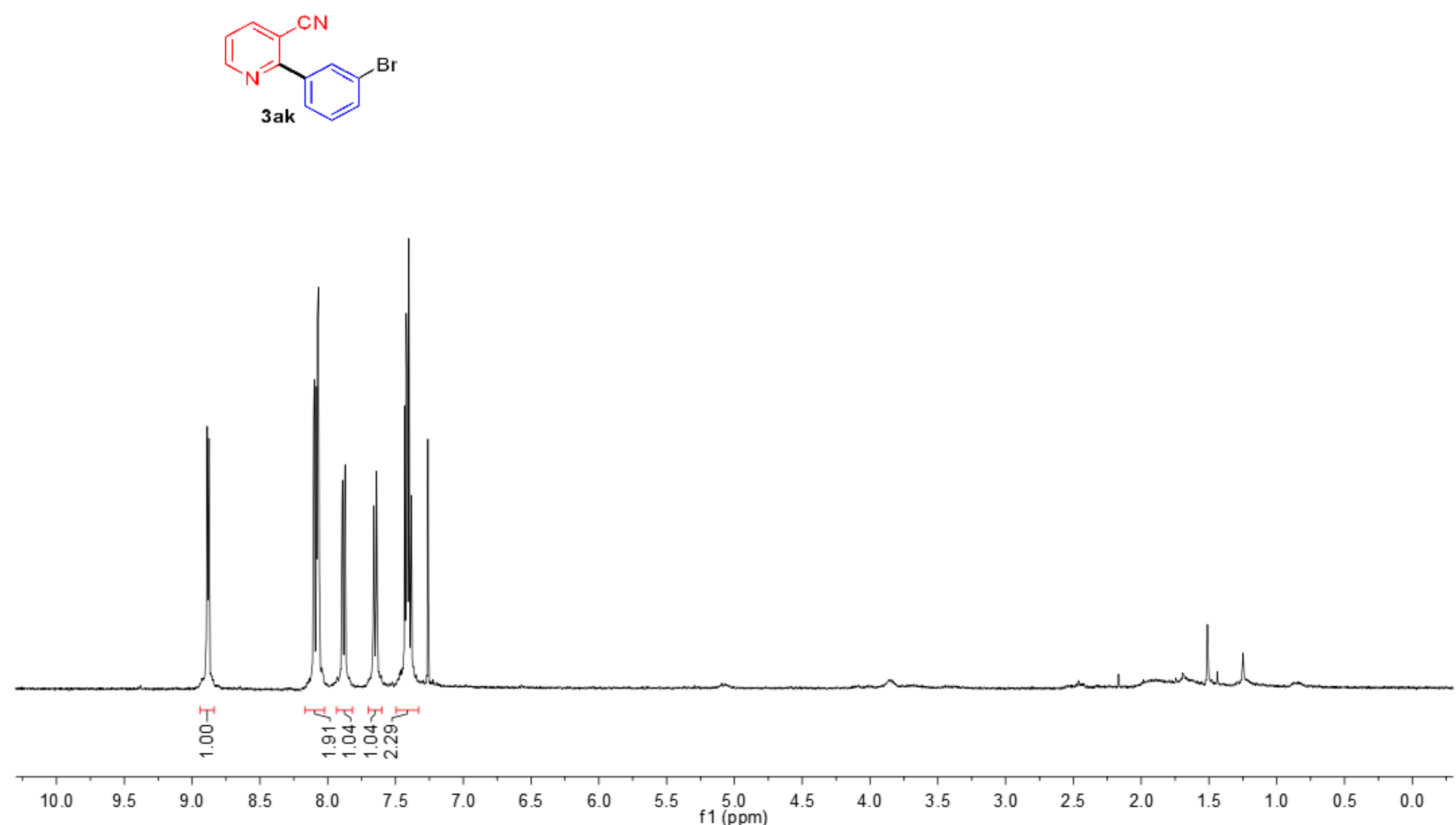


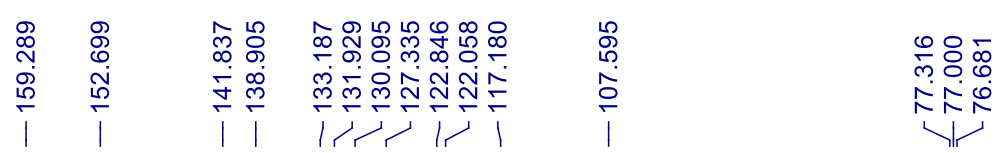

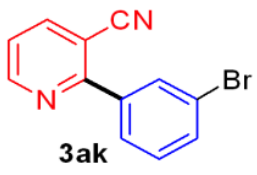

$\mid$ || 


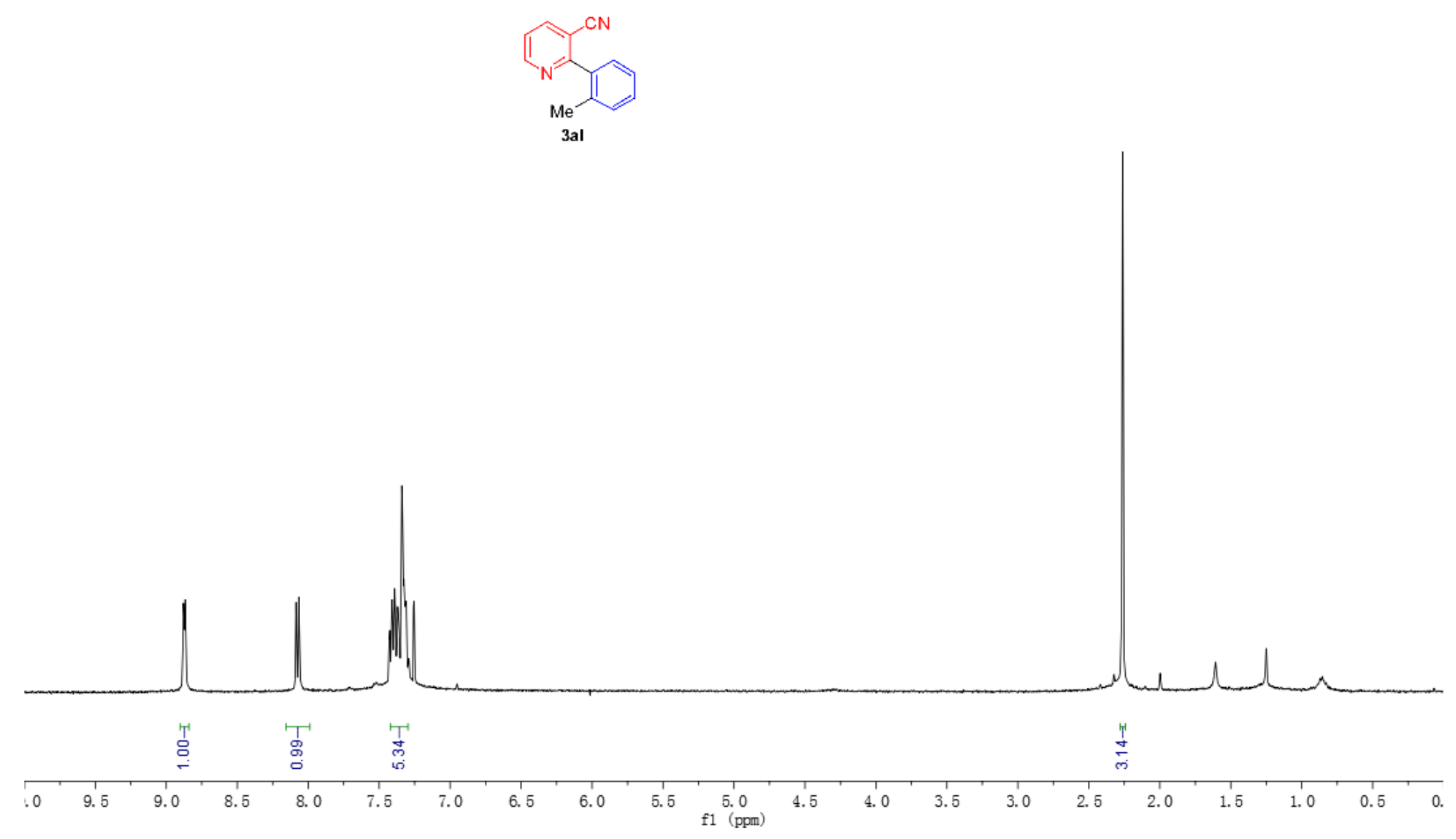




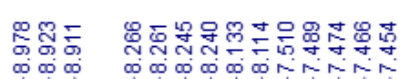

wi viu
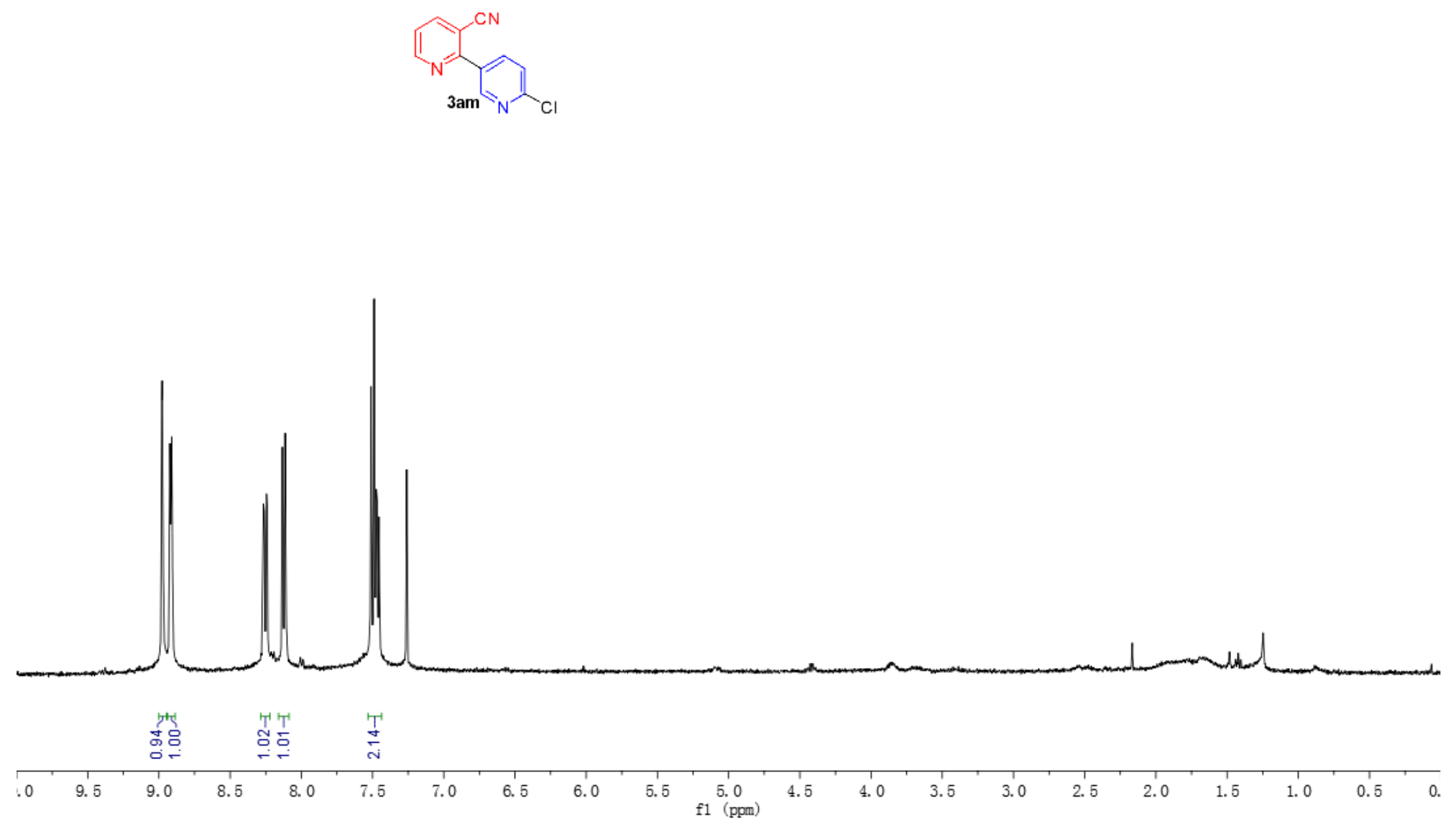

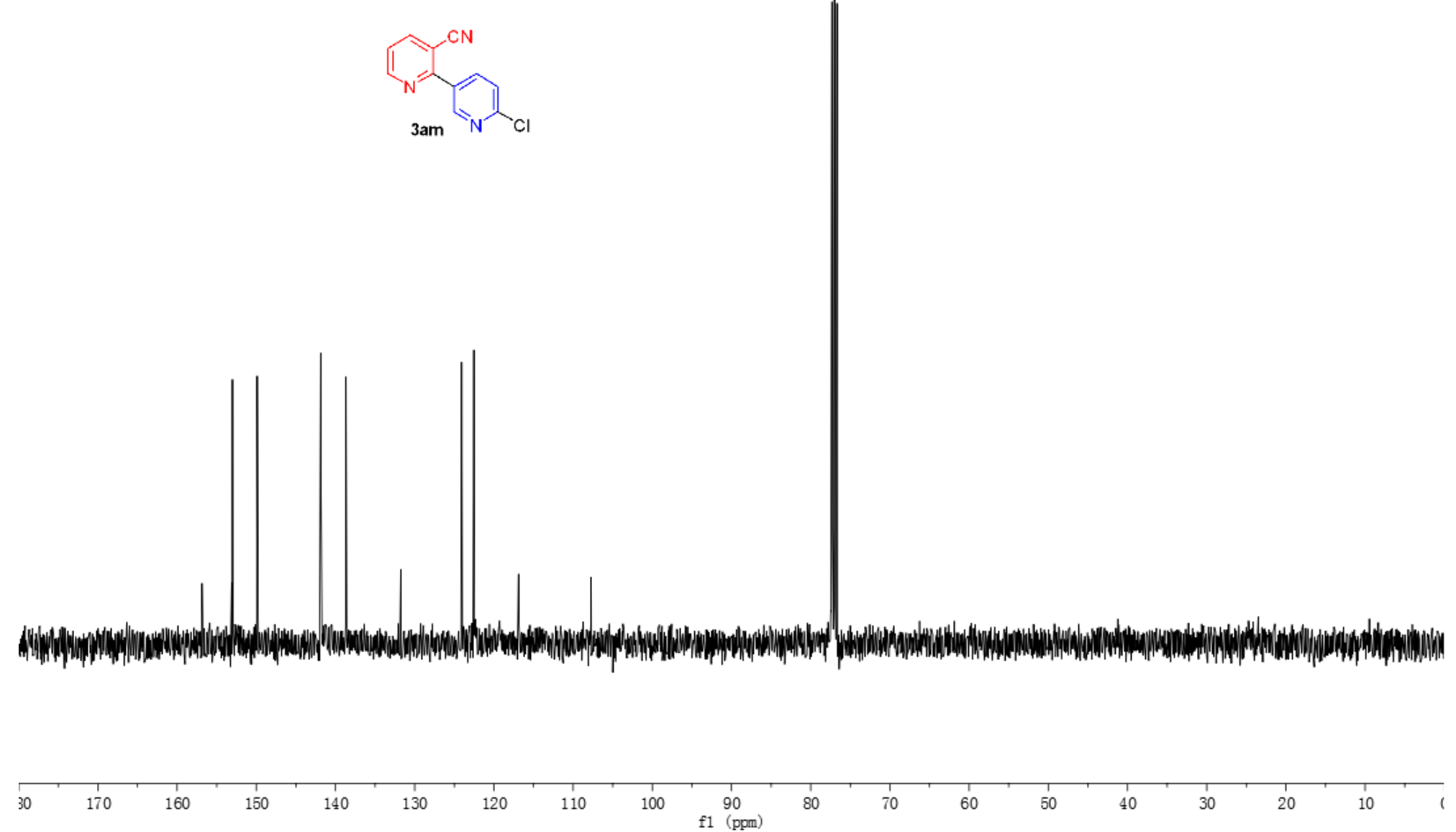


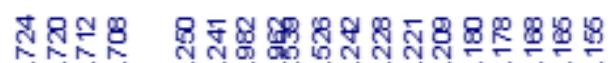

ou
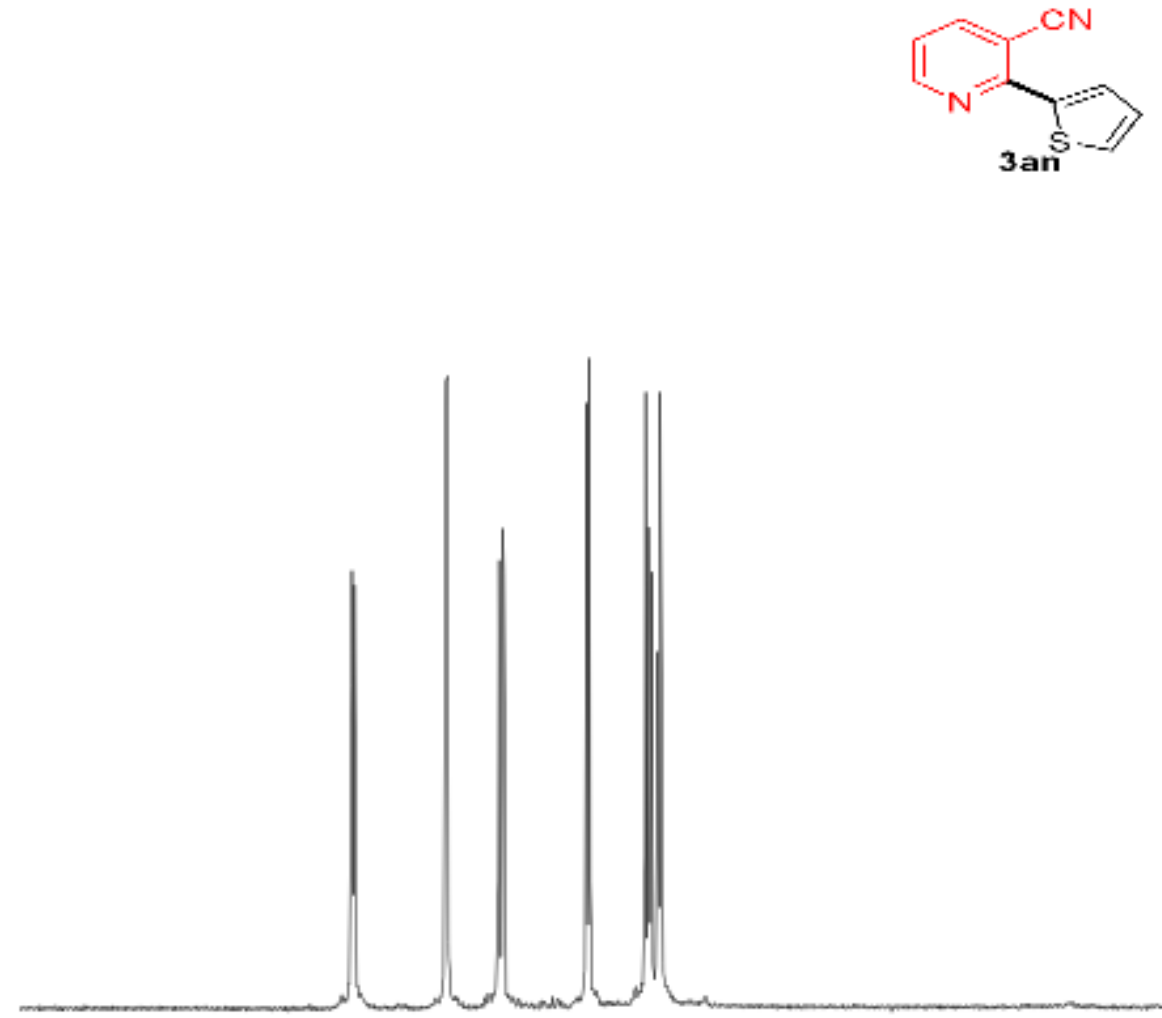


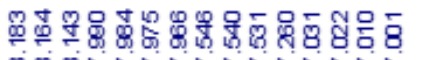

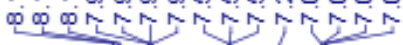
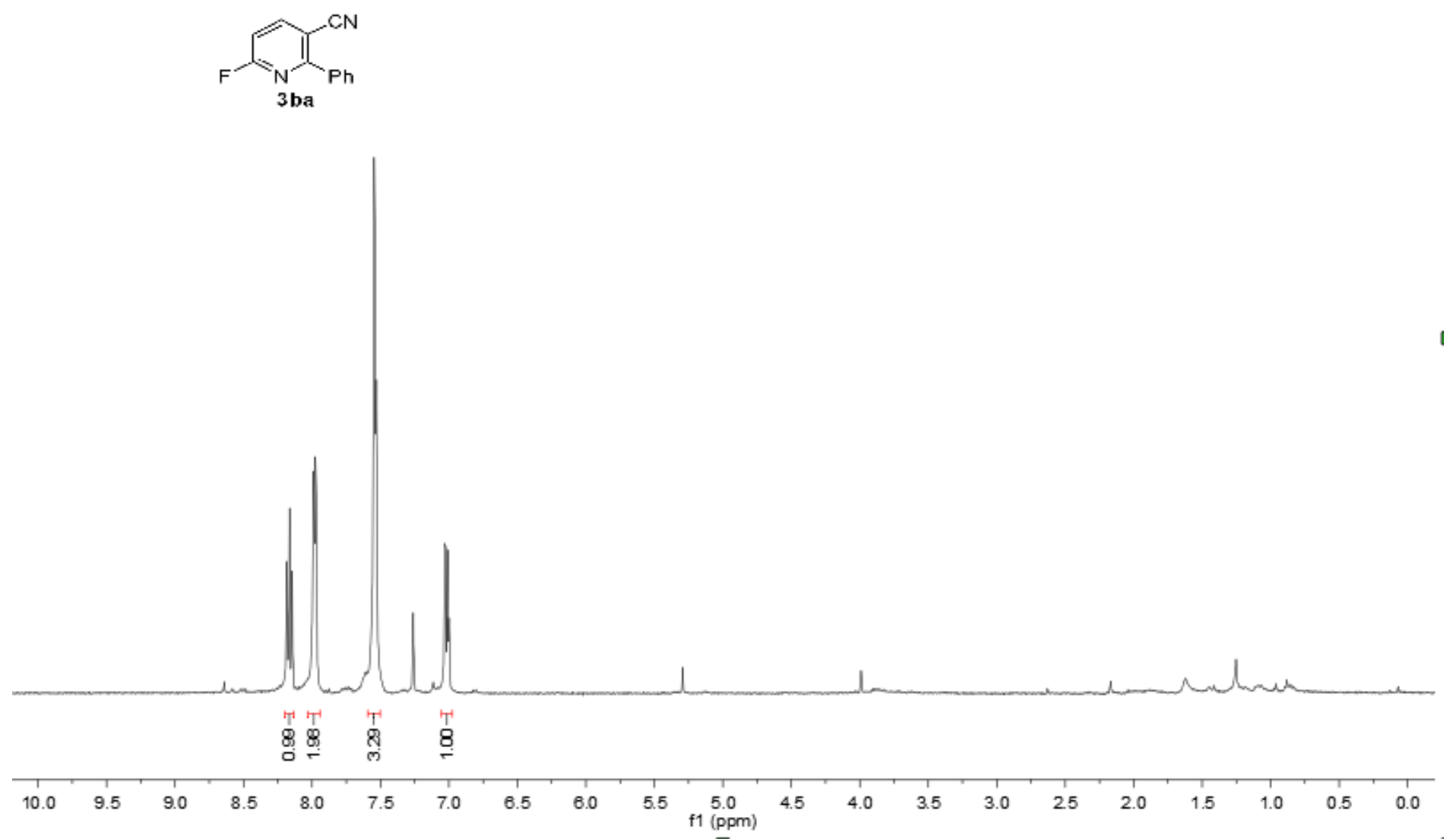


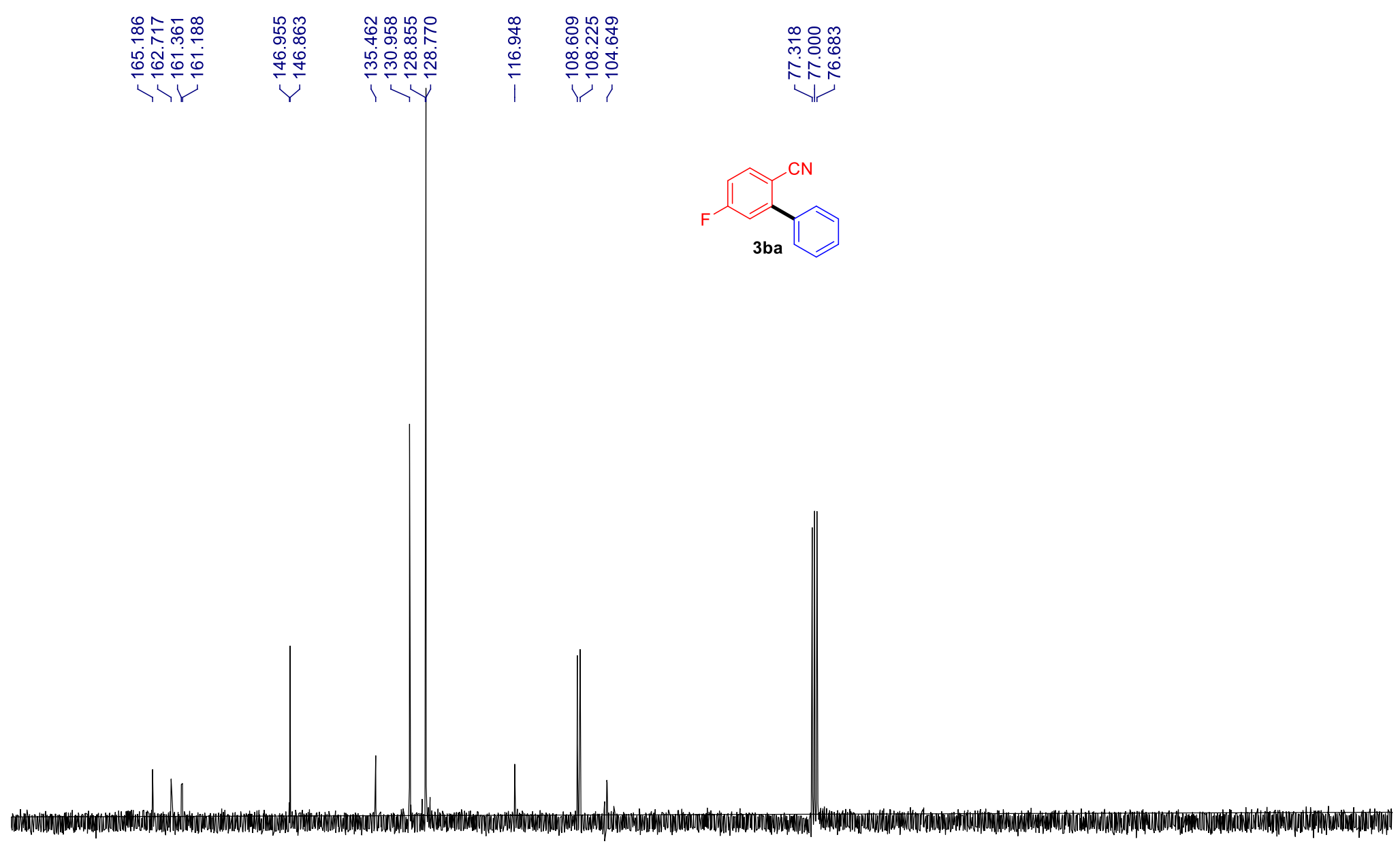

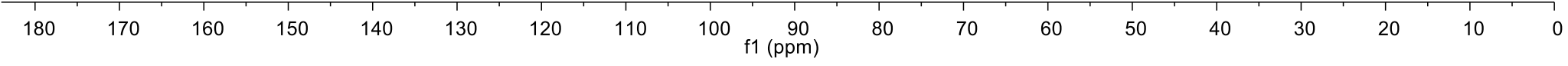



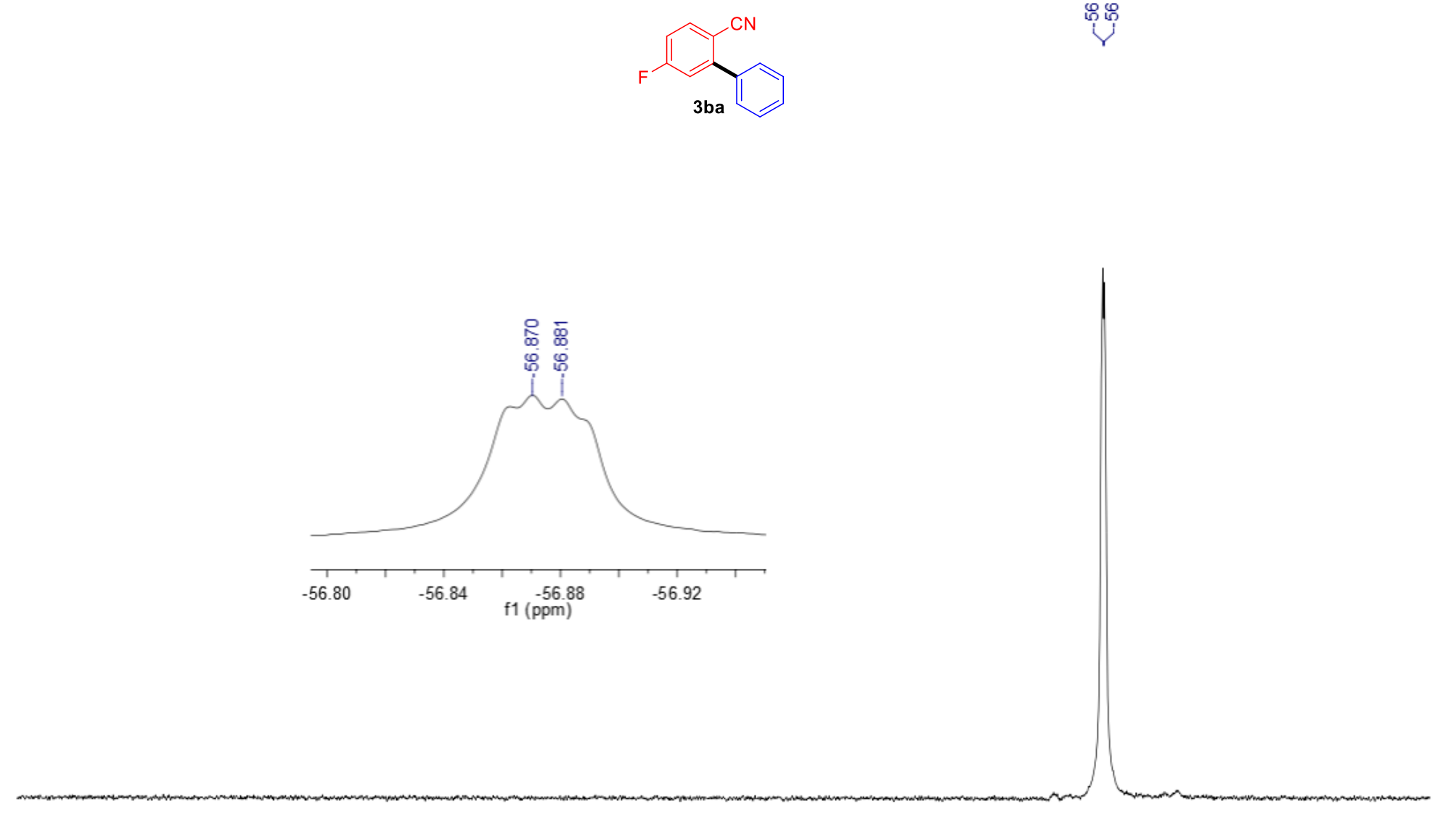

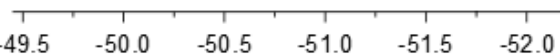




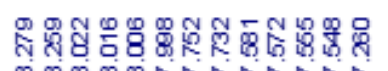

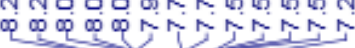

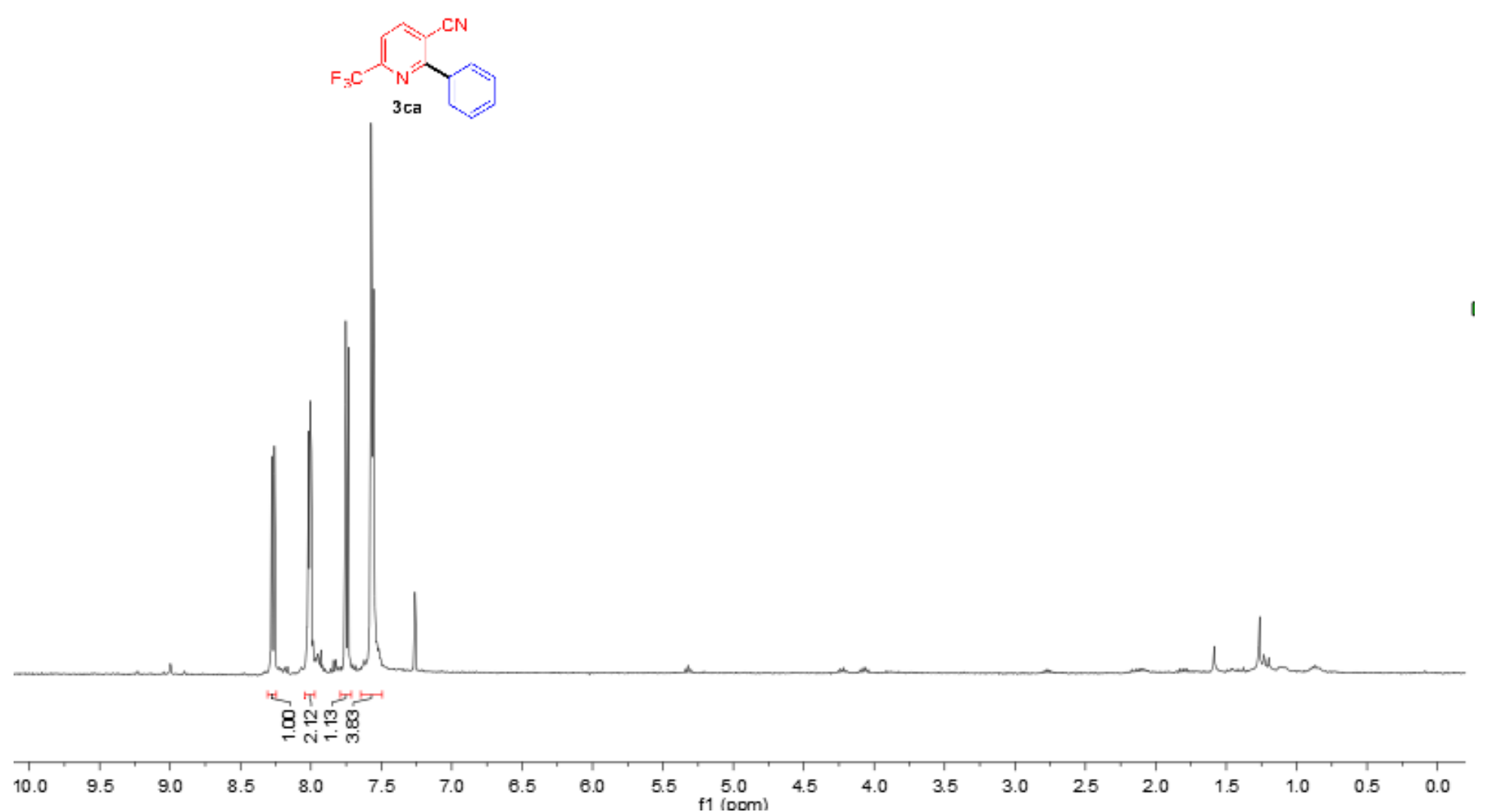




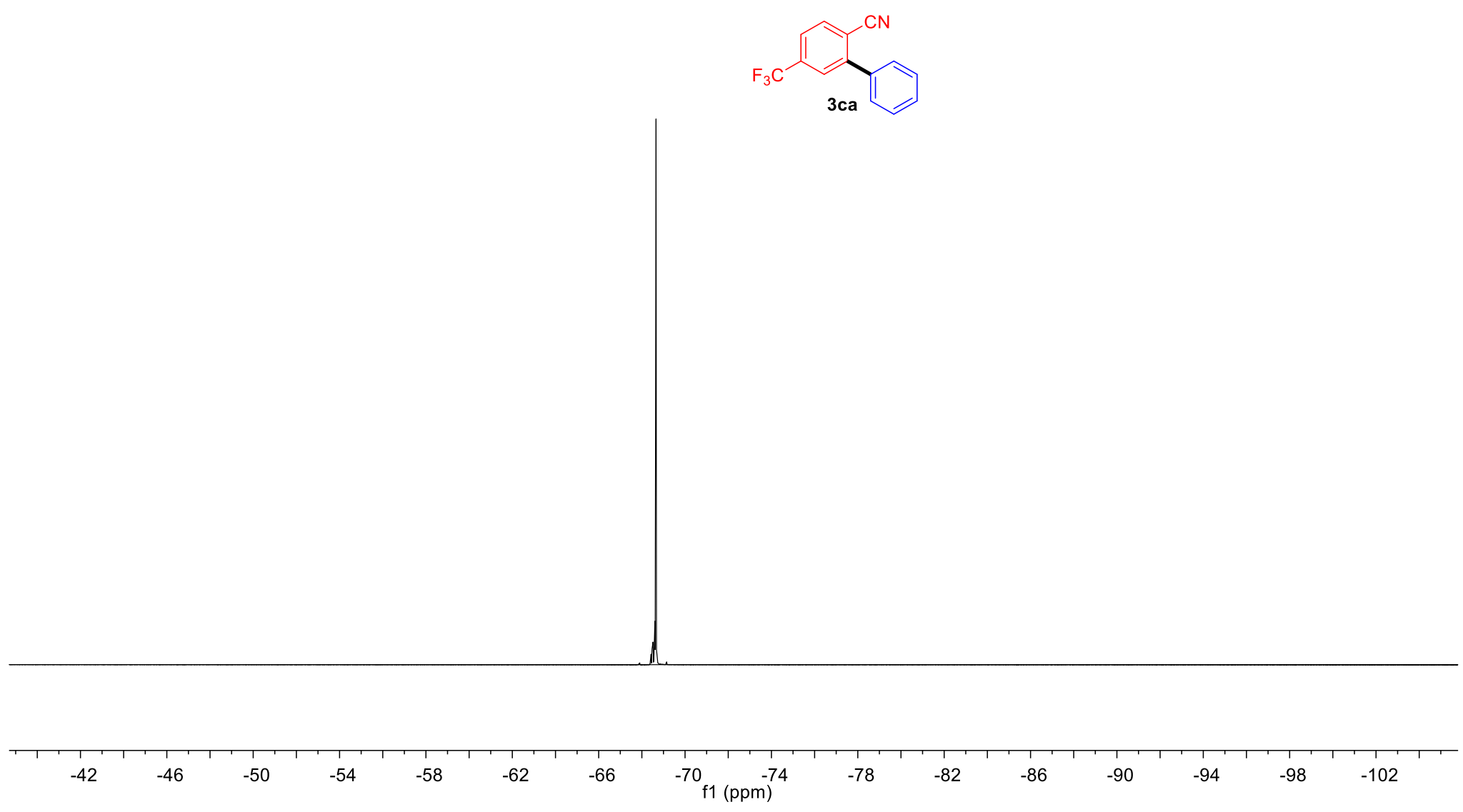


言的

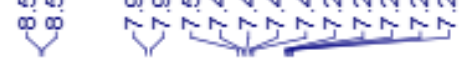
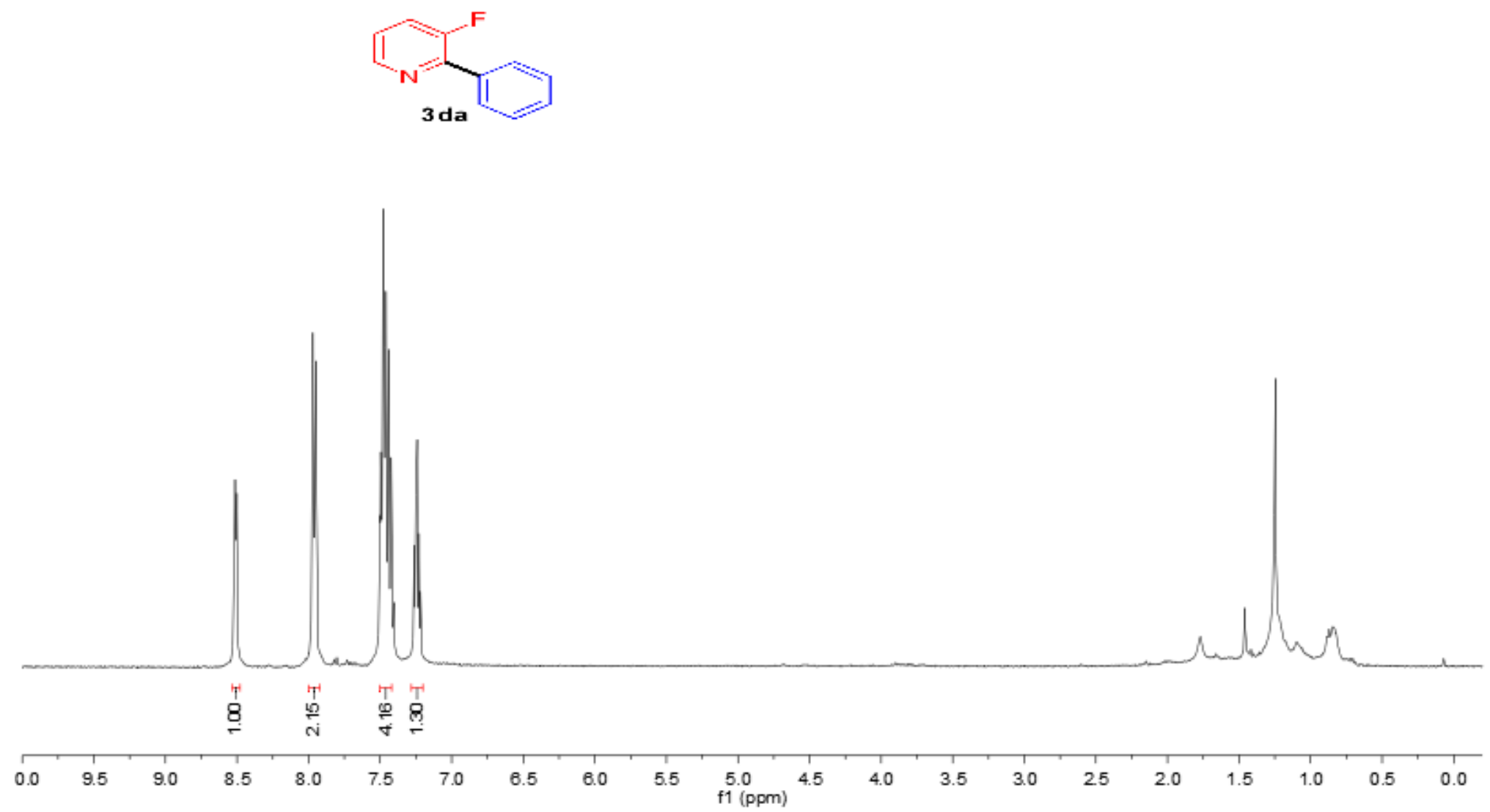

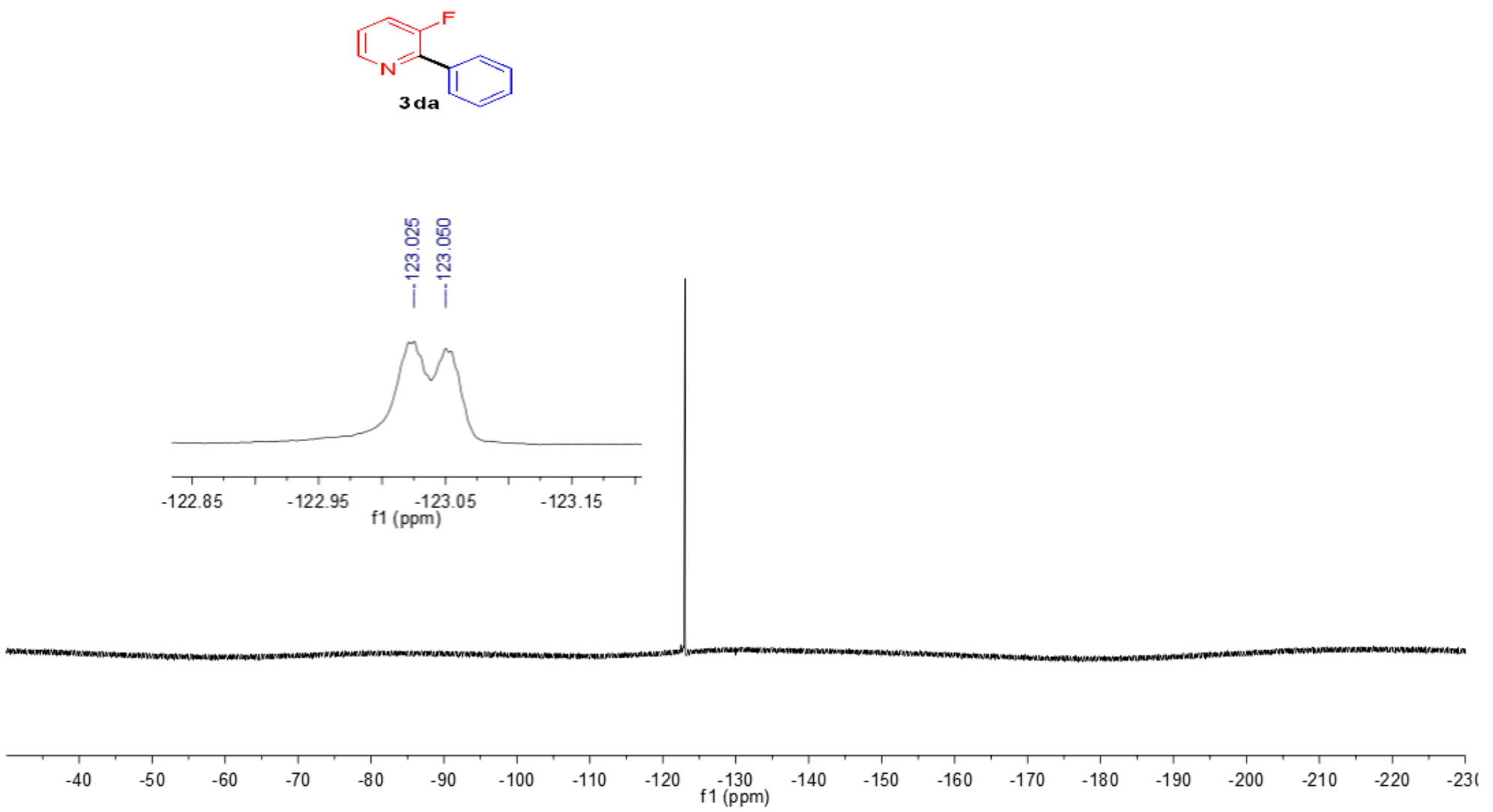


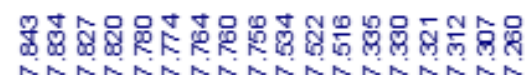

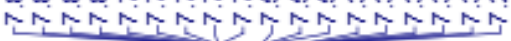

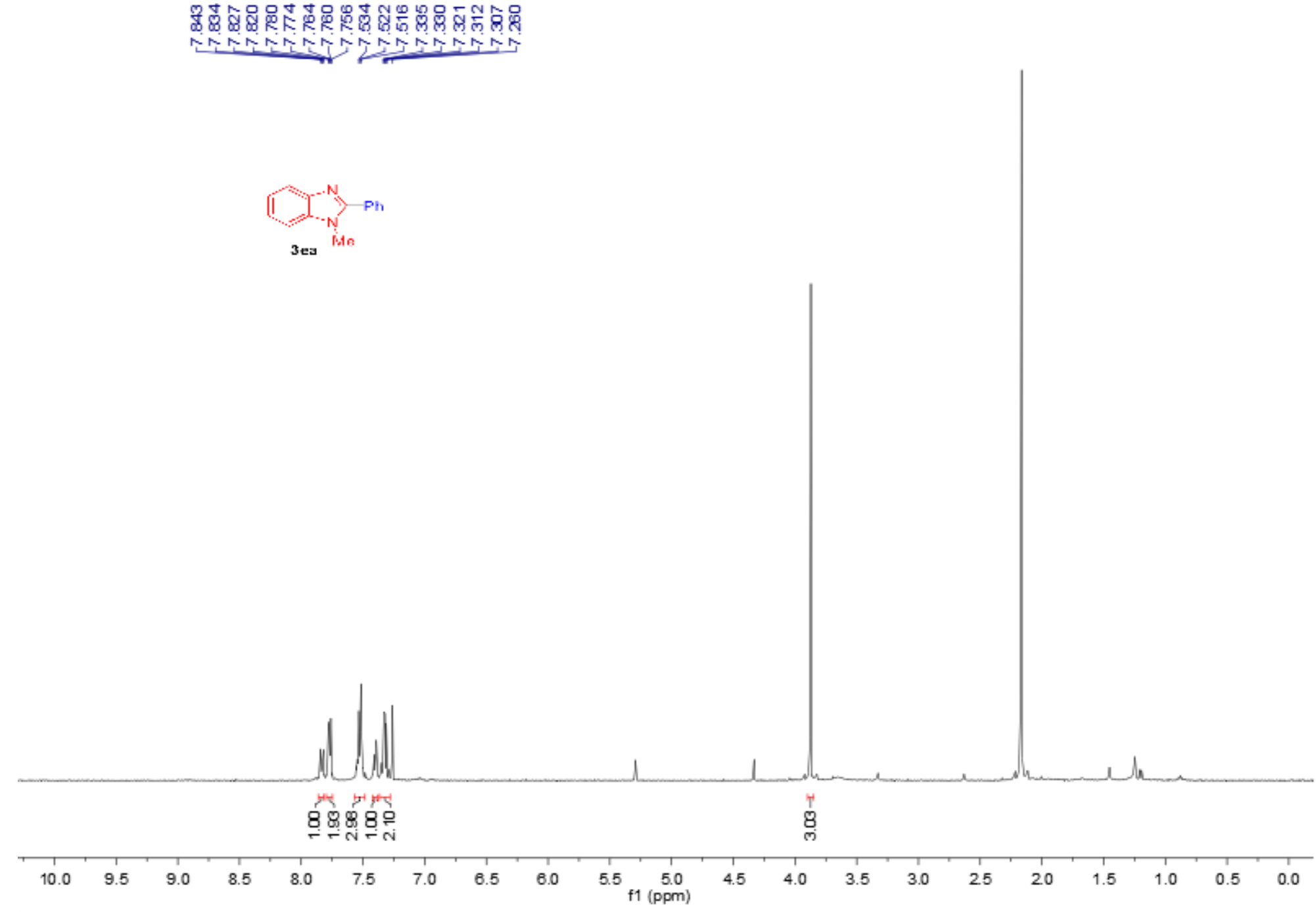




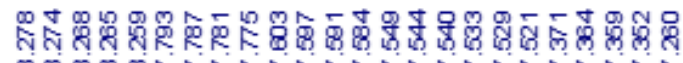

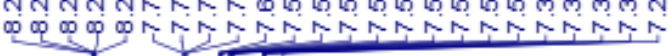

$\left[\sum_{3 \mathbf{f a}}^{-\mathrm{N}} \mathrm{Ph}\right.$

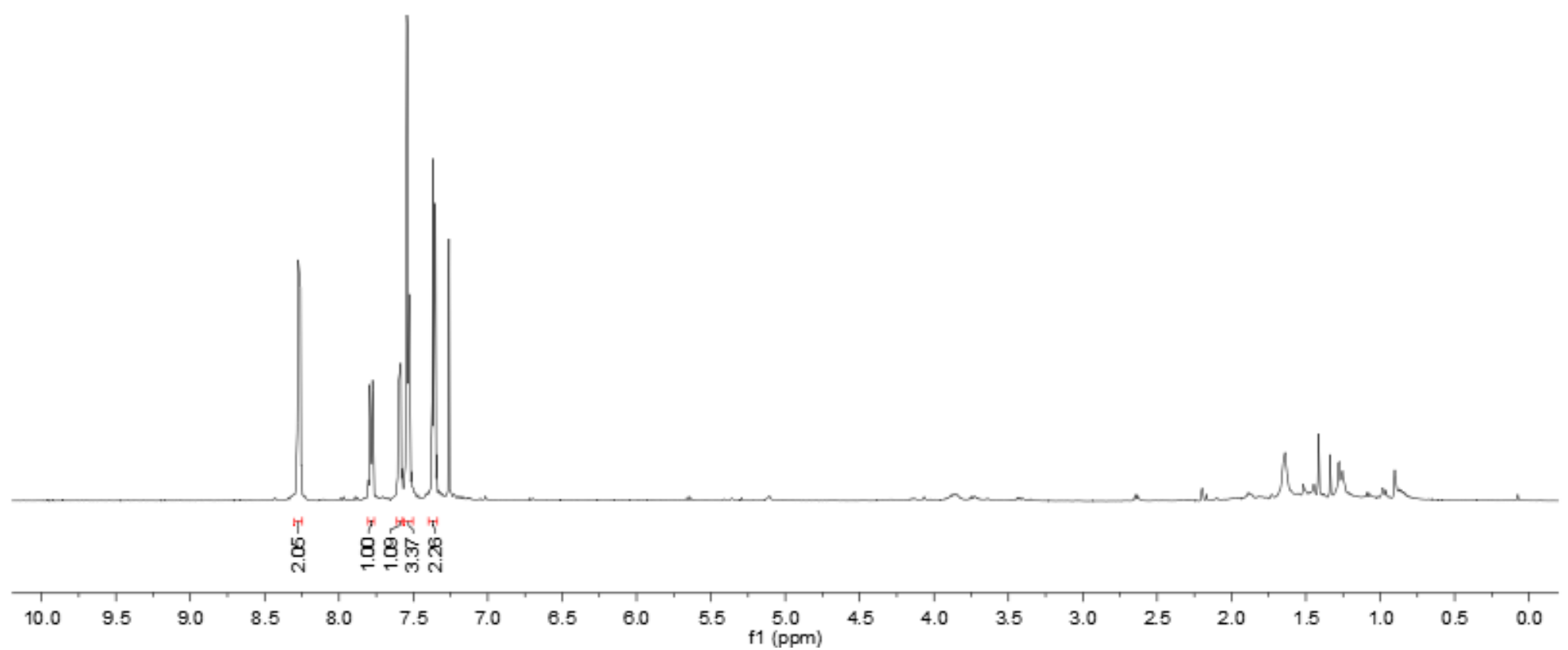




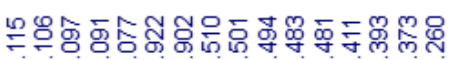

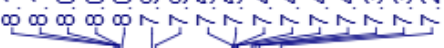

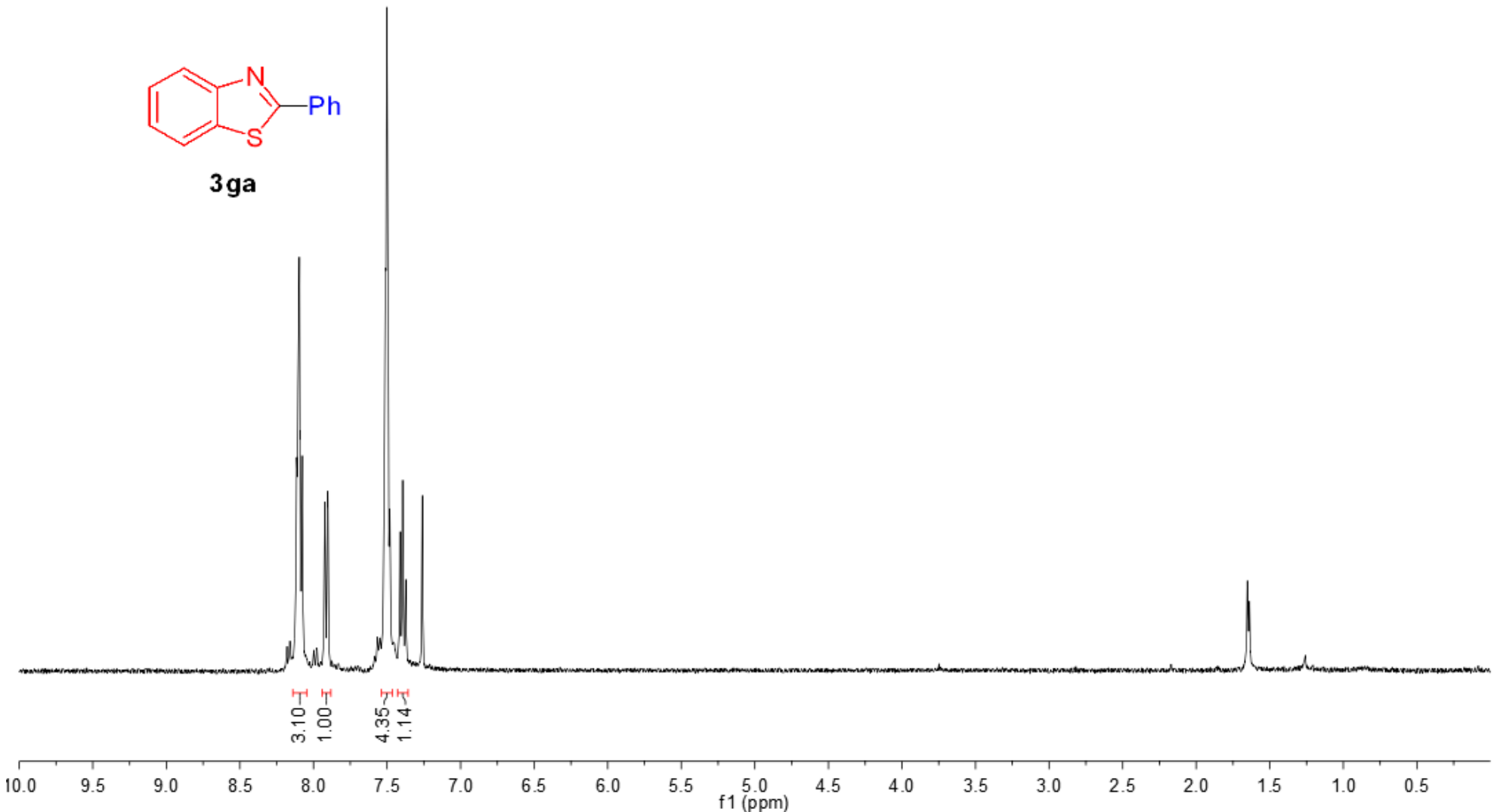




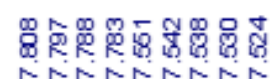

vondos

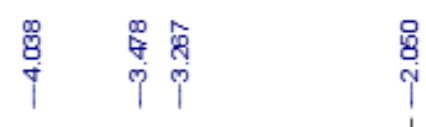

$$
\text { 3ha }
$$

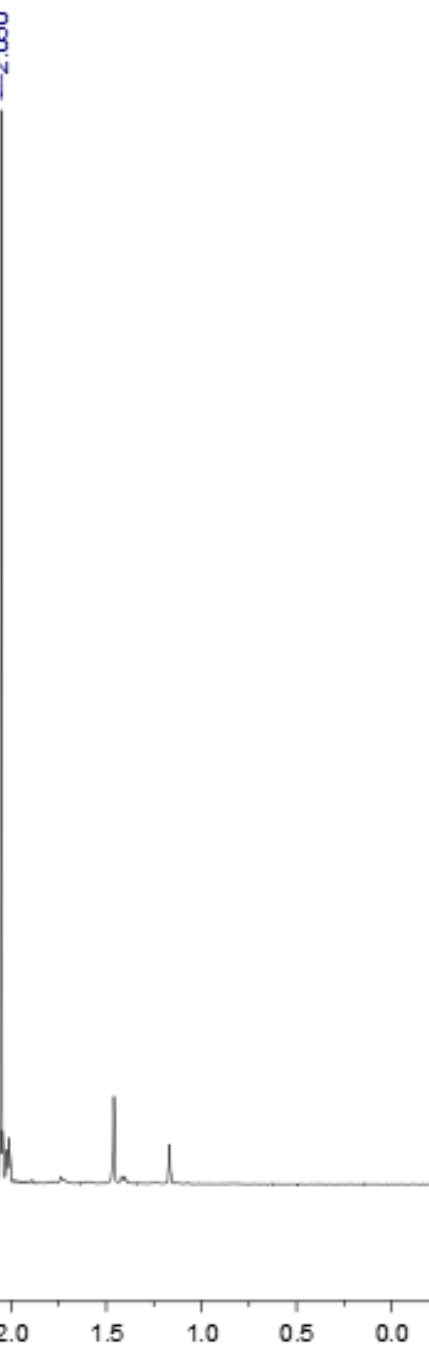




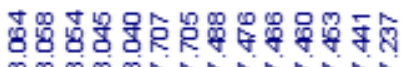

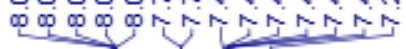

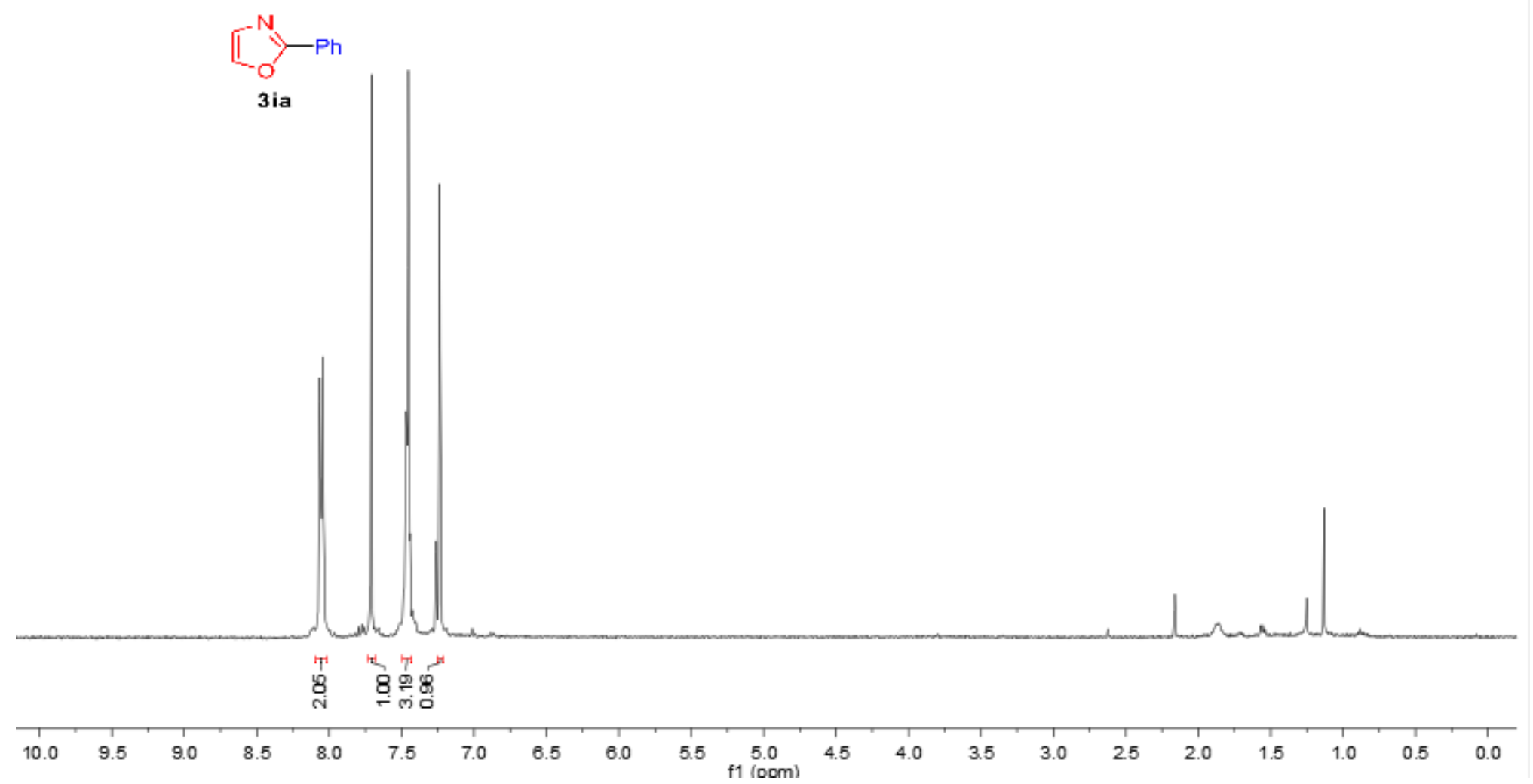




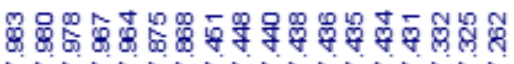

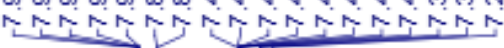
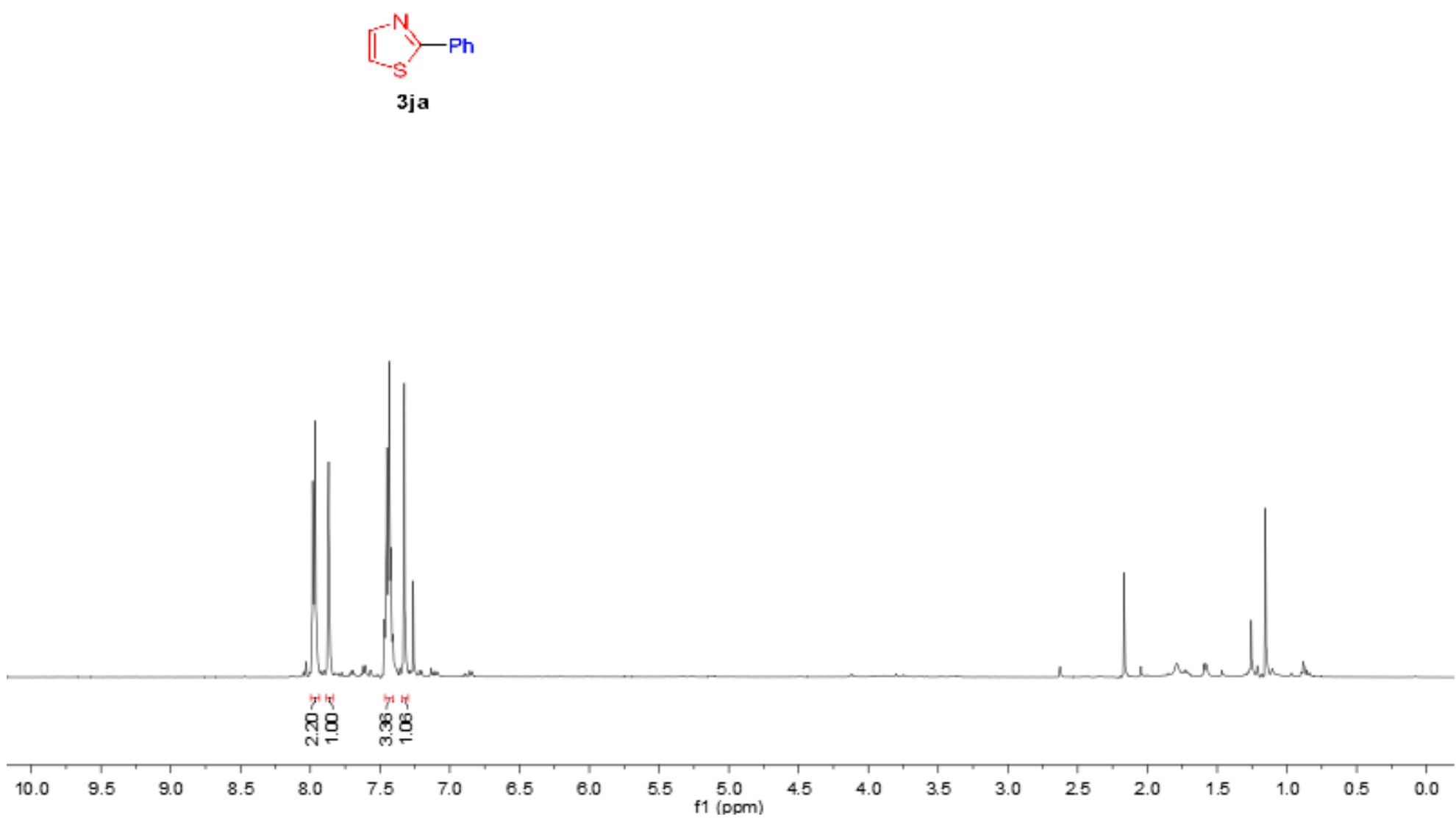


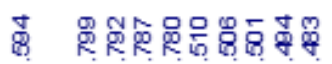

क varivar

$$
\overbrace{\mathbf{3 k a}}^{\mathrm{N}} \mathrm{Ph}
$$

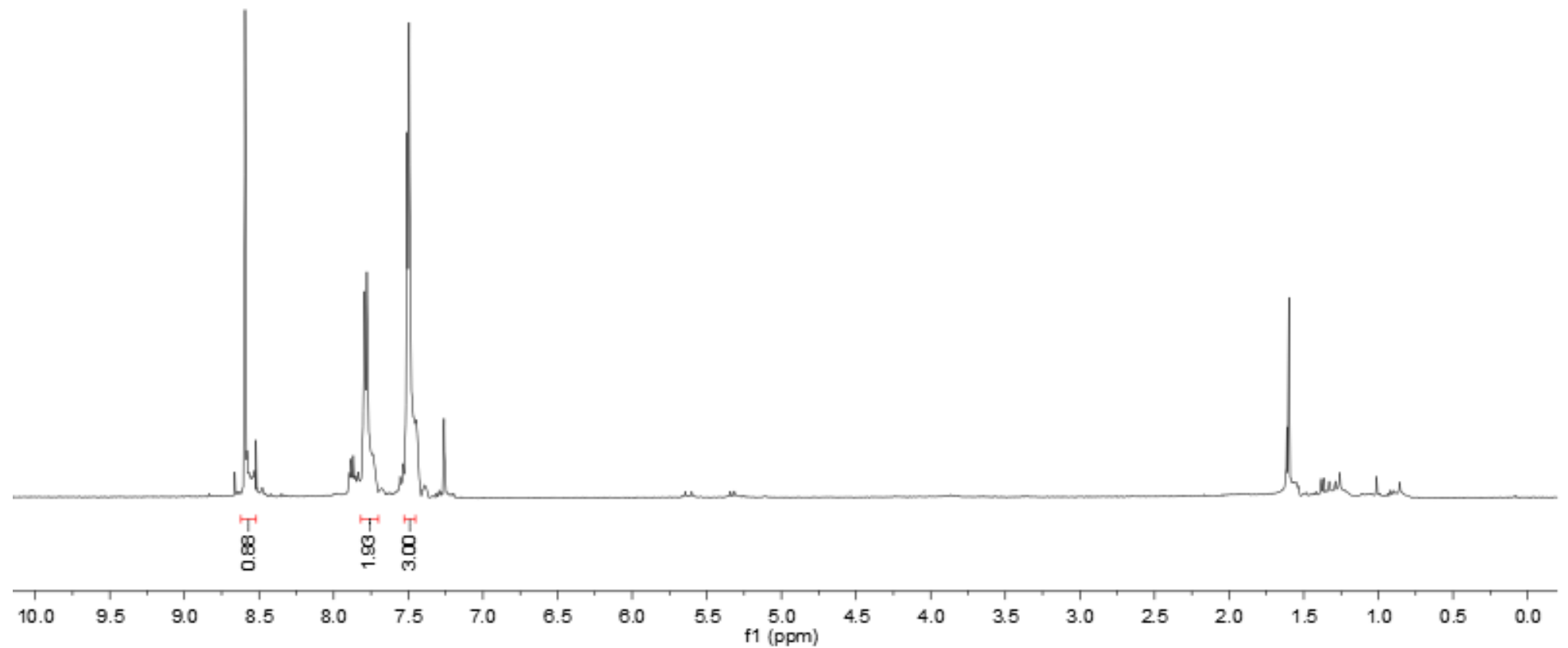




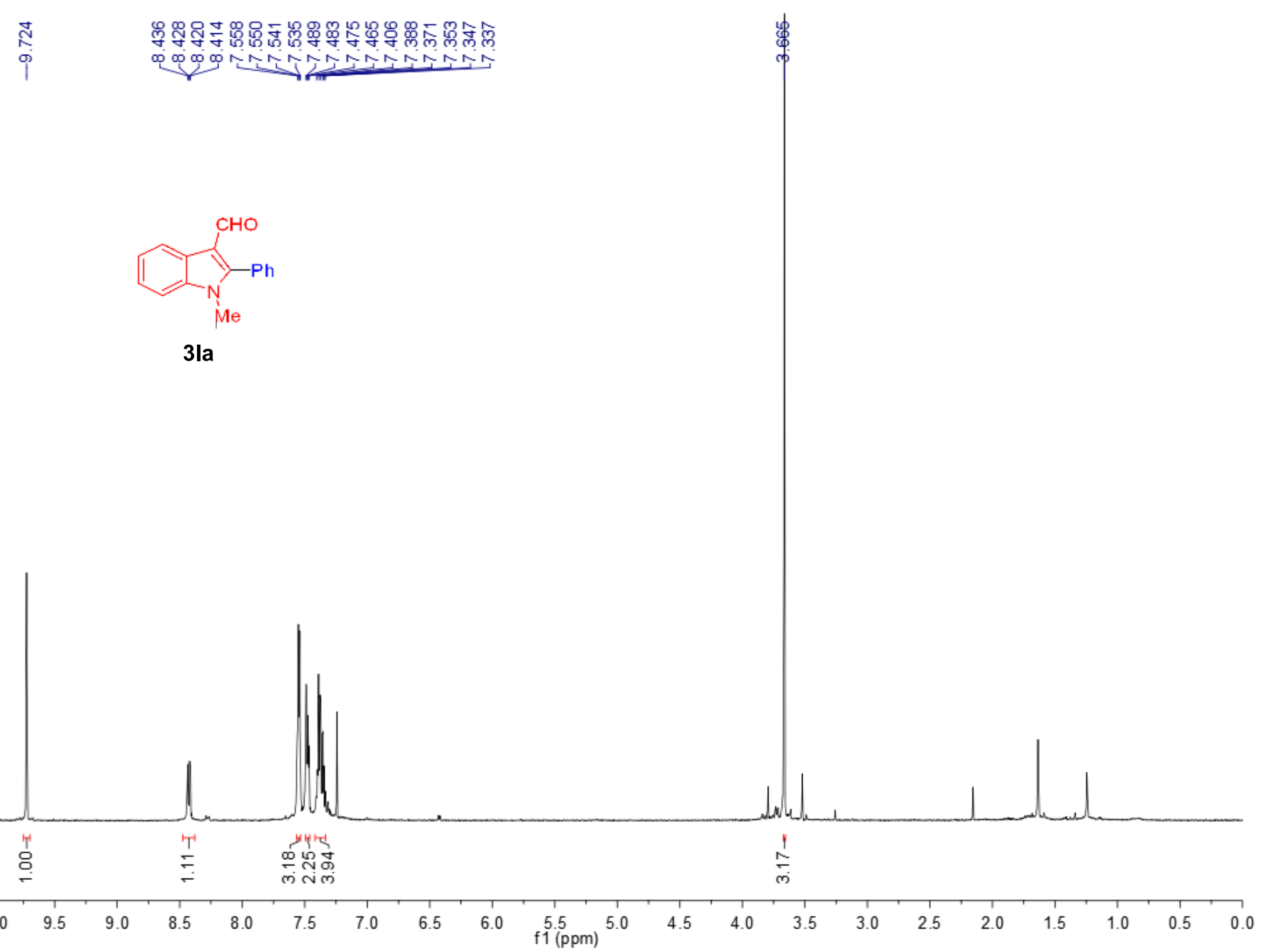




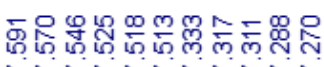

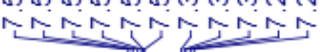

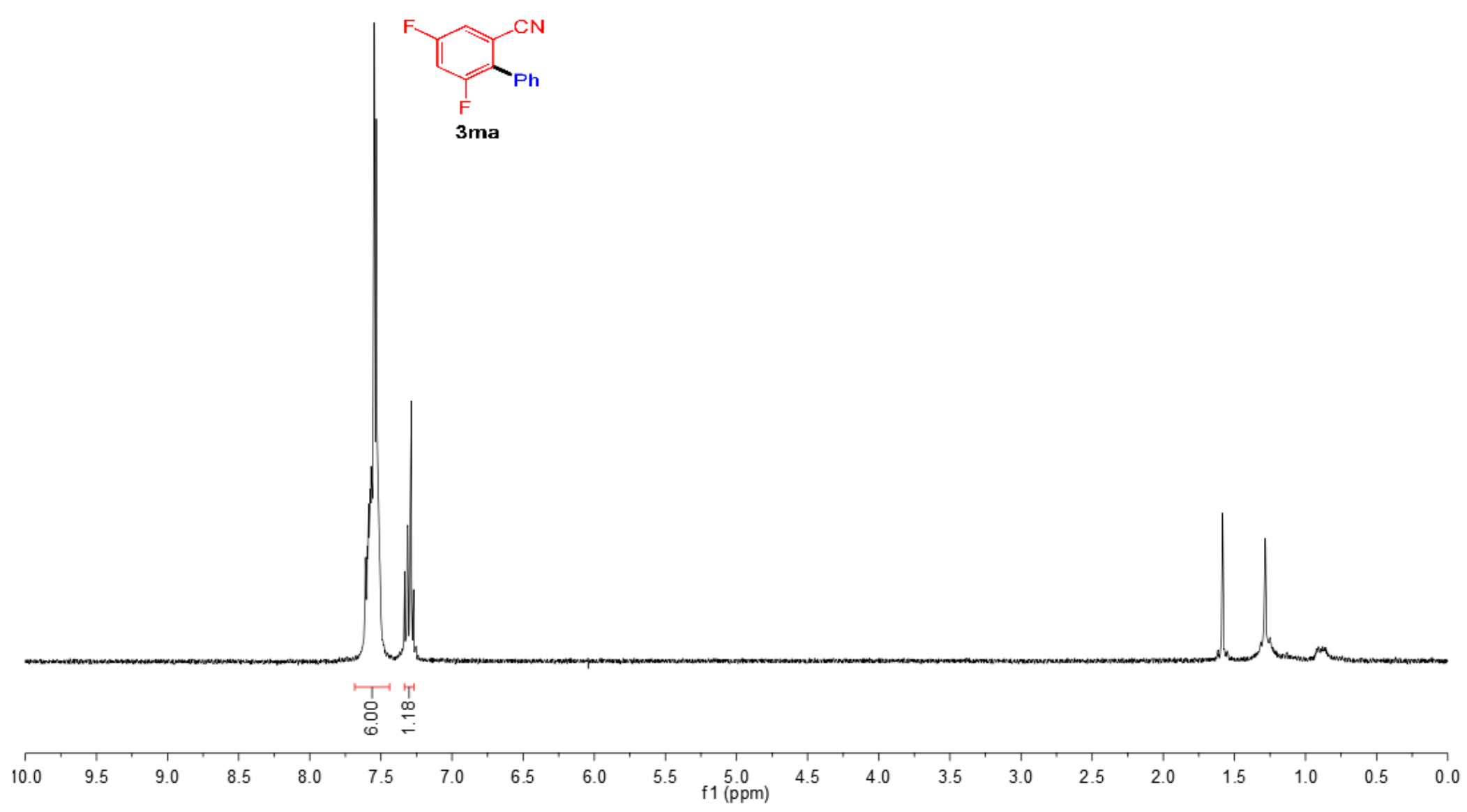




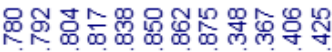

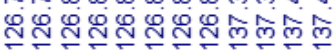

i i i - $\div$
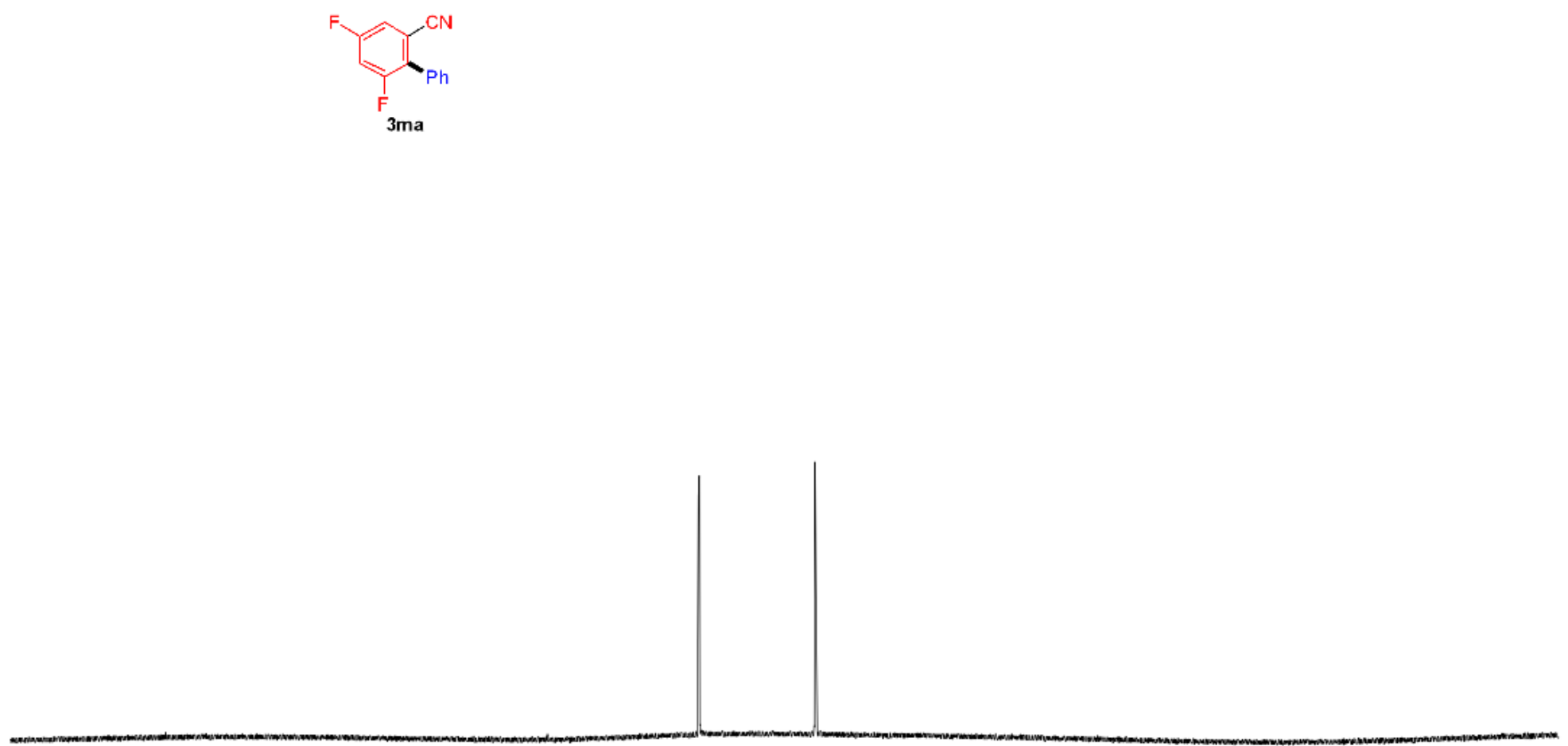


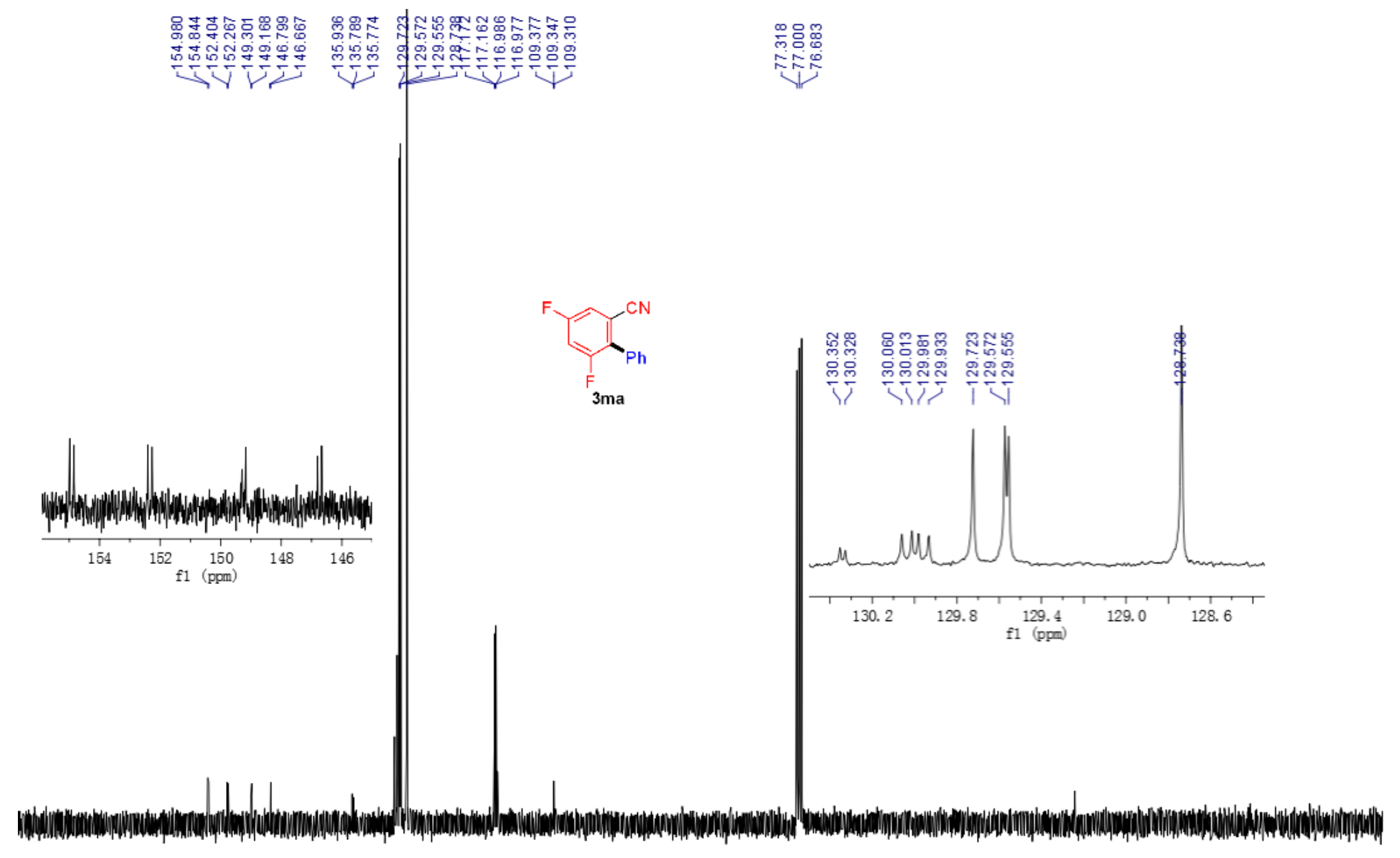




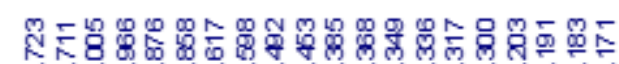

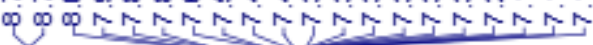
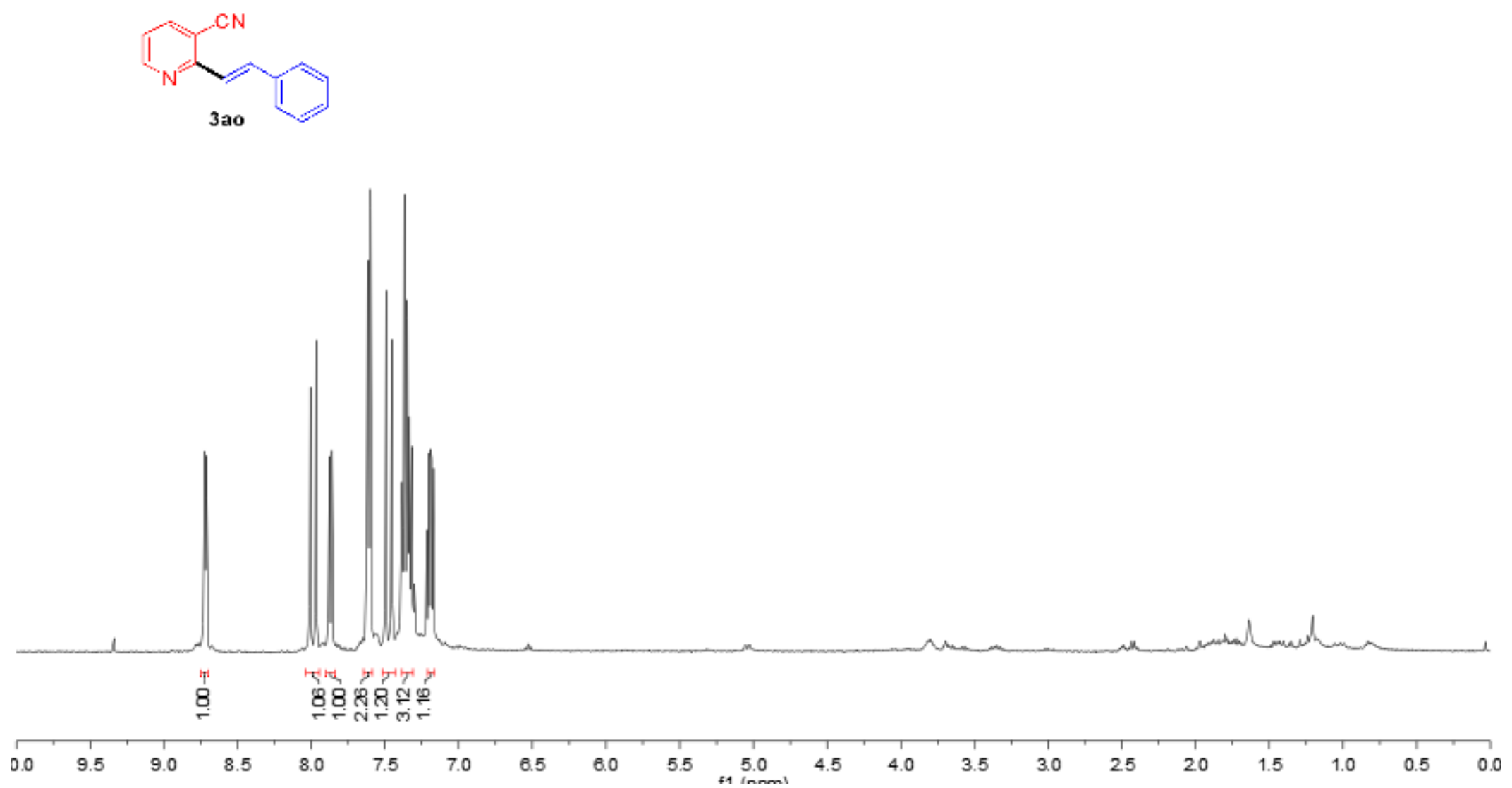


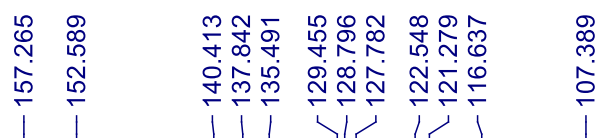

ํํㅇㅇㅛ

रํํ

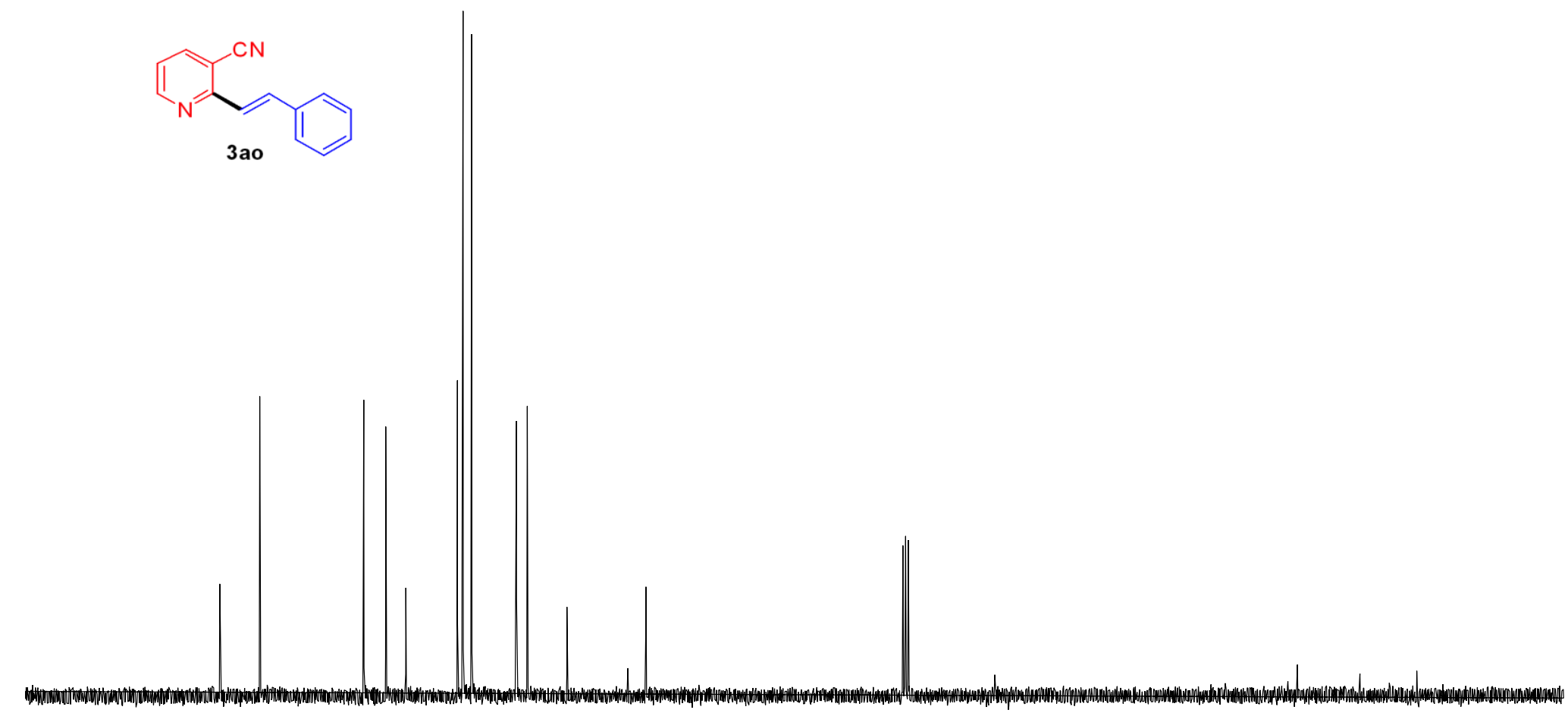

How 


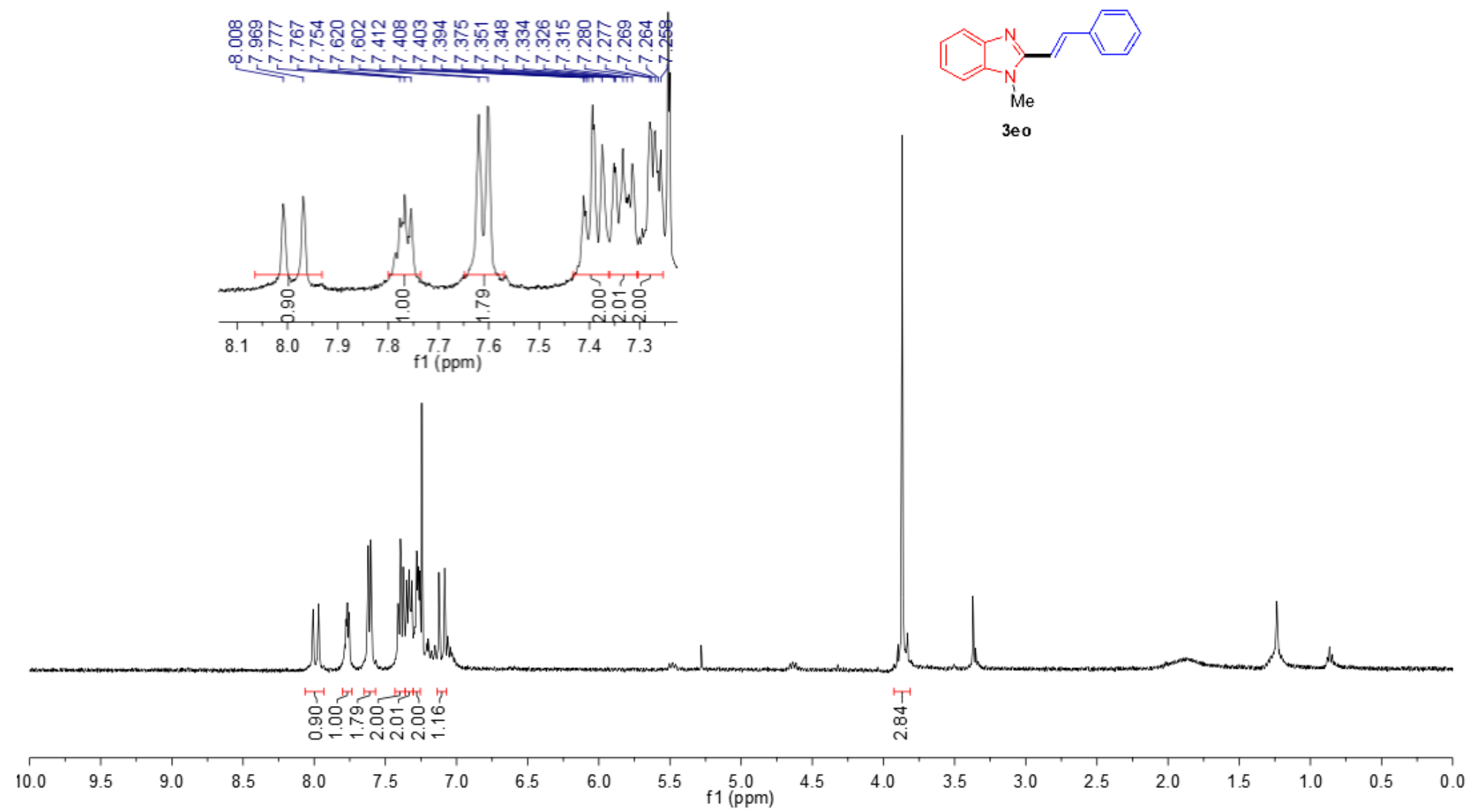




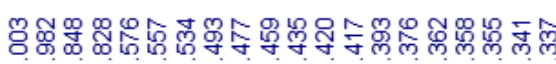

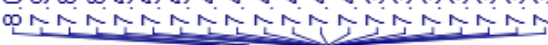
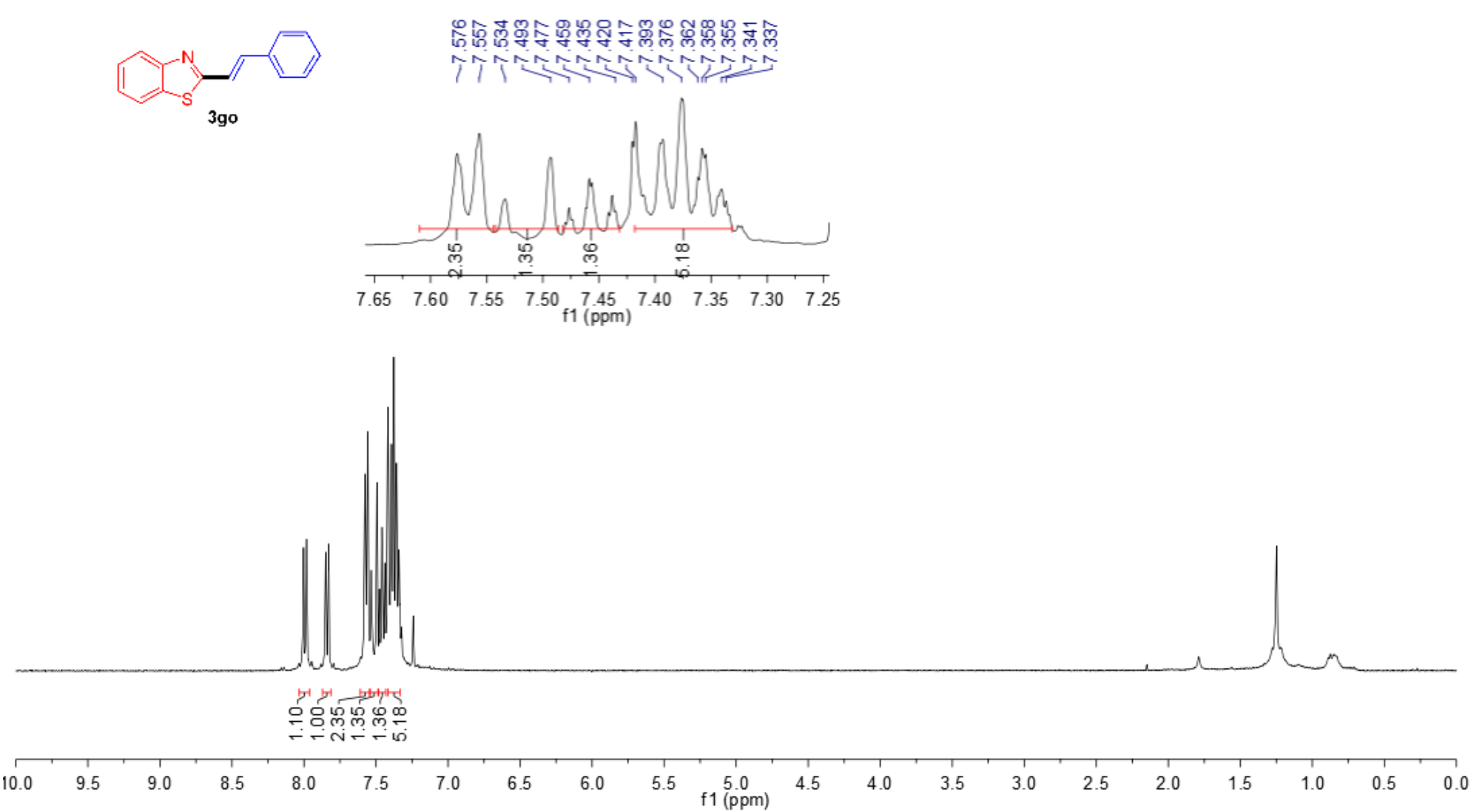


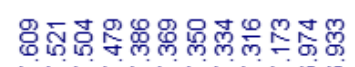

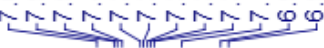
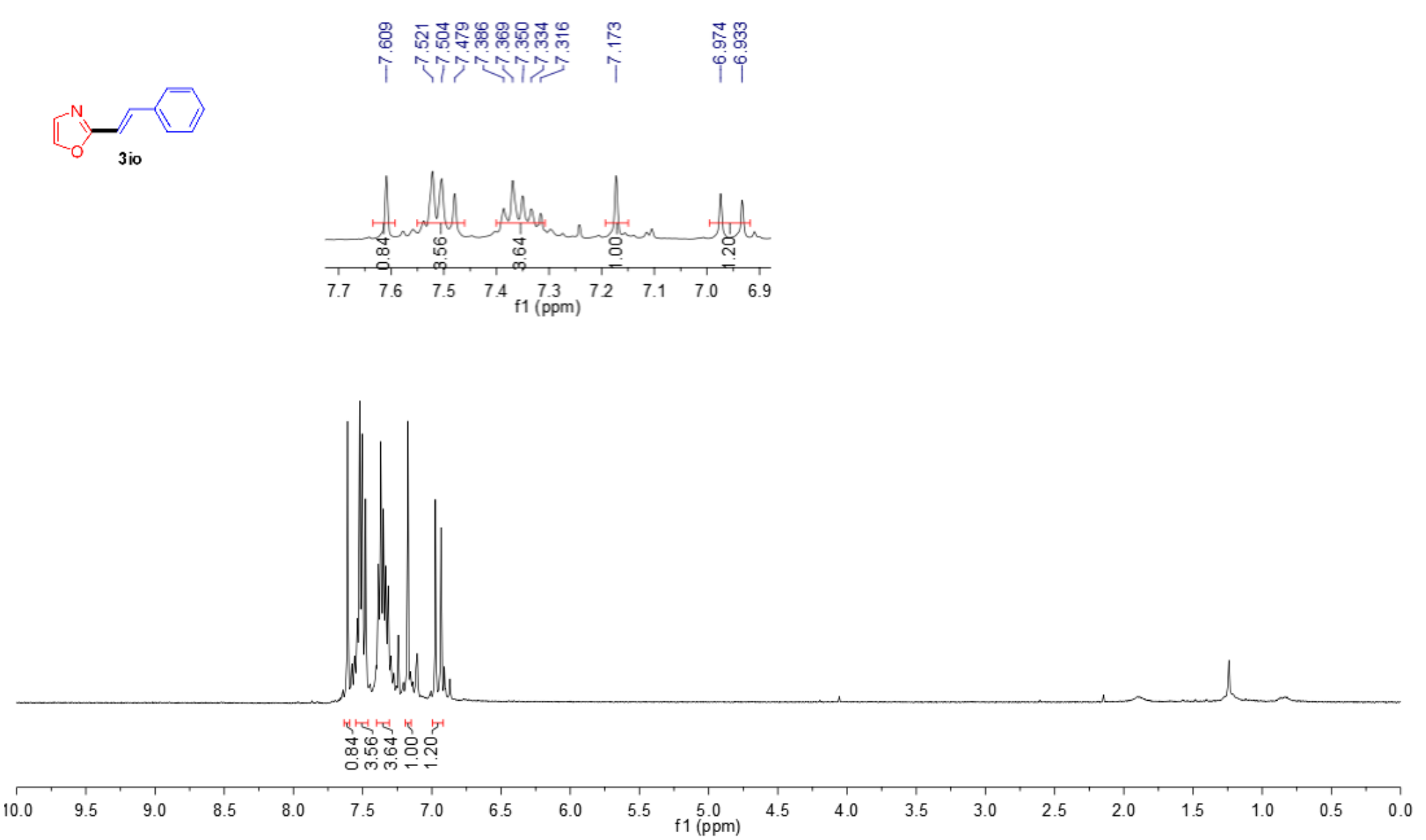


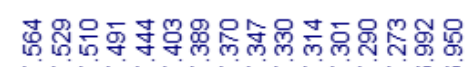

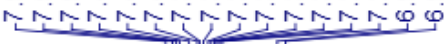

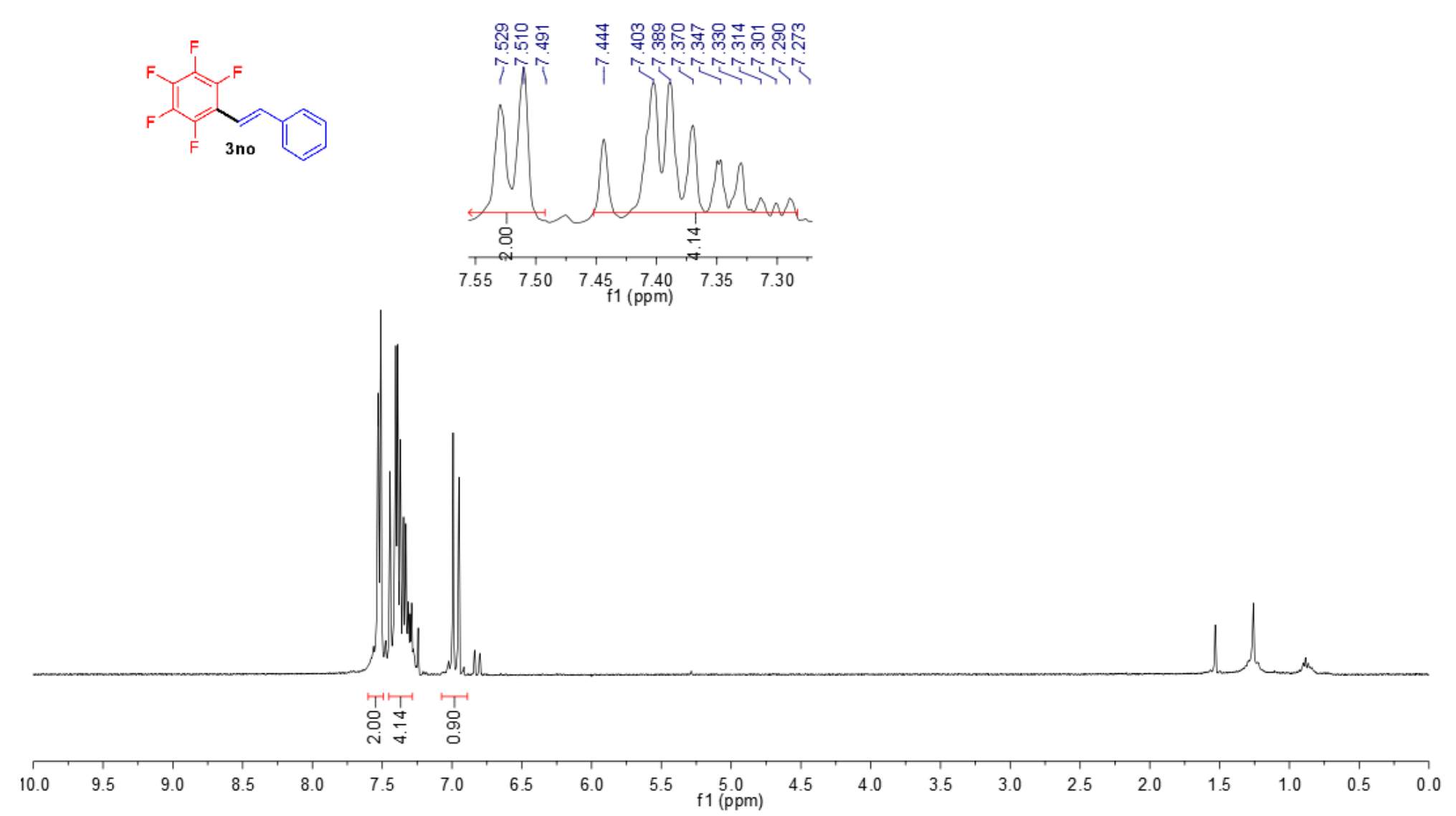




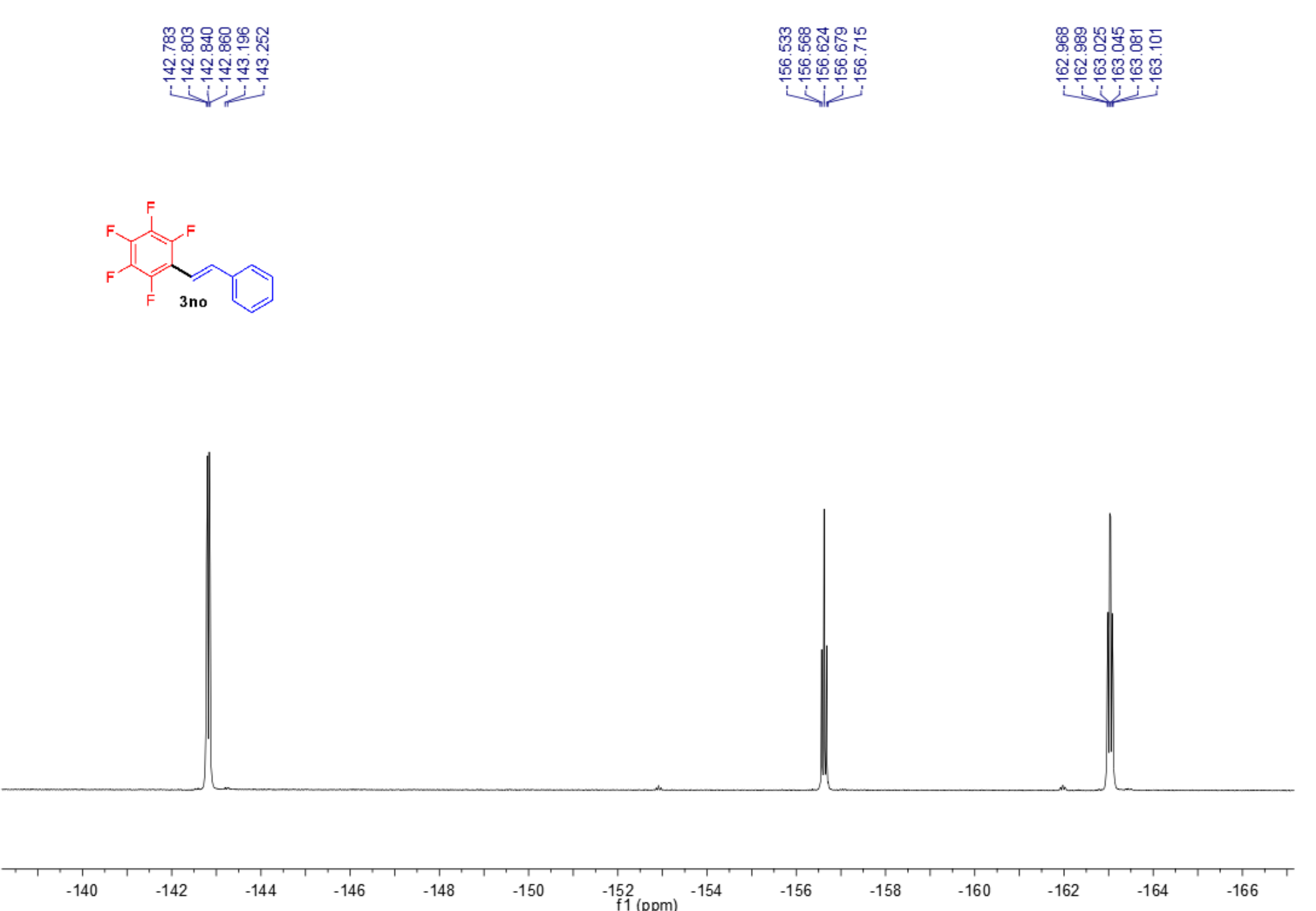




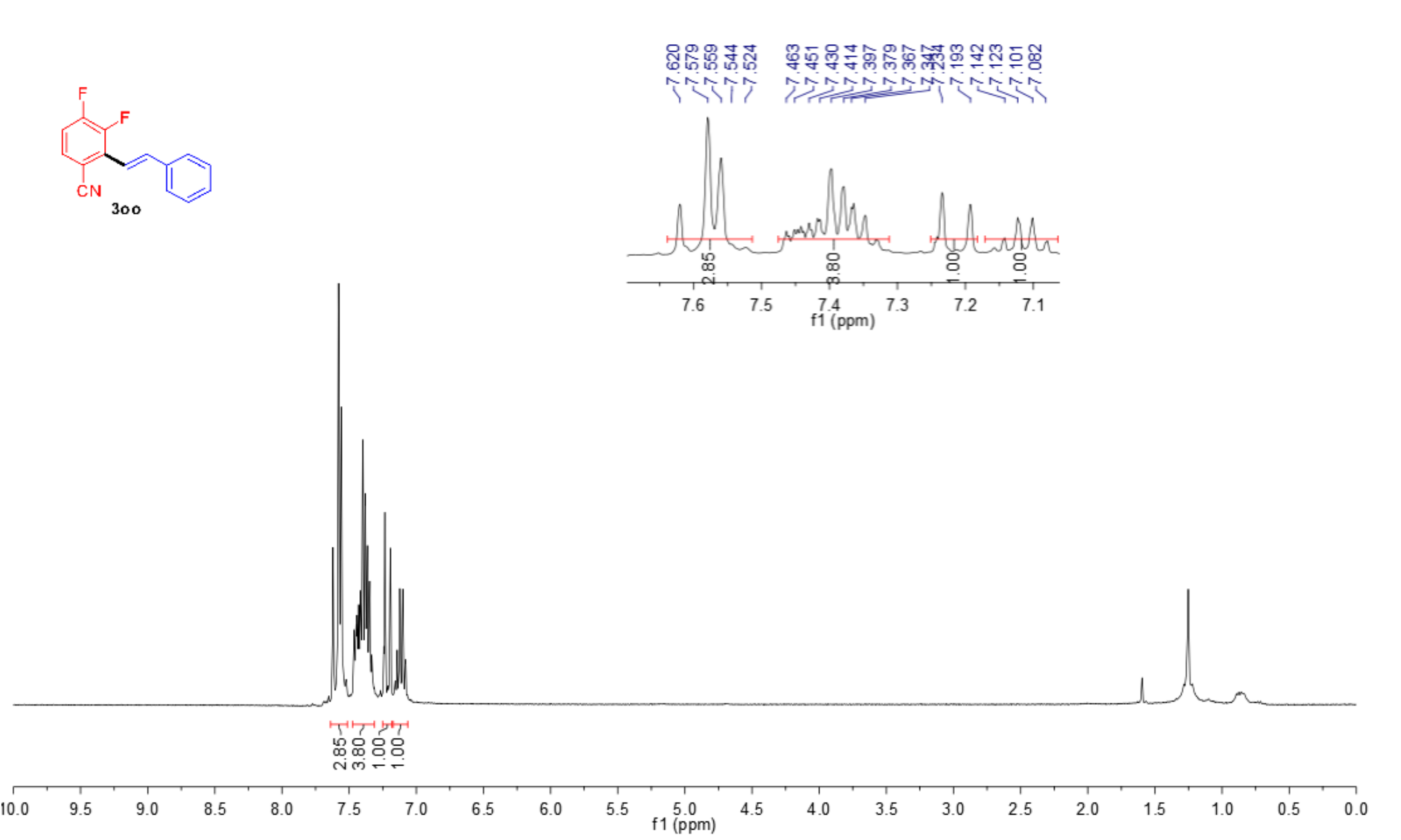




$$
\pm
$$



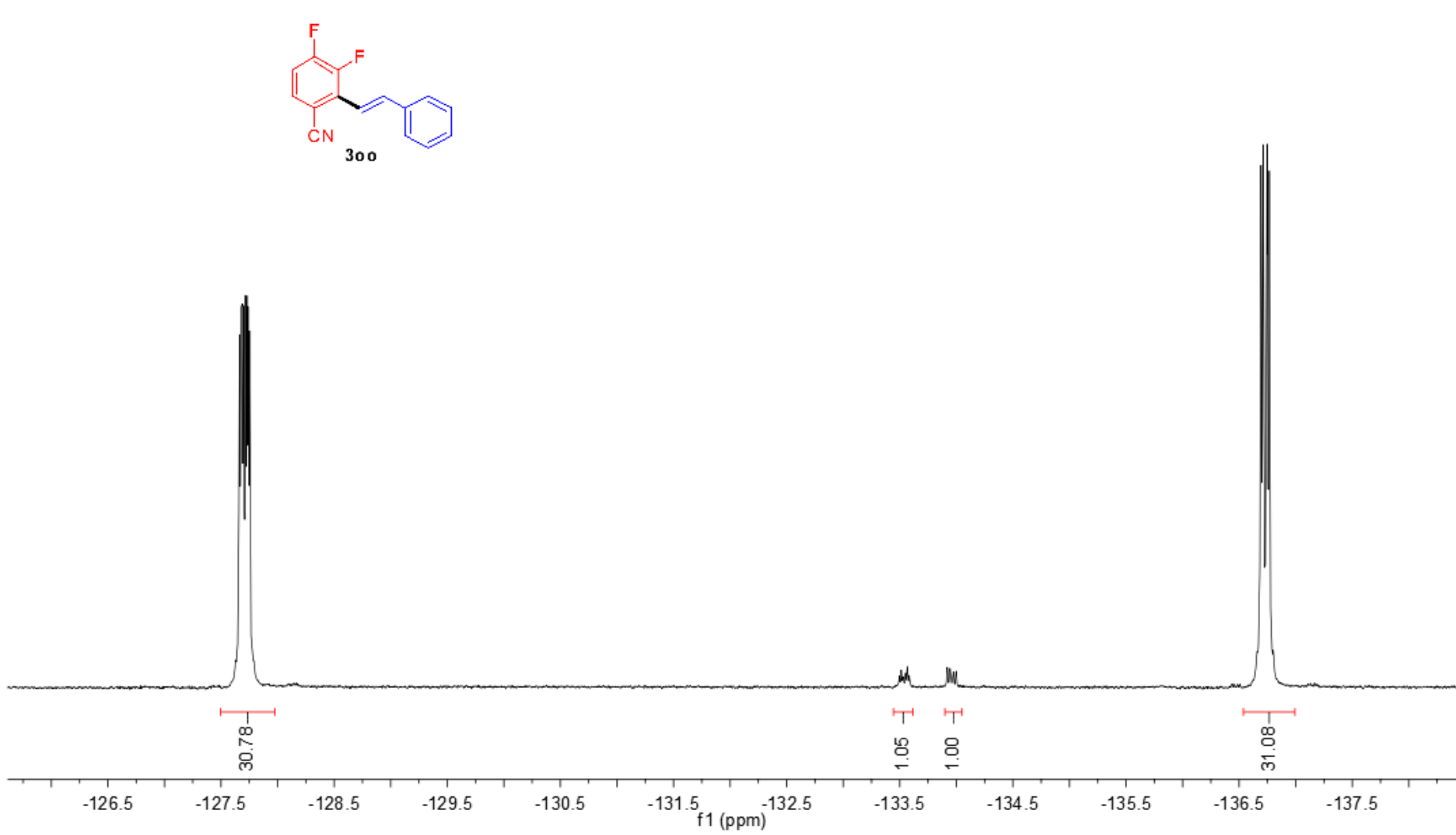

Note: The sample contains $\sim 3 \%$ impurity (possibly the isomer resulting from the regioselectivity of zincation/vinylation). 


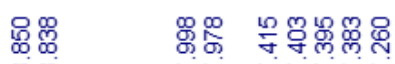

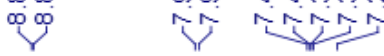

$$
\underbrace{C N}_{\text {3ap }}
$$

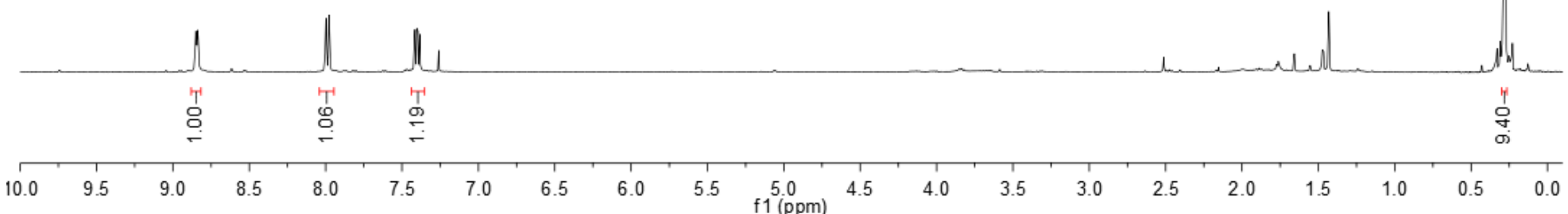




$$
\underset{\substack{N \\ \text { iep }}}{-N}=\text { TMS }
$$

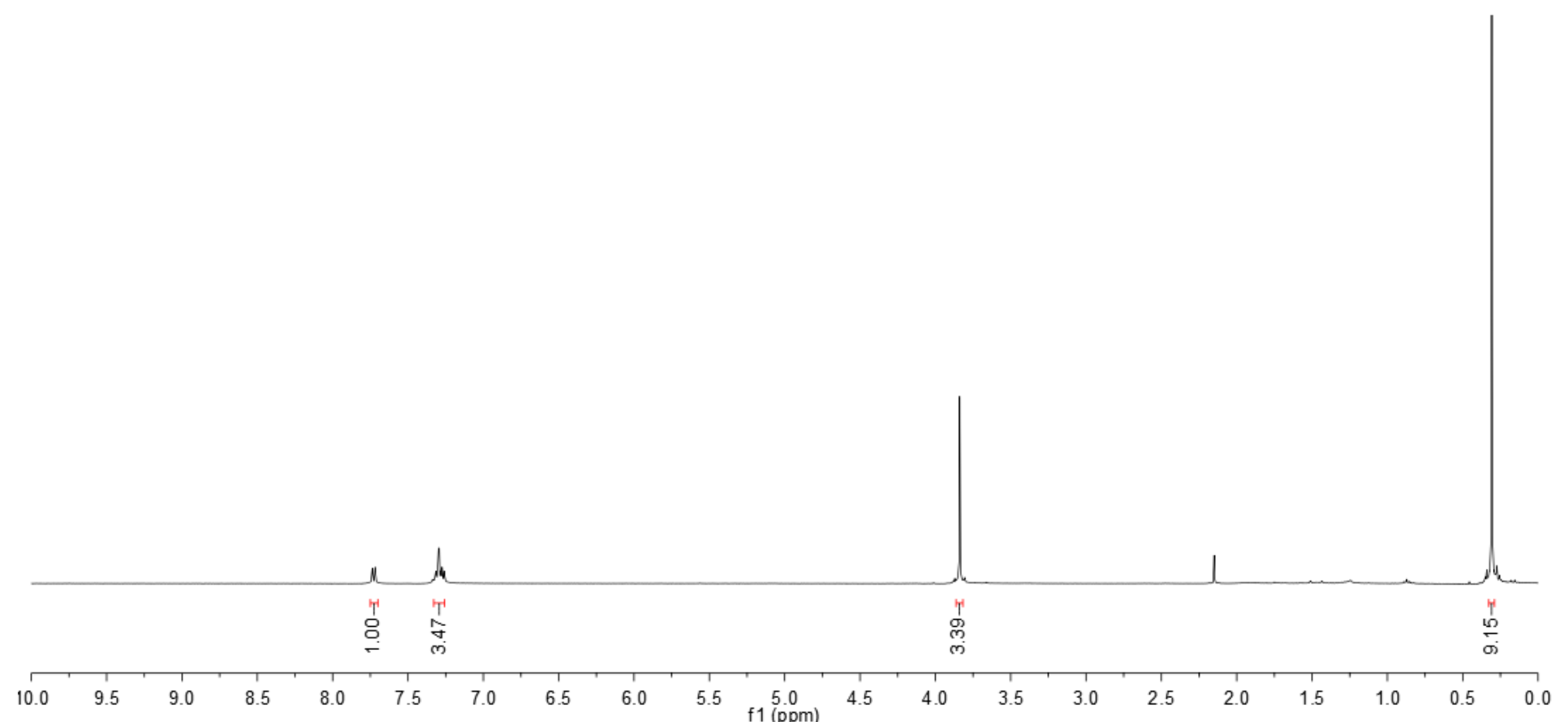




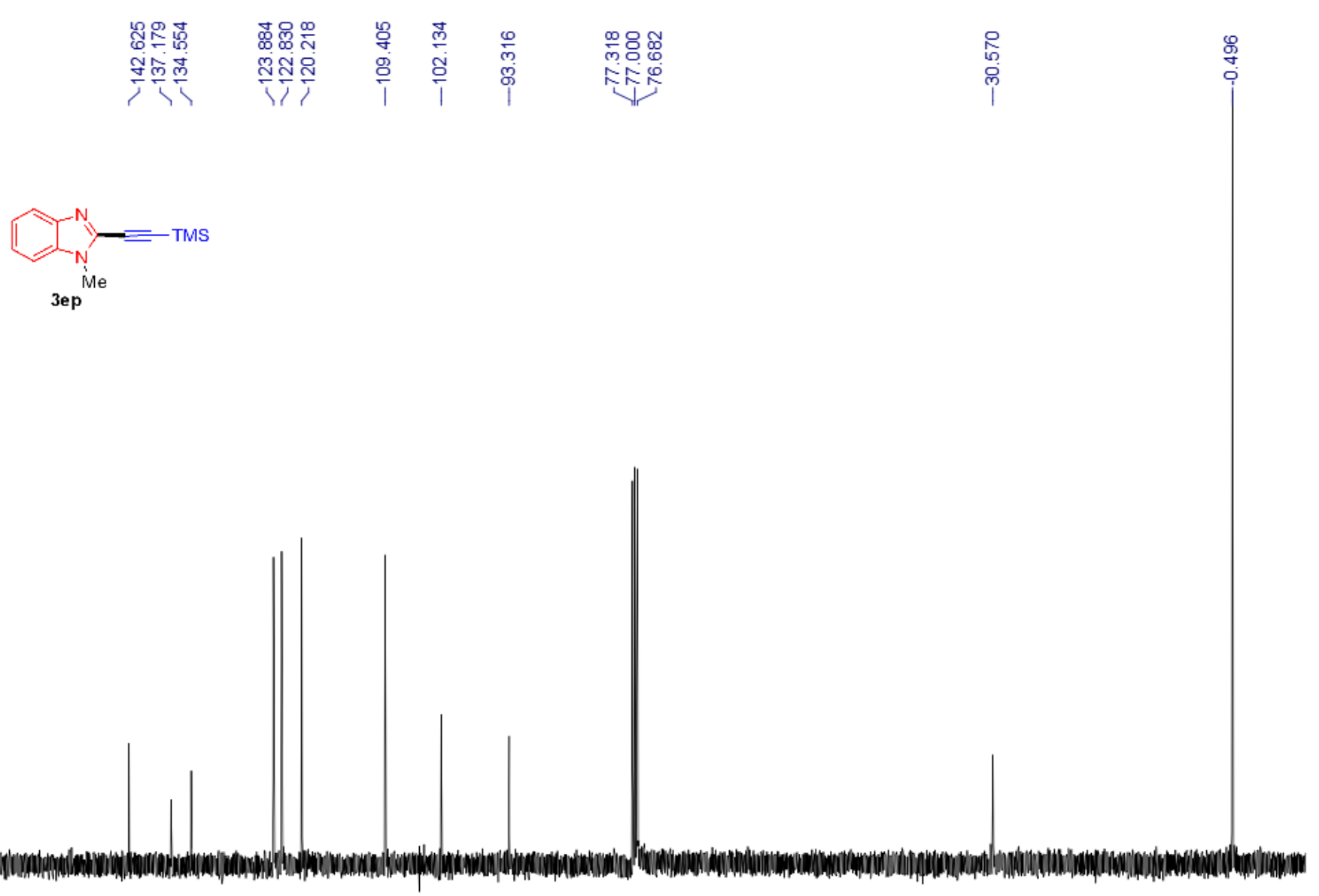

180

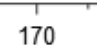

150

130

110

100

${ }_{\mathrm{f} 1}^{90}(\mathrm{ppm})$

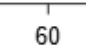

40 


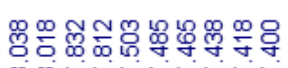

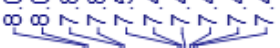

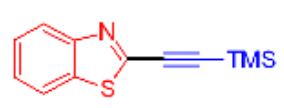

$3 g p$
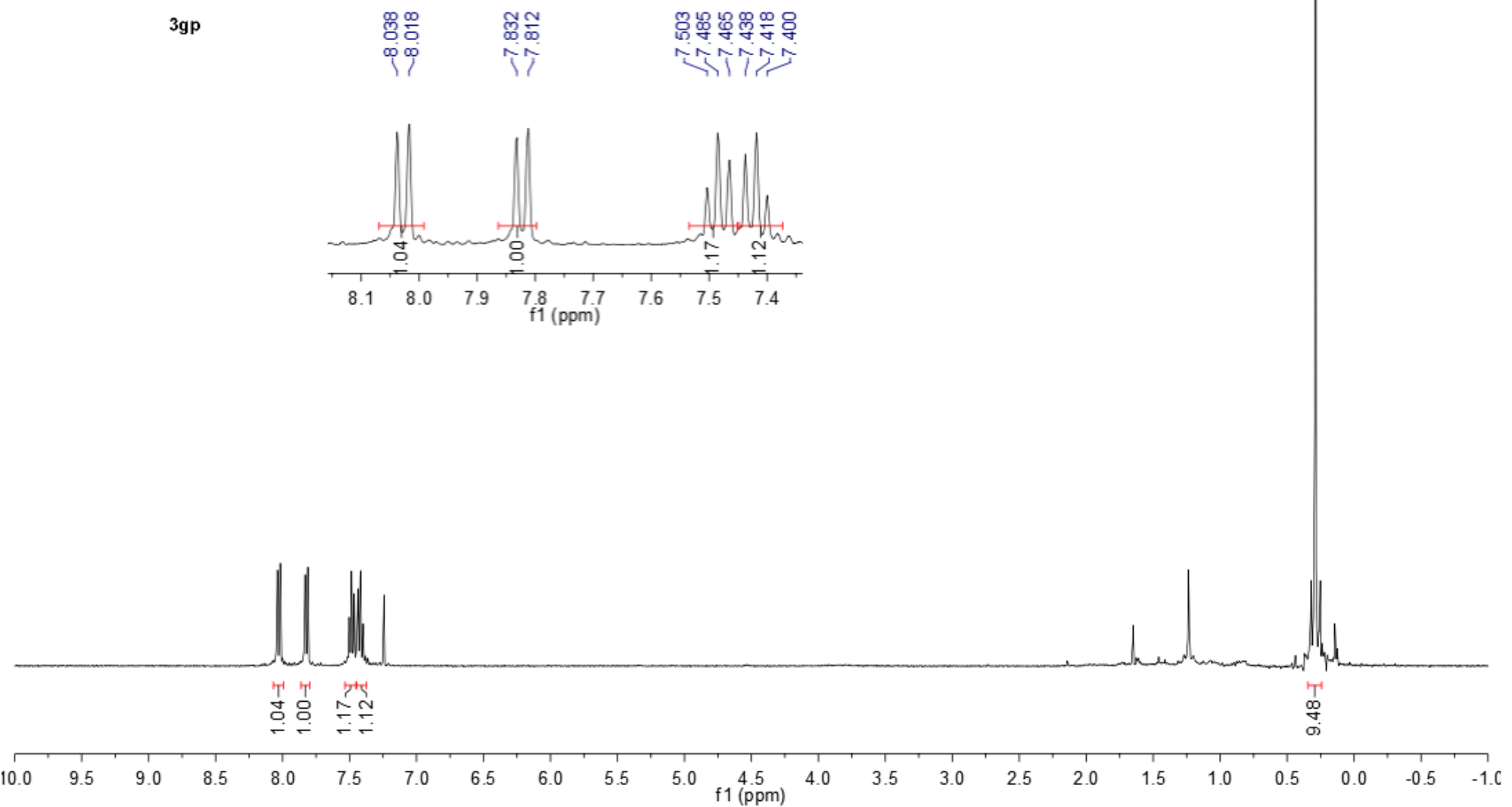


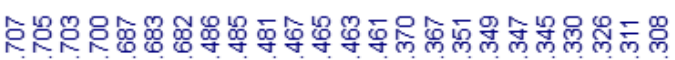

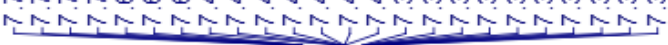
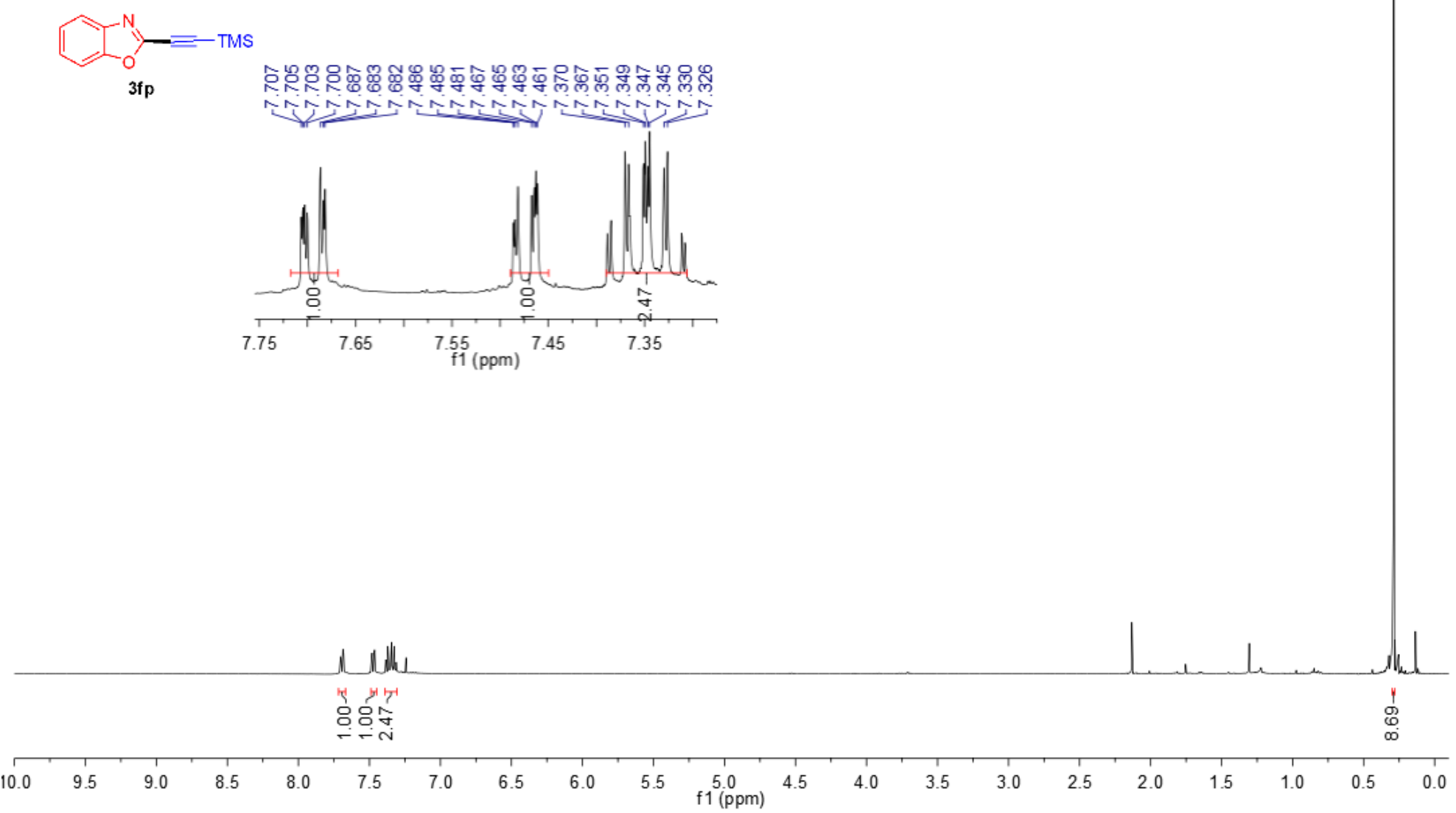


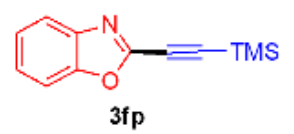

$\mid$ 


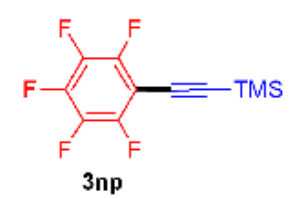




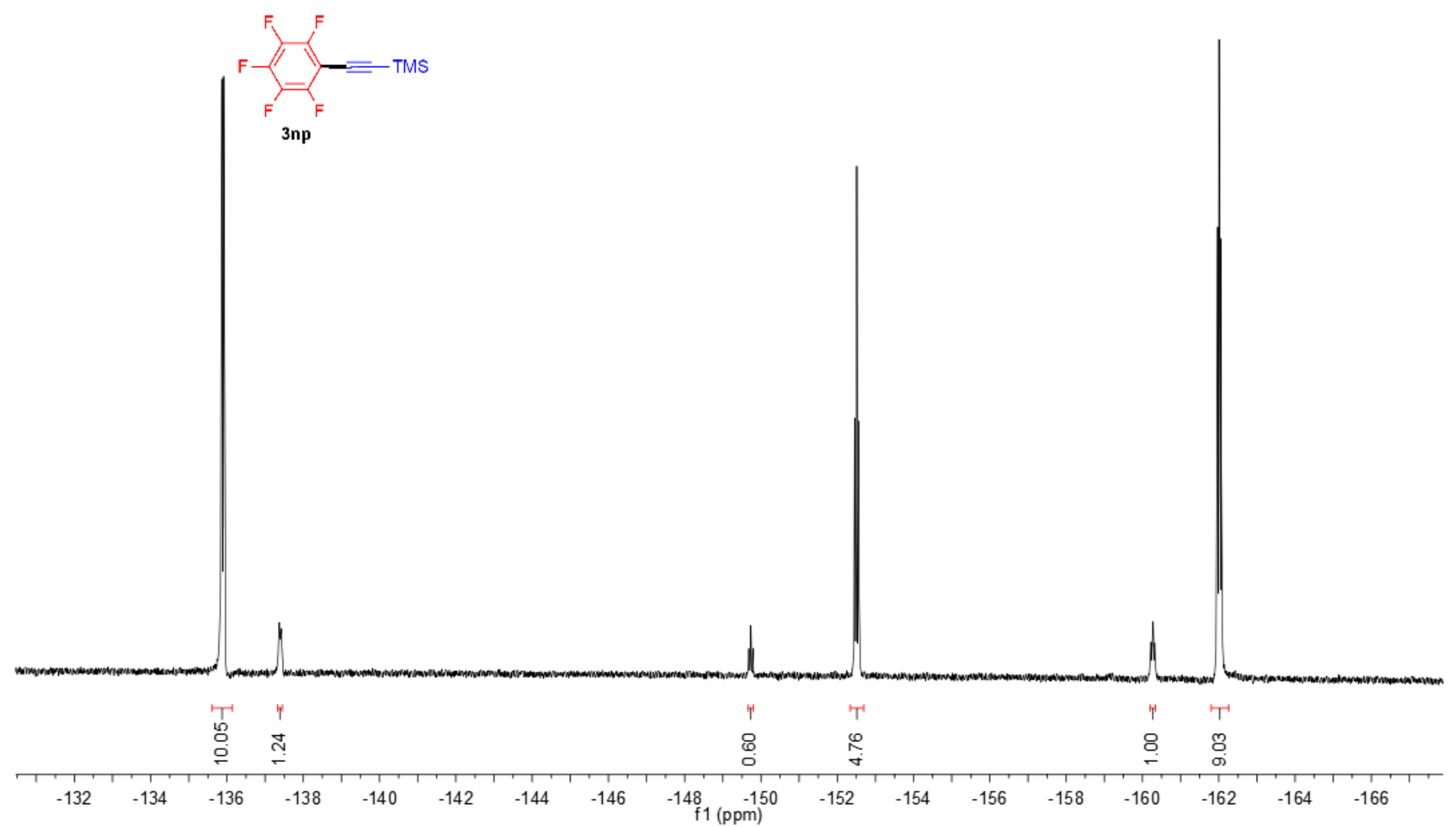

Note: The sample contains $\sim 10 \%$ inseparable impurity of 3np' that results from the further alkynylation of $\mathbf{3 n p}$. 


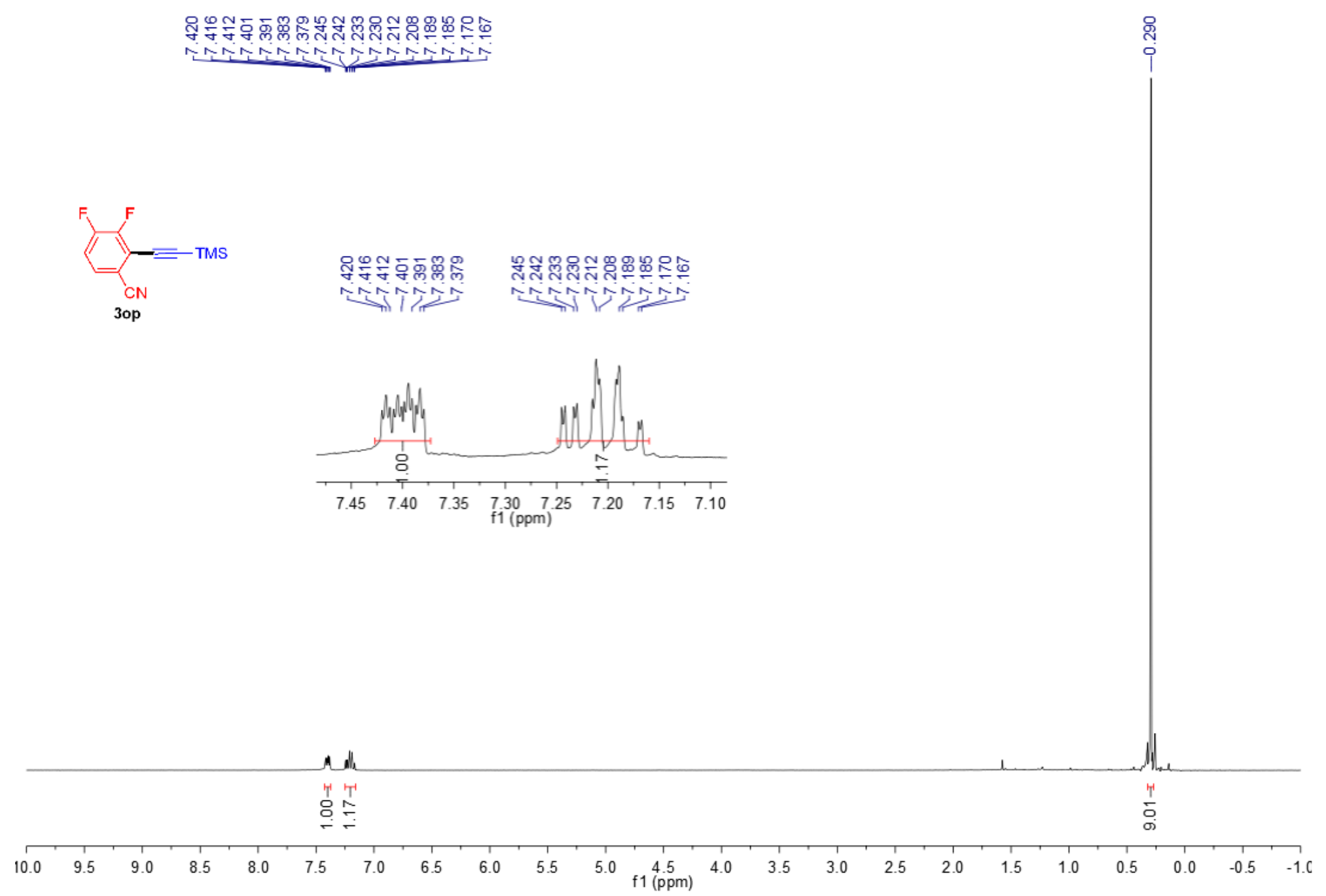




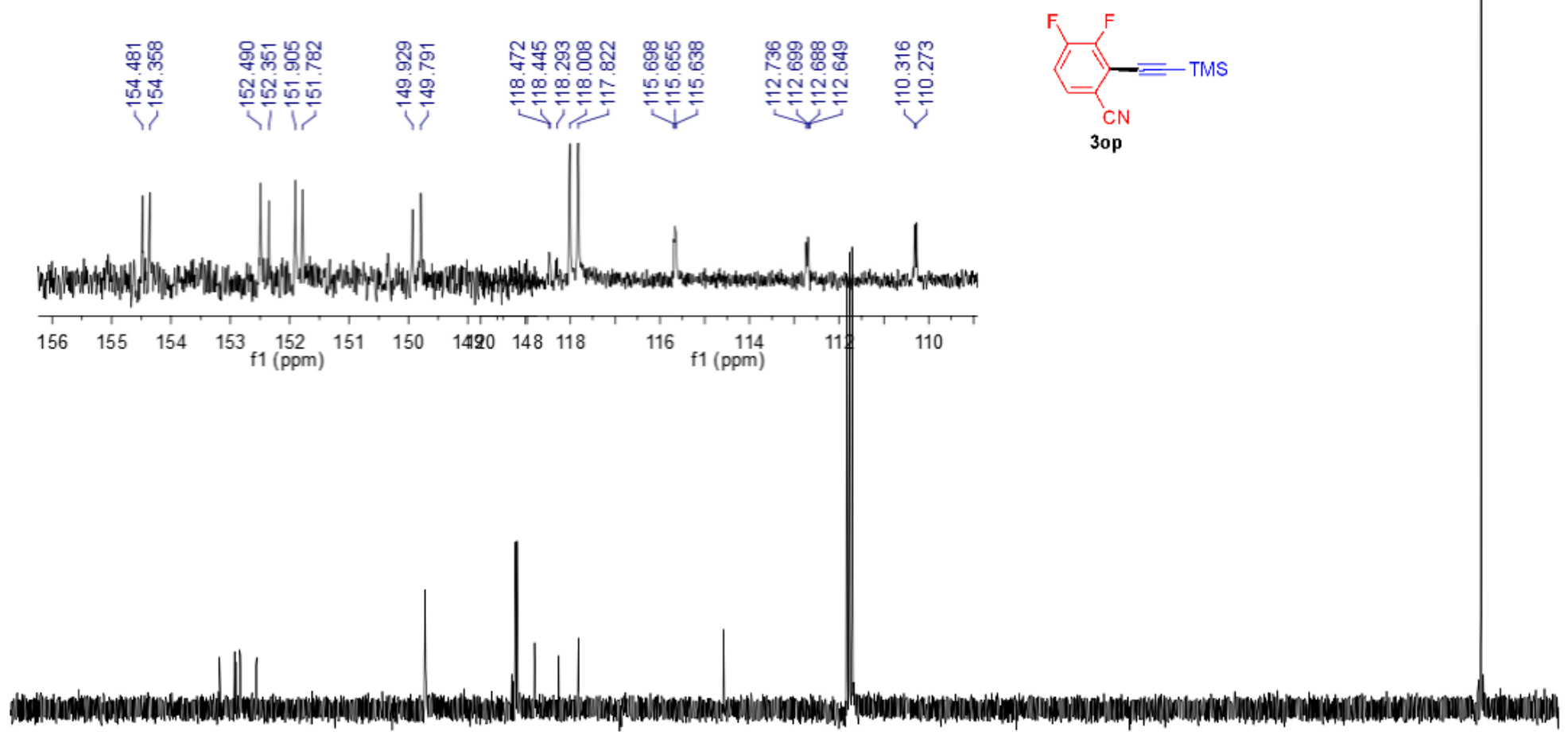

180

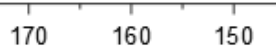

$150 \quad 140$ 


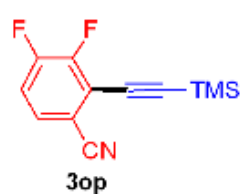

3op

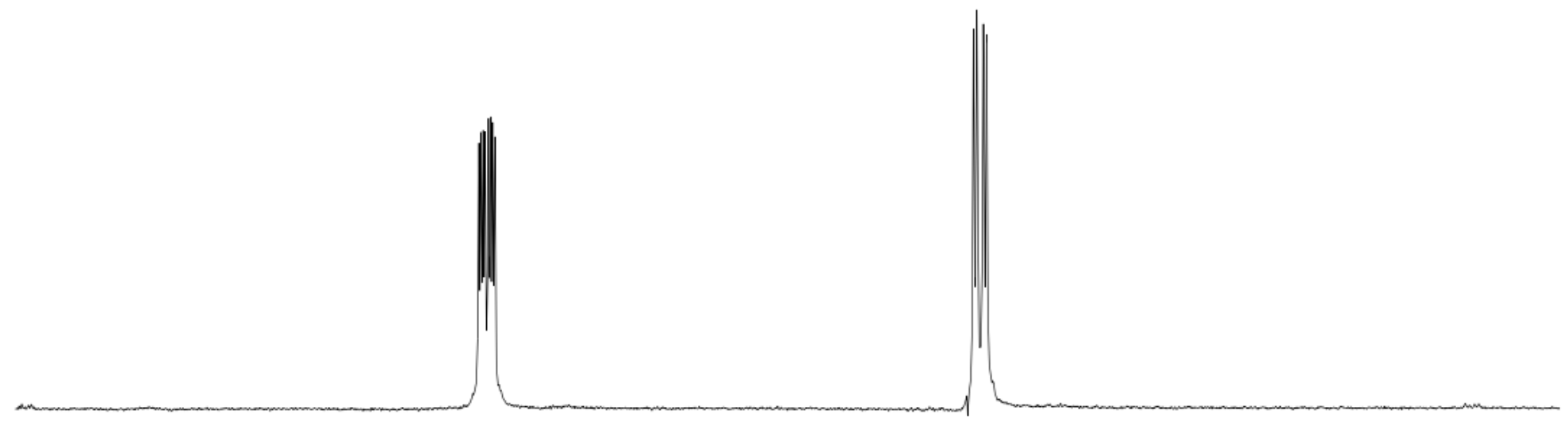

$\begin{array}{llll}124.5 & -125.0 & -125.5 & -126.0\end{array}$ 


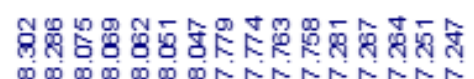

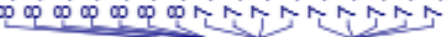
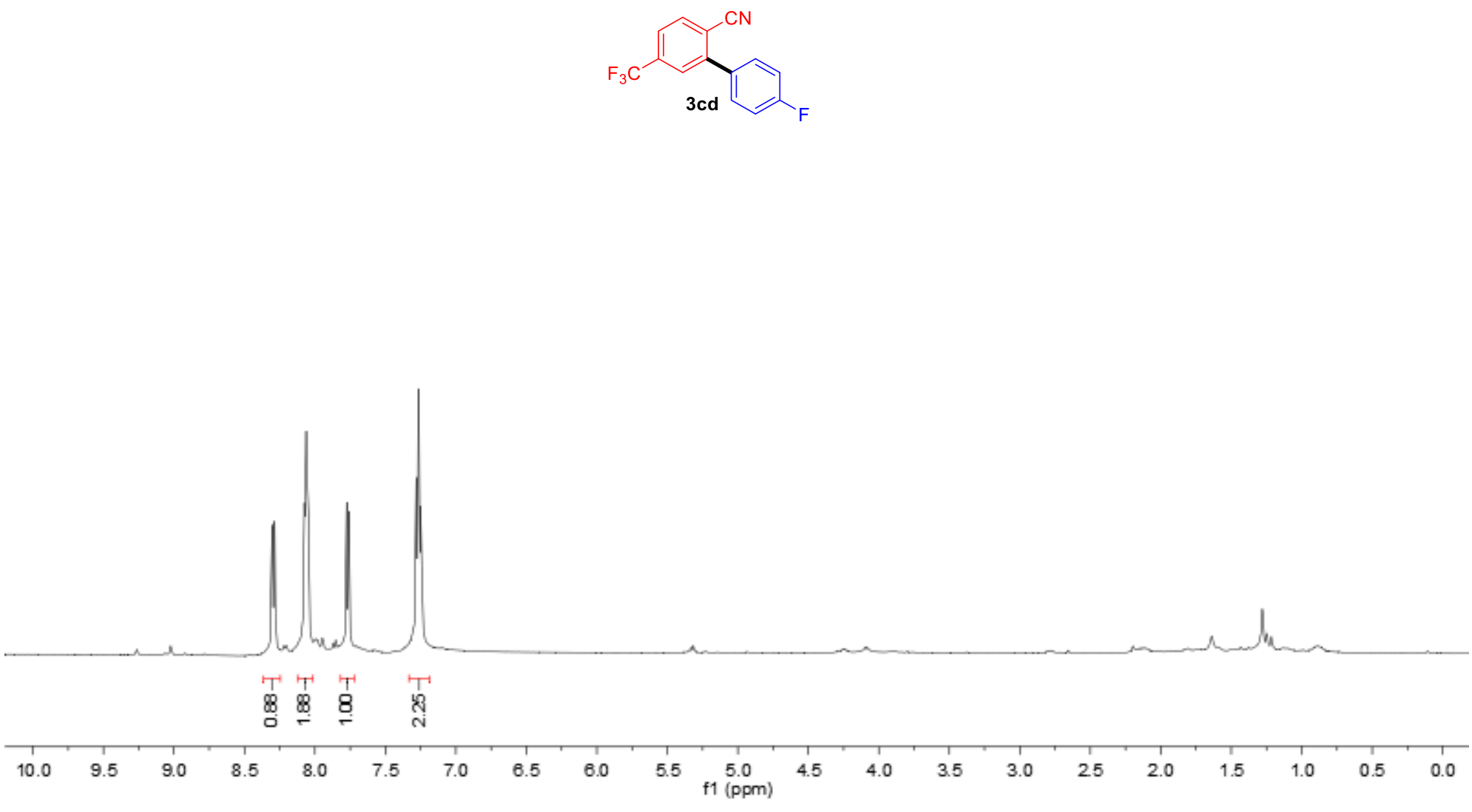

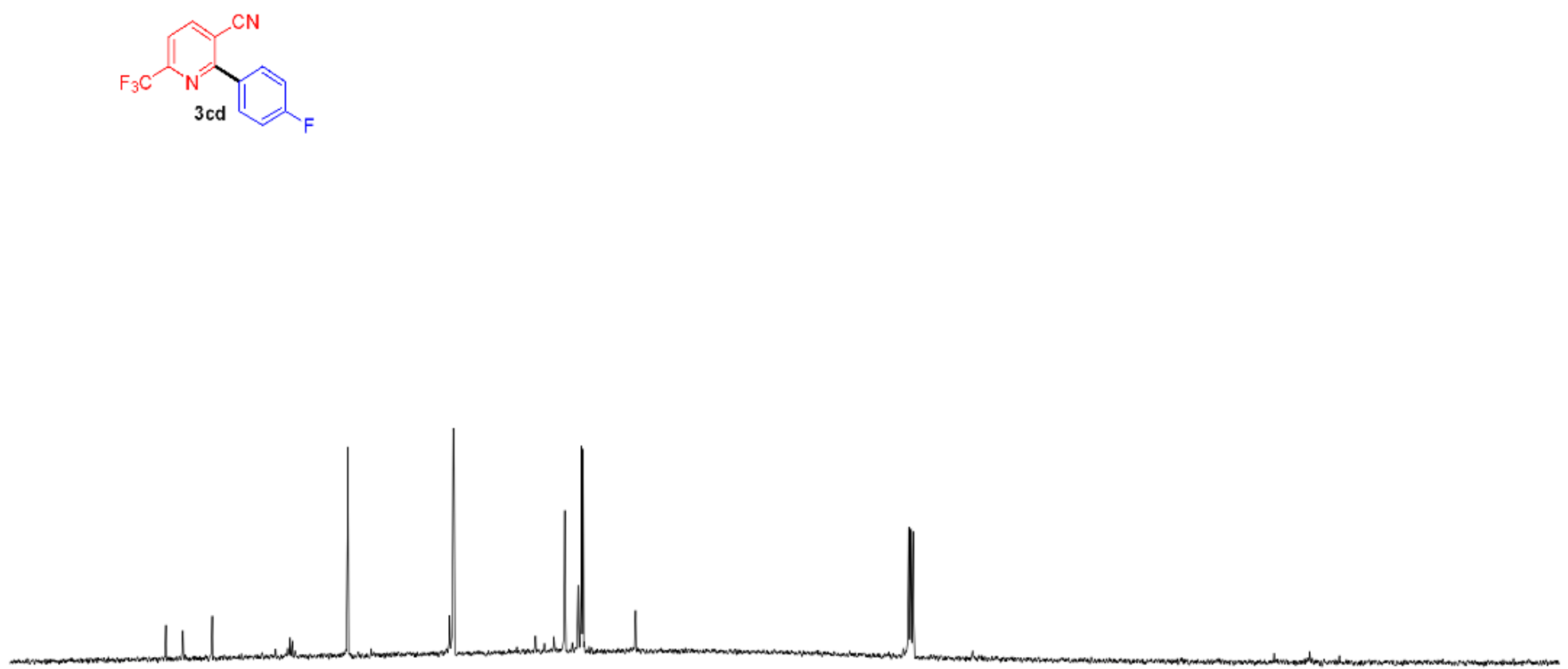
$\underset{\substack{0 \\ 0}}{\substack{1 \\ i}}$

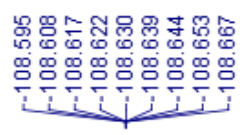

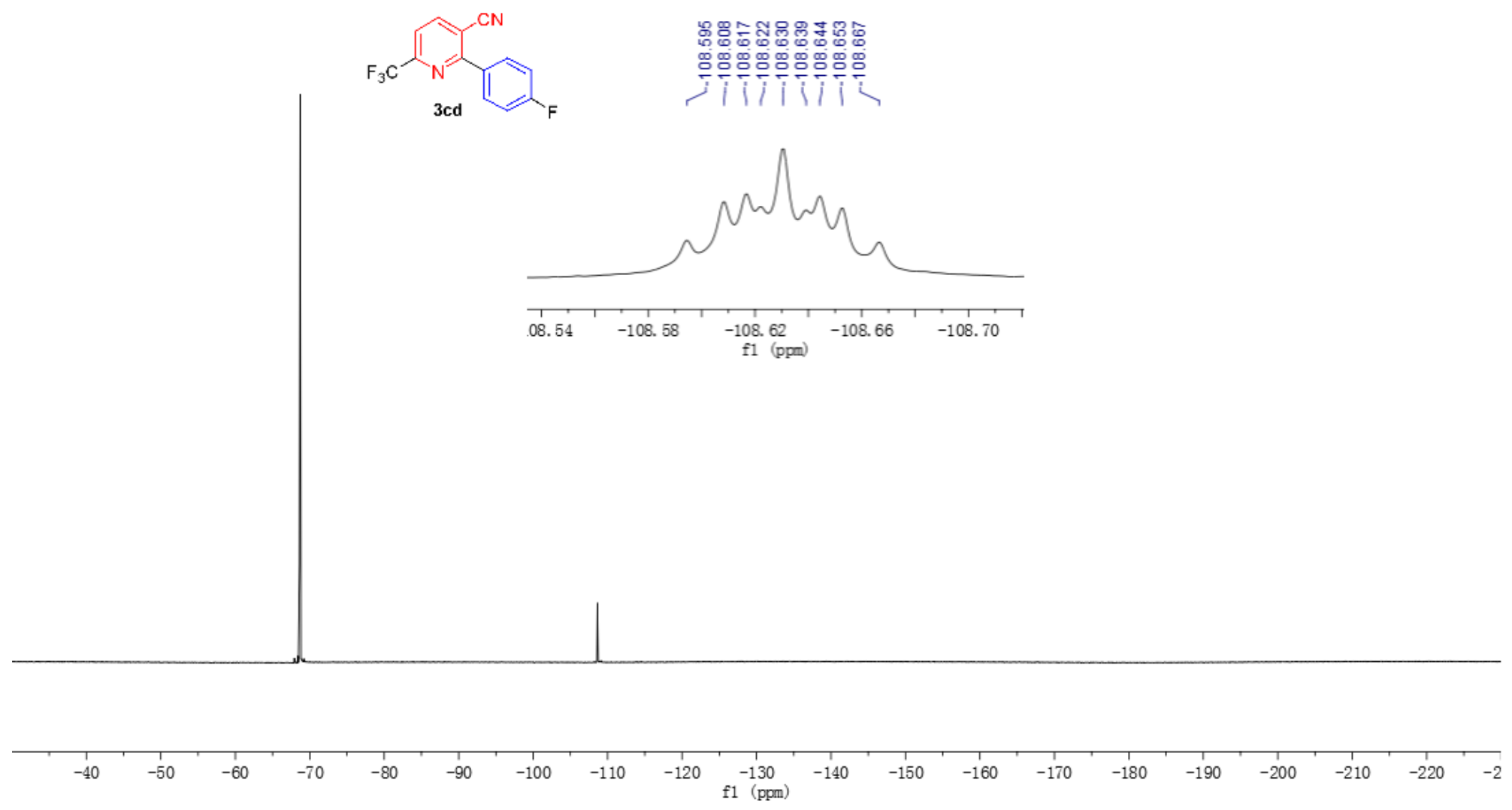

S86 


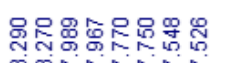

on

(c)

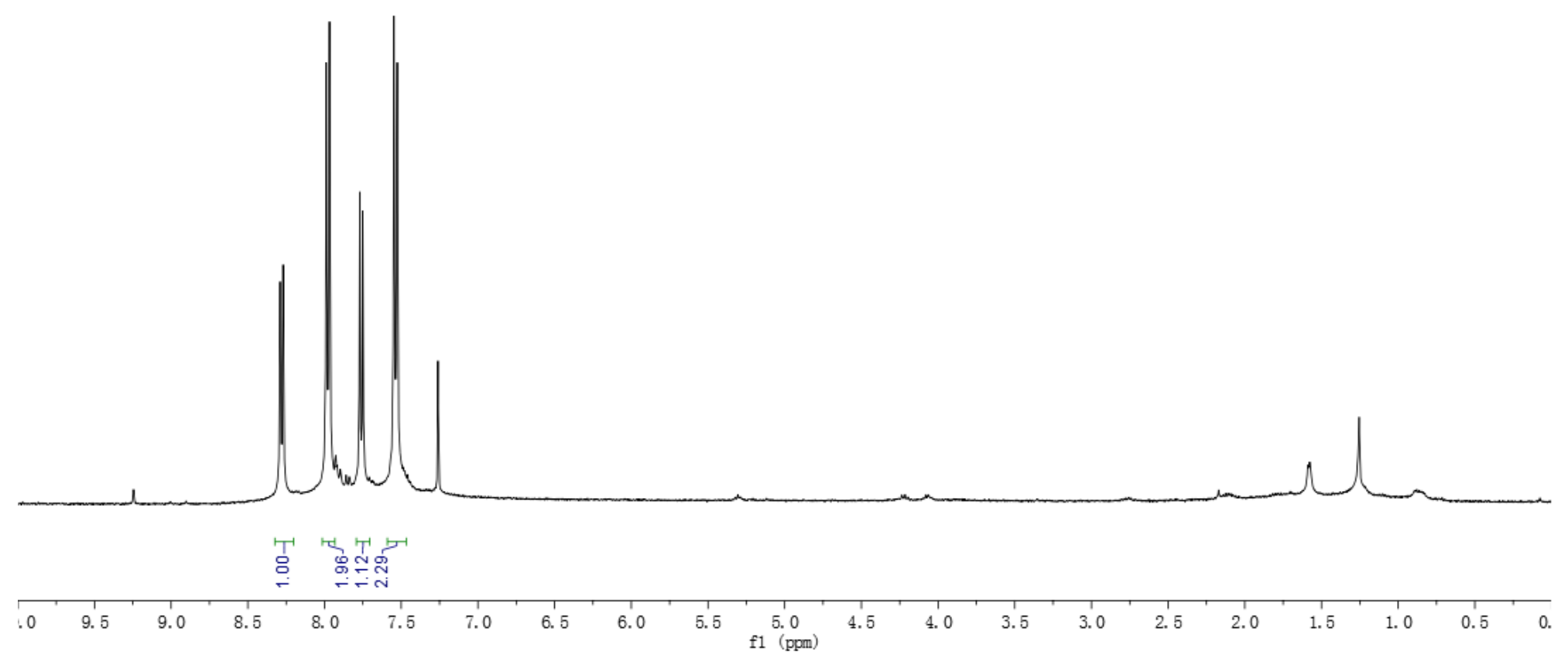




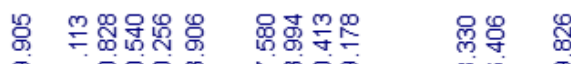

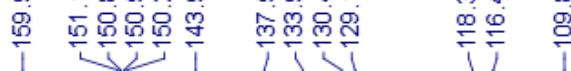

กำ

赫
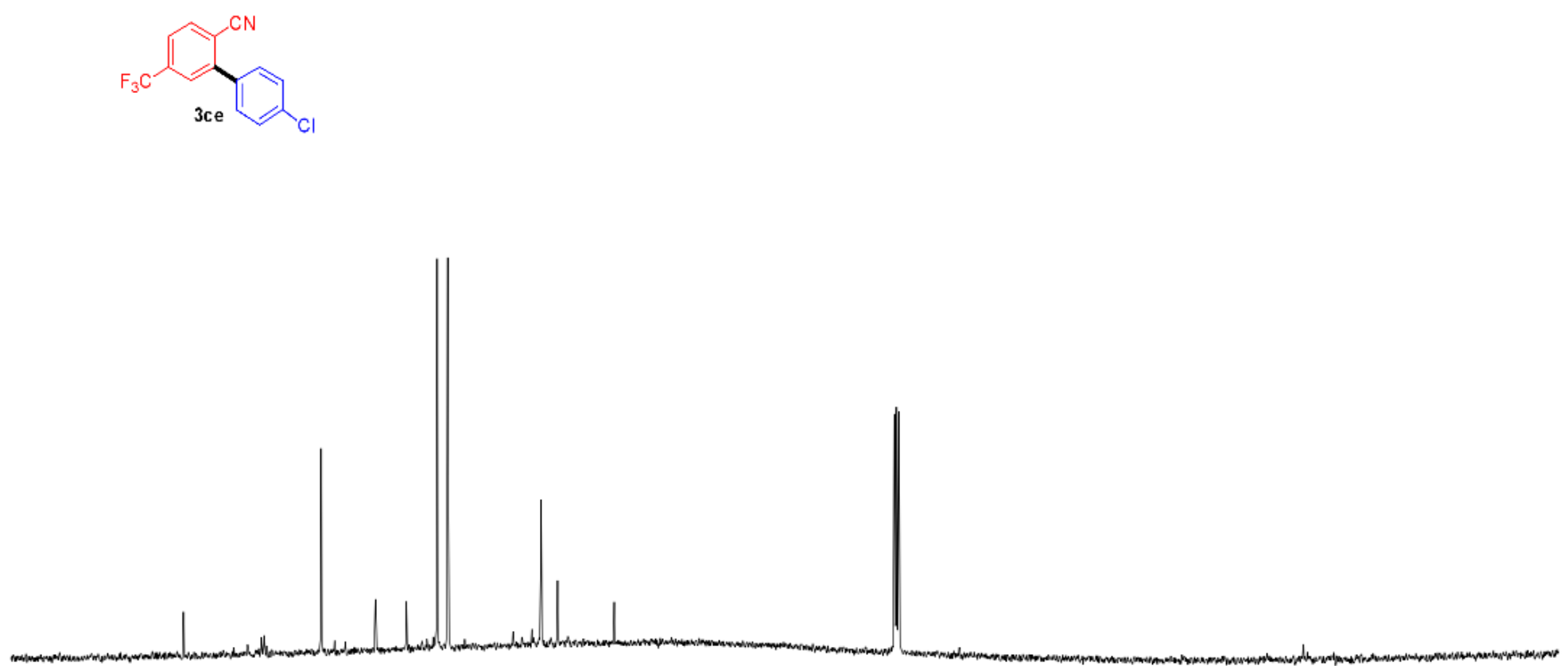


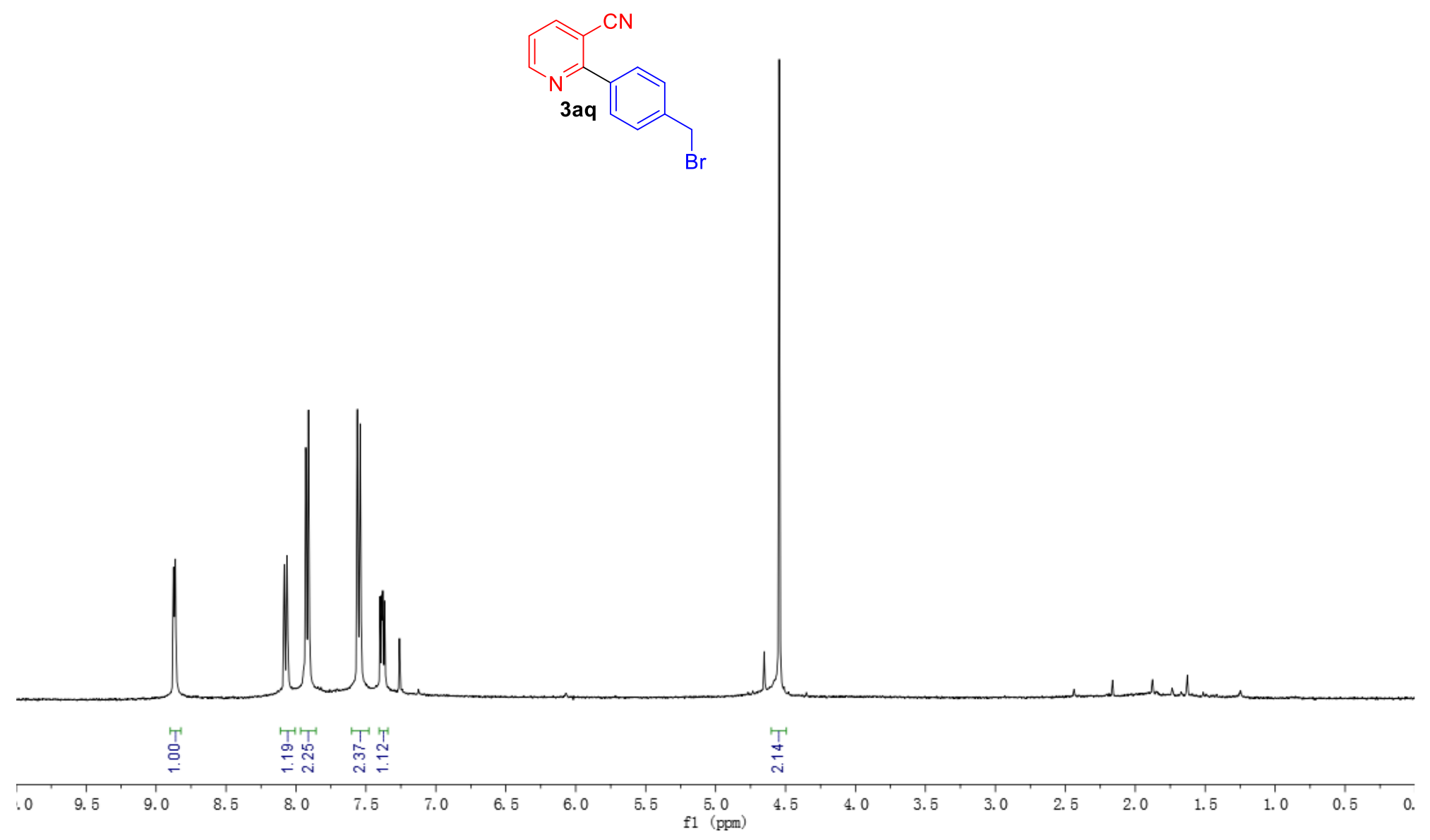



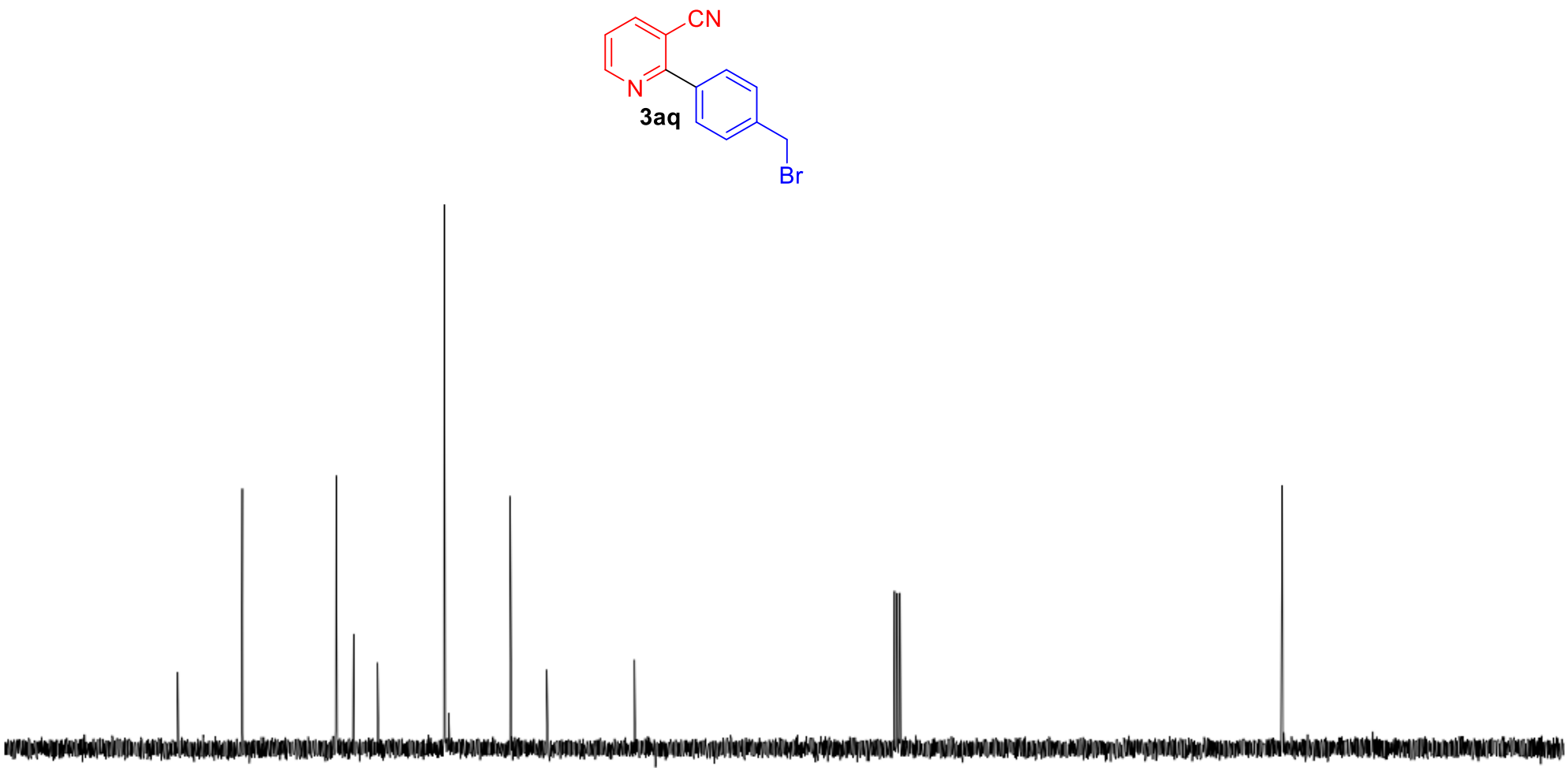

$\begin{array}{llll}180 & 170 \quad 160 & 150\end{array}$

130

120

$10 \quad 100$

$\stackrel{90}{\mathrm{f} 1}(\mathrm{ppm})$

$80 \quad 70$

60

$40 \quad 30$ $20 \quad 10$ 

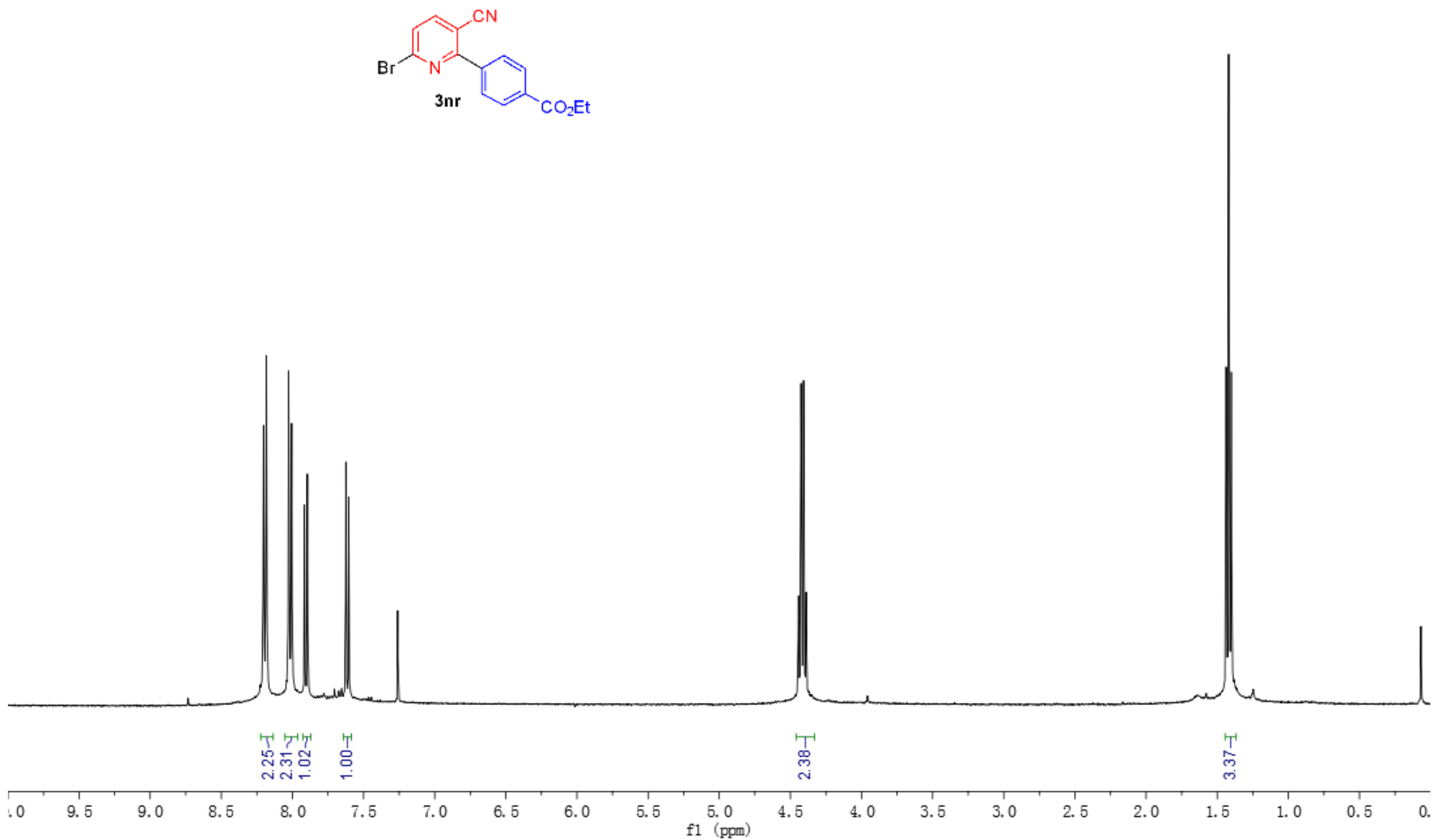


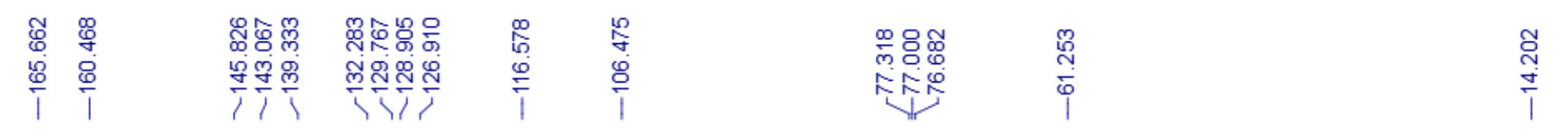

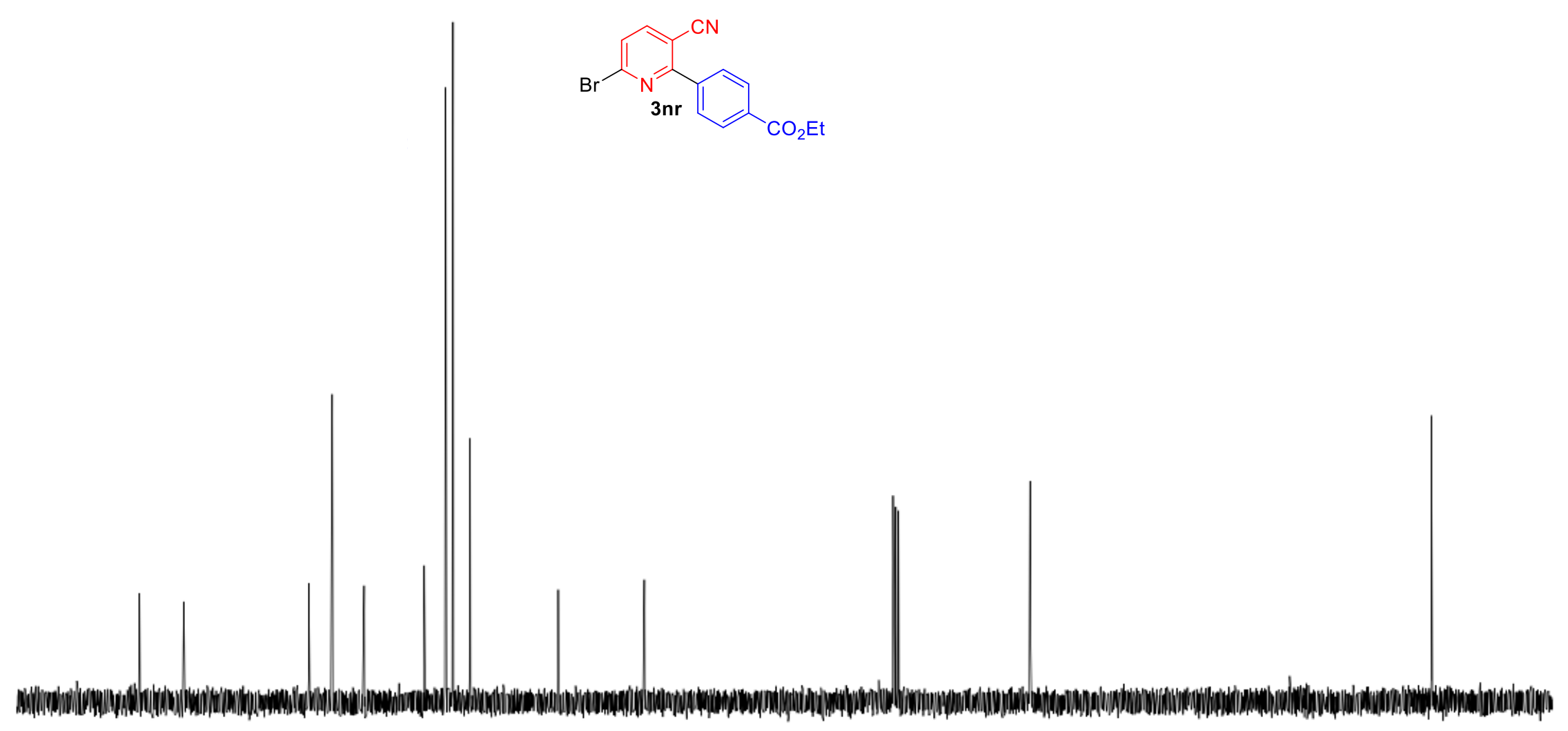

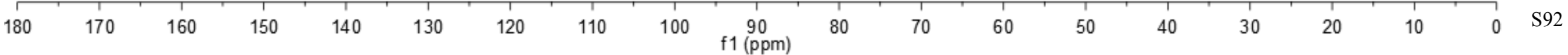




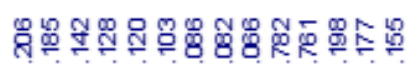

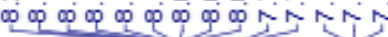

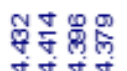

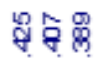

过

广

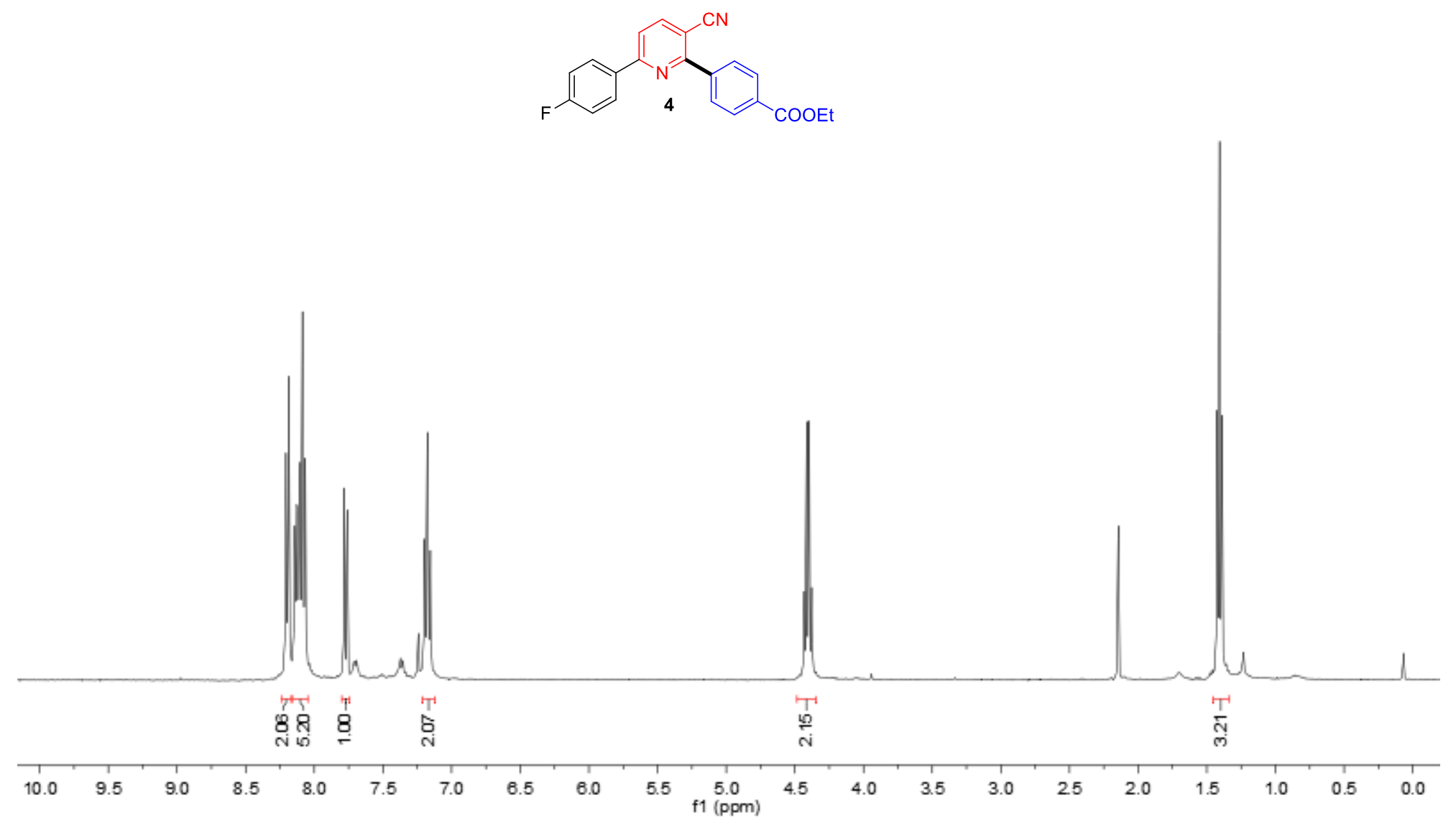




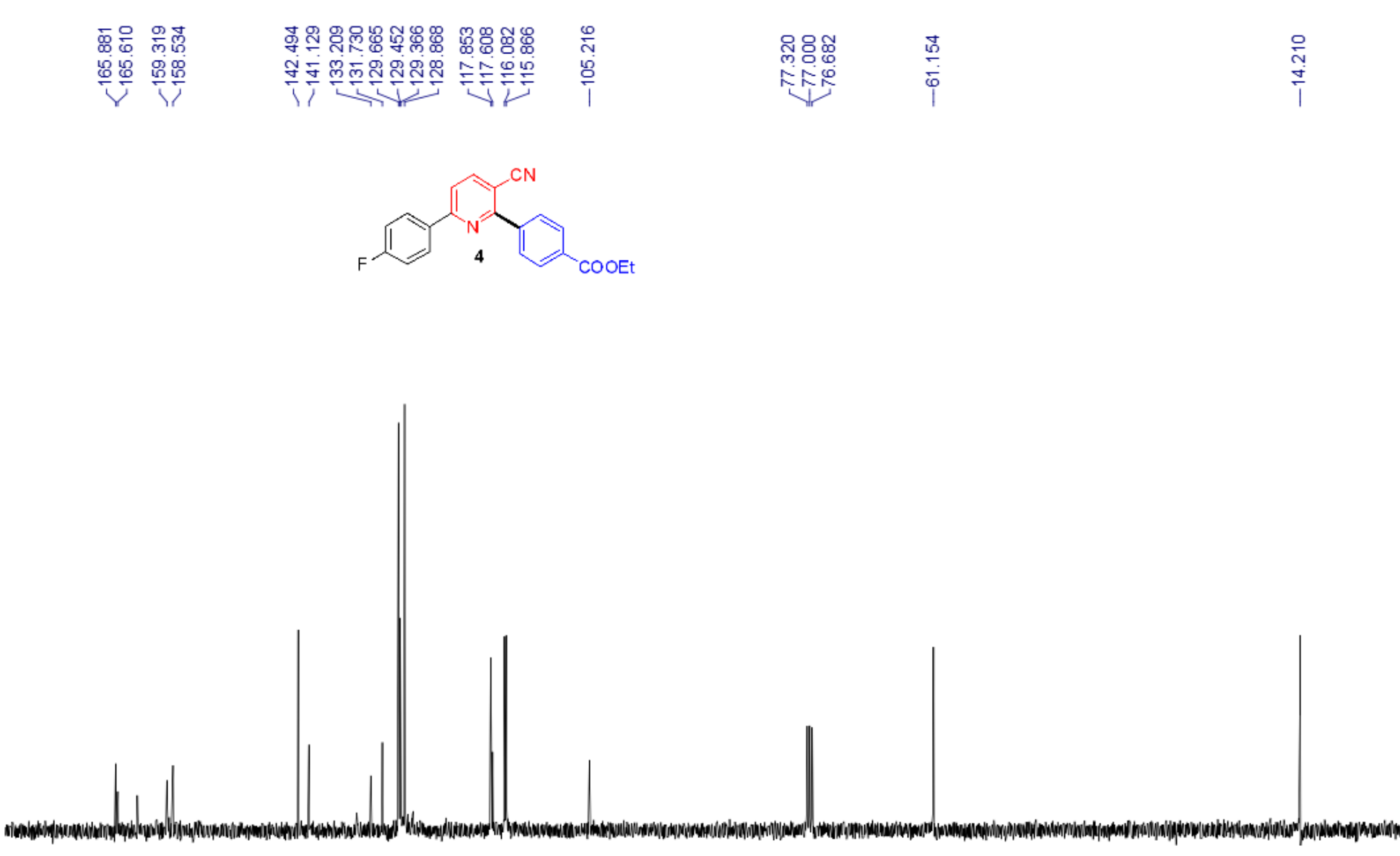




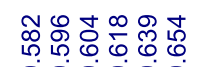

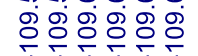

iniv
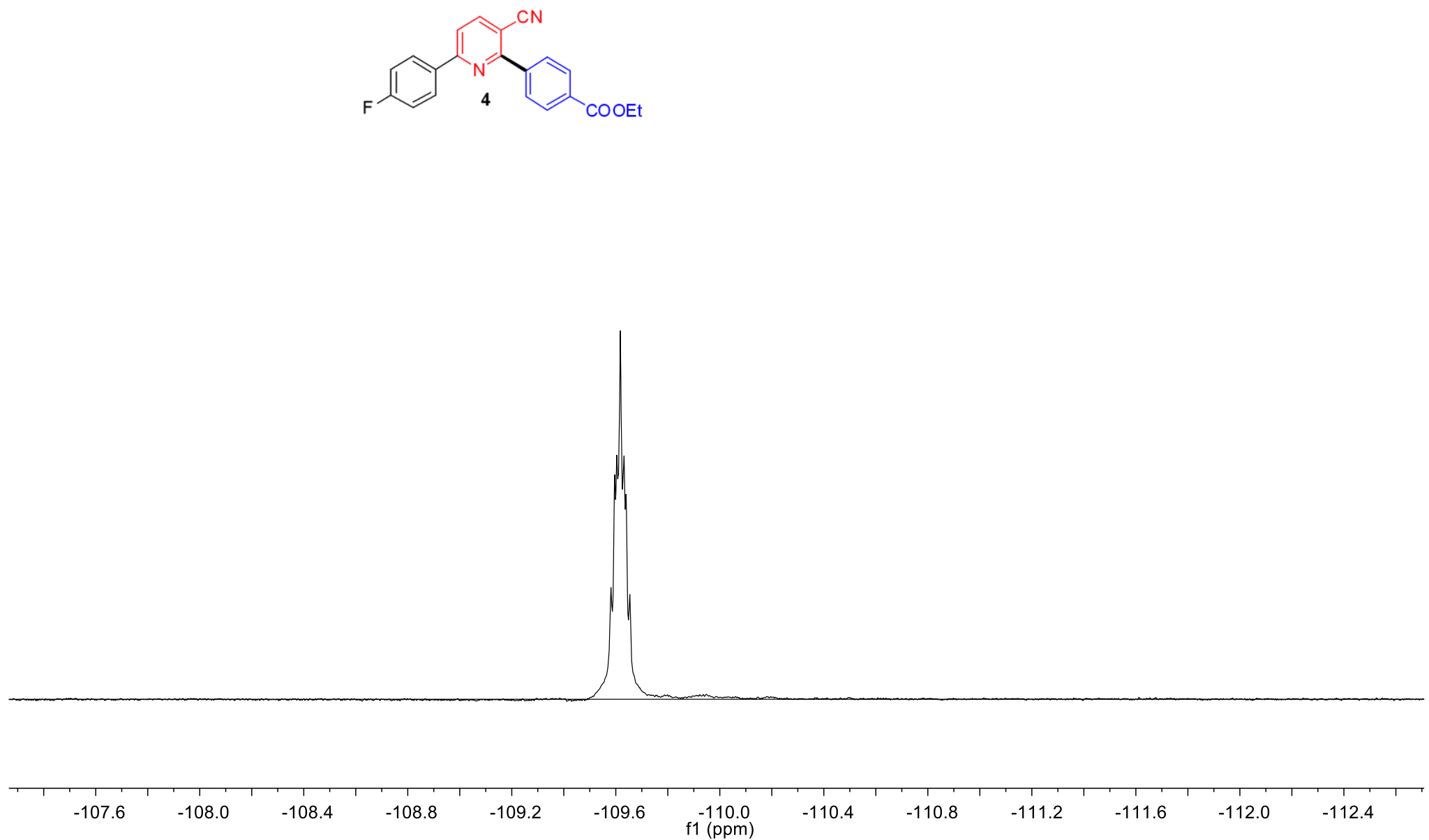


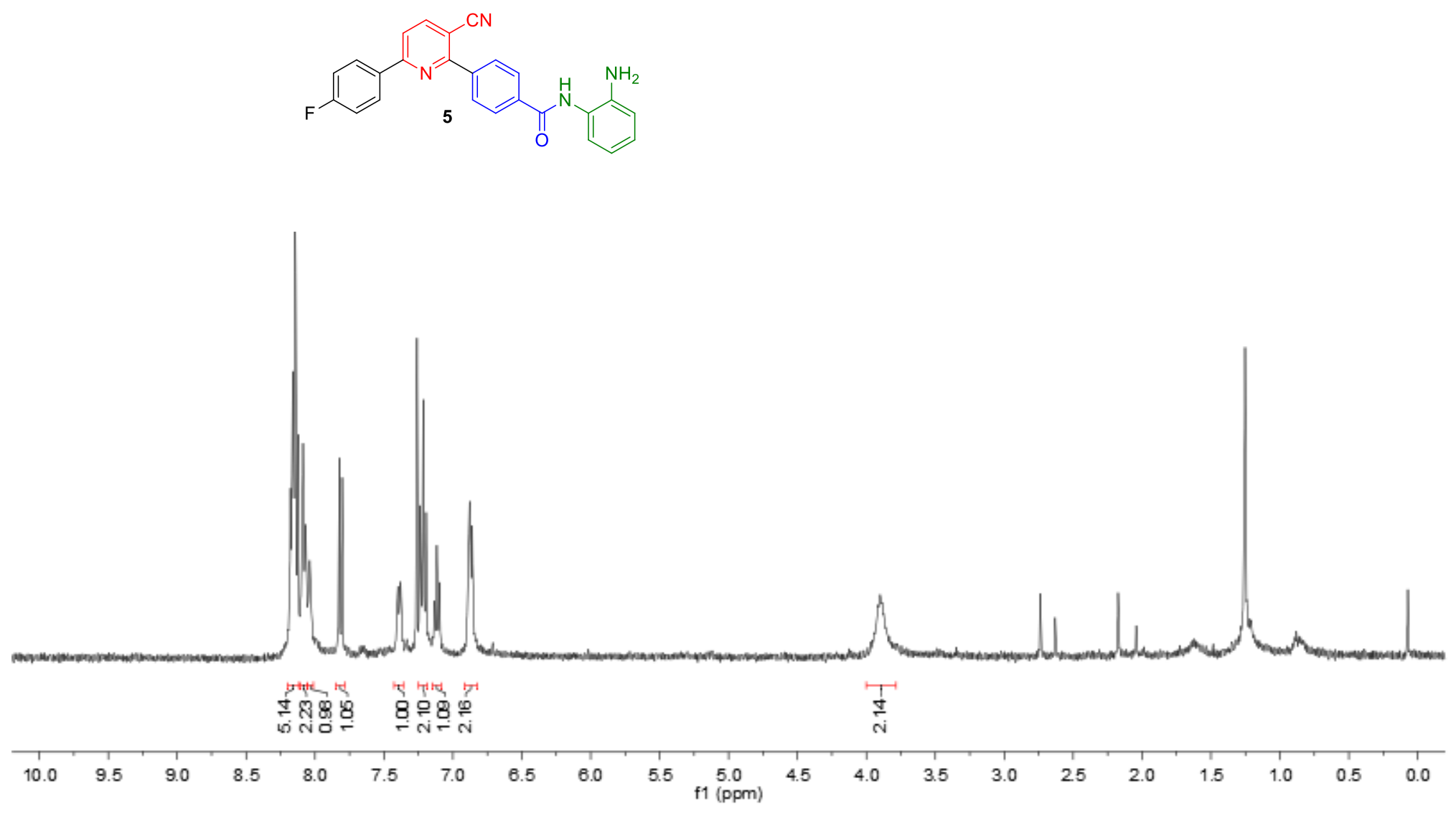




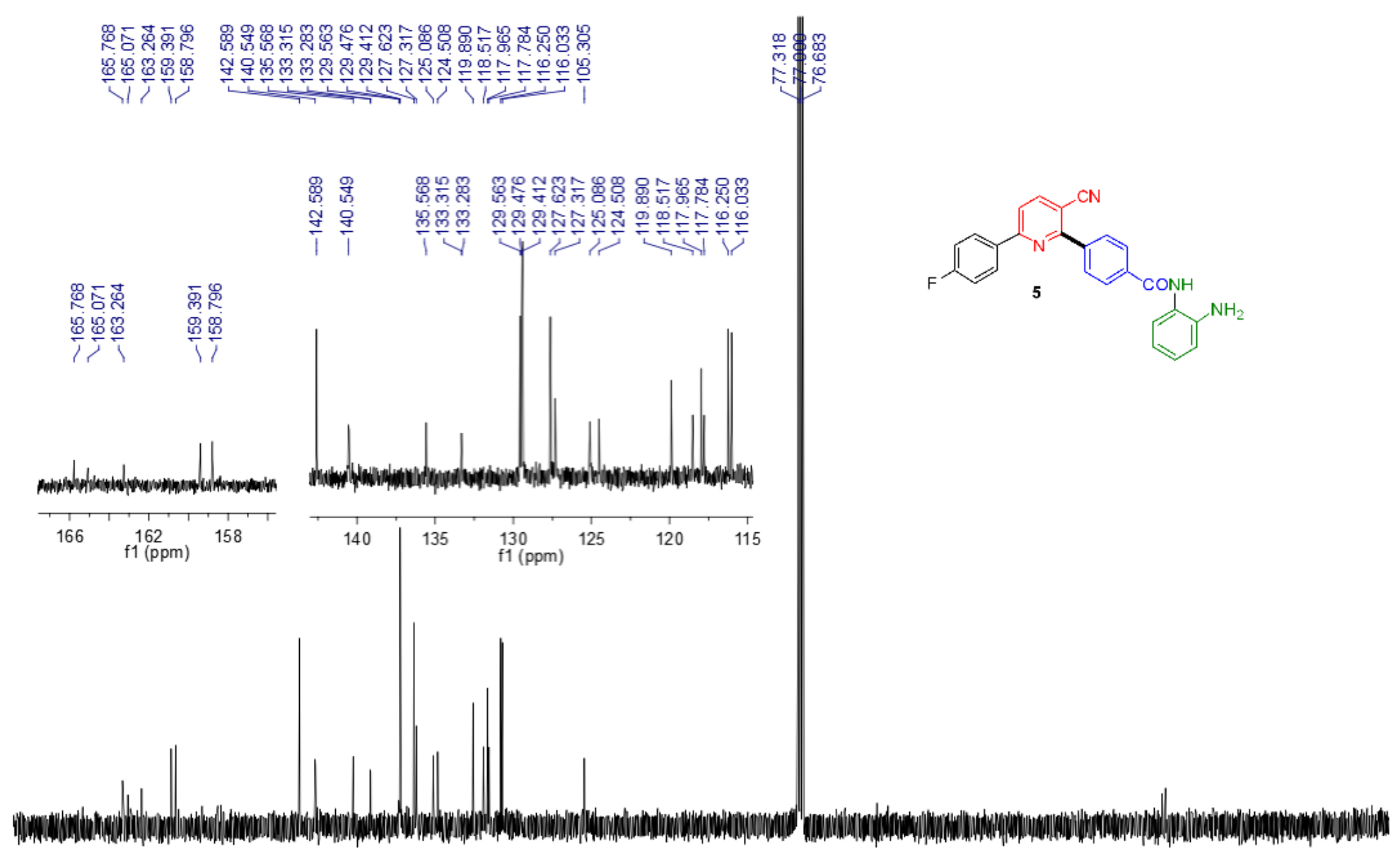

180 